ISSN 2090-3359 (Print)

ISSN 2090-3367 (Online)

\title{
$\mathbf{A} \mathbf{\Delta} \boldsymbol{\Sigma}$
}

\section{Advances in Decision Sciences}

\author{
Volume 25 \\ Issue 1 \\ March 2021
}

\author{
Michael McAleer \\ Editor-in-Chief \\ University Chair Professor \\ Asia University, Taiwan
}

Published by Asia University, Taiwan

ADS@ASIAUNIVERSITY 


\title{
A Critical Analysis of Some Recent Medical Research in Science on COVID-19*
}

\author{
Michael McAleer ** \\ Department of Finance, College of Management \\ and \\ Department of Bioinformatics and Medical Engineering \\ College of Information and Electrical Engineering \\ Asia University, Taiwan \\ and \\ Discipline of Business Analytics \\ University of Sydney Business School, Australia \\ and \\ Econometric Institute, Erasmus School of Economics \\ Erasmus University Rotterdam, The Netherlands \\ and \\ Department of Economic Analysis and ICAE \\ Complutense University of Madrid, Spain
}

Revised: June 2021

\footnotetext{
* For financial support, the author acknowledges the Australian Research Council and the Ministry of Science and Technology (MOST), Taiwan.

** Correspondence: michael.mcaleer@ gmail.com
} 


\begin{abstract}
The highly infectious and rapidly mutating SARS-CoV-2 virus that causes the COVID-19 disease is confronting the international community with few palatable options. The research output on COVID-19 has been revolutionary, especially in the medical and biomedical sciences, where the search for a vaccine is essential for the world to have a semblance of normality in a COVID19 normal world. Much of the advanced research has been distributed in the leading medical journals, where the latest medical research is distributed on a daily basis. The Science journals also have a new section on "Coronavirus: Research, Commentary, and News" that strives "to provide the best and most-timely research, analysis, and news coverage of COVID-19 and the coronavirus that causes it. All content is free to access." The purpose of this paper is to provide a critique of 144 interesting and highly topical research papers that have been published on general medicine, translational medicine, immunology, and robotics in Science. The diverse topics include the effectiveness of vaccines against mutations, establishing trust is hard work, distrust is easy, transmission of COVID-19 between mink and humans, the war against COVID-19 deniers, SARSCoV-2 is already endemic, COVID-19 efficacy, safety is essential for any vaccine for SARS-CoV2, aerosol transmission of SARS-CoV-2, pre-existing immunity to COVID-19 de novo mutations, and frequency and duration of COVID-19 reinfection, SARS-CoV-2 transmission between mink and humans, further known unknowns about T cell immunity to COVID-19, modeling confusion to clarity in a COVID-19 world, determining serological correlates of COVID-19 severity, what came first, effective COVID-19 vaccines or trial ethics?, safety et al. requirements for COVID-19 vaccines, controlling transmission heterogeneities and controllability of SARS-CoV-2 and COVID-19, transmission dynamics for a seemingly never ending COVID-19 pandemic, susceptibility to and long hauler effects from severe COVID-19, attack rates of COVID-19 in the Brazilian Amazon, humoral immunity to severe COVID-19, phylogenetic analysis of COVID-19 superspreading events, seasonal flu versus seasonal COVID-19, controlling the spread of COVID19, antibody responses to severe COVID-19, direct and indirect COVID-19 effects on the heart, government interventions against COVID-19, COVID-19 vaccines should be celebrated with, by and for all, countering the division on COVID-19 and mutated strains, encompassing tests for COVID-19, positive messages give hope, even in a COVID-19 world, how safe, effective, durable, and affordable are warp speed COVID-19 vaccines?, correlations of severe COVID-19 viral
\end{abstract}


responses, known and unknown long haul effects from COVID-19, how should approved COVID19 vaccines be distributed globally?, anaphylactic reactions to Pfizer's COVID-19 vaccine, open access is excellent, but who should pay, especially during COVID-19?, fast spreading COVID-19 mutations, the reality of COVID-19 in Africa, how does COVID-19 affect cancer and its treatment in Africa?, duration of immune memory to COVID-19, COVID-19 mutations and vaccinations, COVID-19 lineage dynamics, mutations, and vaccines, COVID-19, immunity, and endemicity, and T cells, immune systems, aging hosts in a COVID-19 world, social robots assisting humans in the COVID-19 era, herd Immunity against COVID-19 through infection or vaccination, crossreactive immunity to zoonotic diseases like COVID-19, and multivalent nanobodies, neutralization, suppression of COVID-19 escape mutants, public debate about science is essential in a COVID19 world, scientific reporting on COVID-19 in China, circadian rhythms and social jet lag in COVID-19, COVID-19 social control measures should have been implemented years ago, tackling student mental healthcare, especially during COVID-19, modelling prioritized COVID-19 vaccine strategies, optimal vaccination strategies for COVID-19, COVID-19 spillovers between species, comovements in science and policy during COVID-19, mathematical and statistical models to guide informed COVID-19 responses, preclinical efficacy and targeting of SARS-CoV-2 and COVID-19, engineering potent antibodies against SARS-CoV-2 and COVID-19, viral mutants escaping antibodies for treating COVID-19, can China help to alleviate bilateral debt payments during COVID-19?, pricing allowances to mitigate carbon dioxide emissions during COVID-19, sequencing SARS-CoV-2 RNA genomes to mitigate COVID-19, unanswered questions about antiviral immunity to COVID-19, neutralization of antibodies against escaped mutants, adapting vaccines to escaped mutants, public health and social measures during COVID-19 vaccinations, interpreting the outcomes of COVID-19 tests, disinfection against COVID-19 and antimicrobial resistance, augmenting antiviral immune responses of vaccines for COVID-19, age demographics propelling higher waves of COVID-19, escaping antibodies, recurrent deletions, evading immunity from SARS-CoV-2 and COVID-19, escaped mutants and herd immunity, new vaccines for mild to severe symptoms from COVID-19, escaped mutants of SARS-CoV-2 and COVID-19, are vaccines safe, effective, and durable against escaped mutants?, ethnic diversity in clinical trials for better COVID-19 vaccines, contact tracing for COVID-19 helps everyone, Science metric initiatives, viral sequencing in detecting COVID-19 strains and variants, single-domain antibodies that neutralize replication of SARS-CoV-2 and COVID-19, tracing SARS-CoV-2 and COVID-19 
in the UK, COVID-19 penalty for academic mothers, a balanced life during COVID-19, lipopeptides, intranasal prophylaxis, SARS-CoV-2 transmission, and impaired exhaustion and severe COVID-19, would a universal COVID-19 vaccine be safe, effective, and durable?, main protease of SARS-CoV-2 antivirals inhibit replication, temporal duration from COVID-19 to preCOVID-19 normal, mentoring and supervision are more important than the research topic, especially during COVID-19, can mutations in genes confer antibiotic resistance?, connecting violent entertainment and aggression, vaccines are essential for herd immunity from COVID-19, COVID-19 vaccines should be available to everyone, especially healthcare workers, which COVID-19 vaccines are safe, effective, and durable?, building confidence to face a $\mathrm{PhD}$ committee, especially during COVID-19, academic openness and national security during COVID-19, antiviral activity against SARS-CoV-2, ignoring the status of retracted COVID-19 papers is academic fake news, vaccinating children and adolescents against COVID-19, inhibiting SARSCoV-2 by enhancing host cell infection, testing COVID-19 megatrial therapies and treatments, new scientific frontiers in the time of COVID-19, questionable data throw school openings during COVID-19 under a spotlight, supply chains and COVID-19 vaccines: The need for speed, research lessons from COVID-19, transmissibility and impact of the UK COVID-19 lineage, neutralizing the impact of the UK COVID-19 lineage, within-host diversity and transmission of COVID-19, partial immunity from COVID-19 vaccine dosing regimes, great challenges need great ideas, especially for COVID-19, hoarding COVID-19 vaccines is unethical, COVID-19 vaccination passports for international travel, naming COVID-19 strains, variants, and lineages, zoom meetings for mothers during COVID-19, research is supposed to be enjoyable, COVID-19 or no COVID-19, asymptomatic and presymptomatic COVID-19 cases, impaired immunity through evolving SARS-CoV-2 variants, how many mentors is optimal, especially during COVID-19?, why is trust in COVID-19 vaccines volatile?, the science behind the AstraZeneca COVID-19 vaccine, practical carbon capture in a COVID-19 world, redesigning vaccines to be effective against COVID-19 variants, is it sensible to restrict AstraZeneca by age?, protecting scientists from COVID-19 burnout, adding technology to the NSF for scientific competitiveness, causality between COVID-19 cases and vaccination, expanding the narrative behind the development of COVID-19 vaccines, and confirmed cases, deaths, and vaccinations against COVID-19 in Brazil. 
As numerous mutations of the original wild-type SARS-CoV-2 coronavirus are still being detected, eternal vigilance is essential as the increasingly higher waves show no evidence of slowing down.

Keywords: Vaccines, safety, efficacy, mutations, transmission, deniers, endemic, aerosols, immunity, reinfections, translational medicine, immunology, robotics.

JEL: I11, I12, I13, I18. 


\section{Introduction}

The highly infectious and rapidly mutating SARS-CoV-2 virus that causes the COVID-19 disease is confronting the international community with few palatable options. The research output on COVID-19 has been revolutionary, especially in the medical and biomedical sciences, where the search for a vaccine is essential for the world to have a semblance of normality in a COVID19 normal world. Much of the advanced research has been distributed in the leading medical journals, where the latest medical research is distributed on a daily basis.

The Science journal, which is published by the American Association for the Advancement of Science (AAAS), has a new eLetters section on "Coronavirus: Research, Commentary, and News" that strives "to provide the best and most timely research, analysis, and news coverage of COVID19 and the coronavirus that causes it. All content is free to access." eLetters can also be submitted to "Letters to the Editor".

A statement from the editorial staff of Science that "Your eLetter, if accepted, should be viewable within a few days", the number of published eLetters would seem to provide testament, if any were needed, that it is difficult to publish in the journal. It would seem that Science does not send acceptance or rejection messages, although Science Immunology, Science Translational Medicine, and Science Robotics, seem to send acceptances, possibly because they receive fewer eLetters submissions. One can check on a paper several days after submission, which might include the message: "No eLetters have been published for this article."

The purpose of this paper is to supplement the existing reviews and perspectives by providing a critical analysis of 144 interesting and highly topical research papers that have been published on general medicine, translational medicine, immunology, and robotics in Science in recent months. Some associated eLetters have been published, as can be seen in the list of references.

Each of the papers evaluated here is worth highlighting as they cover several highly topical medical issues in the COVID-19 era, including the effectiveness of vaccines against mutations, establishing trust is hard work, distrust is easy, transmission of COVID-19 between mink and humans, the war against COVID-19 deniers, SARS-CoV-2 is already endemic, COVID-19 efficacy, safety is essential for any vaccine for SARS-CoV-2, aerosol transmission of SARS-CoV2, pre-existing immunity to COVID-19 de novo mutations, and frequency and duration of COVID19 reinfection, SARS-CoV-2 transmission between mink and humans, further known unknowns 
about T cell immunity to COVID-19, modeling confusion to clarity in a COVID-19 world, determining serological correlates of COVID-19 severity, what came first, effective COVID-19 vaccines or trial ethics?, safety et al. requirements for COVID-19 vaccines, controlling transmission heterogeneities and controllability of SARS-CoV-2 and COVID-19, transmission dynamics for a seemingly never ending COVID-19 pandemic, susceptibility to and long hauler effects from severe COVID-19, attack rates of COVID-19 in the Brazilian Amazon, humoral immunity to severe COVID-19, phylogenetic analysis of COVID-19 superspreading events, seasonal flu versus seasonal COVID-19, controlling the spread of COVID-19, antibody responses to severe COVID-19, direct and indirect COVID-19 effects on the heart, government interventions against COVID-19, COVID-19 vaccines should be celebrated with, by and for all, countering the division on COVID-19 and mutated strains, encompassing tests for COVID-19, positive messages give hope, even in a COVID-19 world, how safe, effective, durable, and affordable are warp speed COVID-19 vaccines?, correlations of severe COVID-19 viral responses, known and unknown long haul effects from COVID-19, how should approved COVID-19 vaccines be distributed globally?, anaphylactic reactions to Pfizer's COVID-19 vaccine, open access is excellent, but who should pay, especially during COVID-19?, fast spreading COVID-19 mutations, the reality of COVID19 in Africa, how does COVID-19 affect cancer and its treatment in Africa?, duration of immune memory to COVID-19, COVID-19 mutations and vaccinations, COVID-19 lineage dynamics, mutations, and vaccines, COVID-19, immunity, and endemicity, and T cells, immune systems, aging hosts in a COVID-19 world, social robots assisting humans in the COVID-19 era, herd immunity against COVID-19 through infection or vaccination, cross-reactive immunity to zoonotic diseases like COVID-19, and multivalent nanobodies, neutralization, suppression of COVID-19 escape mutants, public debate about science is essential in a COVID-19 world, scientific reporting on COVID-19 in China, circadian rhythms and social jet lag in COVID-19, COVID-19 social control measures should have been implemented years ago, tackling student mental healthcare, especially during COVID-19, modelling prioritized COVID-19 vaccine strategies, optimal vaccination strategies for COVID-19, COVID-19 spillovers between species, comovements in science and policy during COVID-19, mathematical and statistical models to guide informed COVID-19 responses, preclinical efficacy and targeting of SARS-CoV-2 and COVID-19, engineering potent antibodies against SARS-CoV-2 and COVID-19, viral mutants escaping antibodies for treating COVID-19, can China help to alleviate bilateral debt payments 
during COVID-19?, pricing allowances to mitigate carbon dioxide emissions during COVID-19, sequencing SARS-CoV-2 RNA genomes to mitigate COVID-19, unanswered questions about antiviral immunity to COVID-19, neutralization of antibodies against escaped mutants, adapting vaccines to escaped mutants, public health and social measures during COVID-19 vaccinations, interpreting the outcomes of COVID-19 tests, disinfection against COVID-19 and antimicrobial resistance, augmenting antiviral immune responses of vaccines for COVID-19, age demographics propelling higher waves of COVID-19, escaping antibodies, recurrent deletions, evading immunity from SARS-CoV-2 and COVID-19, escaped mutants and herd immunity, new vaccines for mild to severe symptoms from COVID-19, escaped mutants of SARS-CoV-2 and COVID-19, are vaccines safe, effective, and durable against escaped mutants?, ethnic diversity in clinical trials for better COVID-19 vaccines, contact tracing for COVID-19 helps everyone, Science metric initiatives, viral sequencing in detecting COVID-19 strains and variants, single-domain antibodies that neutralize replication of SARS-CoV-2 and COVID-19, tracing SARS-CoV-2 and COVID-19 in the UK, COVID-19 penalty for academic mothers, a balanced life during COVID-19, lipopeptides, intranasal prophylaxis, and SARS-CoV-2 transmission, and impaired exhaustion and severe COVID-19, would a universal COVID-19 vaccine be safe, effective, and durable?, main protease of SARS-CoV-2 antivirals inhibit replication, temporal duration from COVID-19 to preCOVID-19 normal, mentoring and supervision are more important than the research topic, especially during COVID-19, can mutations in genes confer antibiotic resistance?, connecting violent entertainment and aggression, vaccines are essential for herd immunity from COVID-19, COVID-19 vaccines should be available to everyone, especially healthcare workers, which COVID-19 vaccines are safe, effective, and durable?, building confidence to face a PhD committee, especially during COVID-19, academic openness and national security during COVID-19, antiviral activity against SARS-CoV-2, ignoring the status of retracted COVID-19 papers is academic fake news, vaccinating children and adolescents against COVID-19, inhibiting SARSCoV-2 by enhancing host cell infection, testing COVID-19 megatrial therapies and treatments, new scientific frontiers in the time of COVID-19, questionable data throw school openings during COVID-19 under a spotlight, supply chains and COVID-19 vaccines: The need for speed, research lessons from COVID-19, transmissibility and impact of the UK COVID-19 lineage, neutralizing the impact of the UK COVID-19 lineage, within-host diversity and transmission of COVID-19, partial immunity from COVID-19 vaccine dosing regimes, great challenges need great ideas, 
especially for COVID-19, hoarding COVID-19 vaccines is unethical, COVID-19 vaccination passports for international travel, naming COVID-19 strains, variants, and lineages, zoom meetings for mothers during COVID-19, research is supposed to be enjoyable, COVID-19 or no COVID-19, asymptomatic and presymptomatic COVID-19 cases, impaired immunity through evolving SARS-CoV-2 variants, how many mentors is optimal, especially during COVID-19?, why is trust in COVID-19 vaccines volatile?, the science behind the AstraZeneca COVID-19 vaccine, practical carbon capture in a COVID-19 world, redesigning vaccines to be effective against COVID-19 variants, is it sensible to restrict AstraZeneca by age?, protecting scientists from COVID-19 burnout, adding technology to the NSF for scientific competitiveness, causality between COVID-19 cases and vaccination, expanding the narrative behind the development of COVID-19 vaccines, and confirmed cases, deaths, and vaccinations against COVID-19 in Brazil.

As discussed in a number of research papers on COVID-19, many papers have been included in the World Health Organization's (2020) "WHO COVID-19 Global literature on coronavirus disease", which is intended to bring "the world's scientists and global health professionals together to accelerate the research and development process, and develop new norms and standards to contain the spread of the coronavirus pandemic and help care for those affected." The list currently has well over one million entries.

As numerous mutations of the original wild-type coronavirus are being detected, eternal vigilance is essential as the increasingly higher waves show no evidence of slowing down.

\section{Effectiveness of Vaccines Against Mutations}

The innovative research by Hou, Chiba, Halfmann, Ehre, Kuroda, Dinnon III et al. (2020) finds, among others, that:

"Some sera and mAbs, such as serum \#1 and REGN10987, displayed slightly different neutralization potencies against the two viruses, suggesting subtle differences in the Ab binding properties. As a limitation, the virus genotype in most serum donors remains unknown. These data also suggest that the current vaccine approaches directed against WT spike should be effective against the D614G strains." 
As an interpretation, the third sentence regarding effectiveness of vaccines does not seem to follow from the previous two, namely subtle differences in binding properties and limitations regarding unknown serum donors.

It also seems questionable that better binding necessarily improves vaccine effectiveness, in light of the unknown replication rates of mutations.

\section{Establishing Trust Is Hard Work, Distrust is Easy}

The prescient and convincing editorial by Tuckson (2020) raises a number of challenges. Distrust arises because of a lack of confidence on any issue, be it healthcare, income inequality, socio-economic disparities, and/or racial and ethnic inequalities, among others.

The question arises as to what the medical, scientific and healthcare professions have done to educate the broader community about the need for drastic change, especially during the COVID19 pandemic, which has heightened awareness about the continuing and expanding problems.

Fighting ignorance is difficult at the best of times, but what is required is greater vigilance, and responding with scientific and accurate information in a world of fake news and growing distrust.

\section{Transmission of COVID-19 Between Mink and Humans and Mass Culling}

Though unfortunate for the destroyed mink and the ongoing research into transmission of the SARS-CoV-2 virus and COVID-29 disease between mink and humans (see Lesté-Lasserre, 2020)), it would be useful to investigate the mutation rates from mink to mink, from humans to humans, from mink to humans, and from humans to mink, as a guide to the safety and efficacy of COVID19 vaccines.

If the replication rates of mutations were known, as well as their binding properties, this information could lead to greater vaccine effectiveness.

Despite a mass culling of millions of mink in Denmark, COVID-19 has spread to mink farms in Poland (as of 23 November 2020).

\section{The War Against COVID-19 Deniers}


The War against COVID-19 is being won by COVID-19 deniers, with similar sad outcomes for the War on Anything, including Ignorance, Income Inequality, Sickness, Poverty, Human Trafficking, Domestic Violence, Child Abuse, and Reduced Life Expectancy, among many others.

Eternal vigilance in any war is essential, especially in the news media and social media, otherwise the COVID-19 deniers, ignorant, insensitive, abusers, flat earthers, anti-maskers, antilockdowns, and many other uninformed critics of medical science and research, will win the day.

As discussed in Bharti (2020), Society needs scientists and medical experts to exercise their loud voices in the public domain to direct the COVID-19 narrative and protect all individuals, especially those who need it most.

\section{SARS-CoV-2 is Already Endemic}

The informative research on SARS-CoV-2 by Shaman and Galanti (2020) is most welcome for specialists and non-specialists alike.

The long-term consequences of long haulers, namely those who have been infected with the virus, and have purportedly recovered from the external symptoms, aka asymptotic carriers, have some or all of the following long-terms effects, among others:

(1) unknown duration of protective immunity;

(2) unknown duration of waning immunity and immune escape;

(3) unknown number and severity of mutations;

(4) unknown seasonal variations in COVID-19;

(5) possibility of reinfection with a mutated virus;

(6) loss of taste and smell;

(7) loss of hearing;

(8) respiratory distress;

(9) greater likelihood of mental health problems;

higher onset of Parkinson's disease.

\section{COVID-19 Efficacy}


The world anxiously awaits a safe, effective, affordable, and widely distributed vaccine that will provide direct and indirect short-term and long-term protection against the SARS-CoV-2 virus that causes COVID-19.

The detailed and prescient research findings by Lipsitch and Dean (2020) should be of great interest to medical and healthcare workers, and also to those who are not medically proficient.

Several vaccines undergoing clinical laboratory trials in the USA and other countries for the SAS-CoV-2 virus that causes the COVID-19 disease do not seem to have been clinically tested on the young (under 18), on pregnant women, on the aged (over 70), on the presently sick, on any patient with pre-existing conditions and comorbidities, on individuals taking recreational or hard drugs, on the poor, and/or on racial and ethnic minorities, among many others

It is government mandated in the USA that prisoners in correctional facilities, where COVID19 is widespread, are not able to give "informed consent" to be included in clinical trials while incarcerated.

That does not sound like a representative sample of any known general population worldwide.

\section{Safety is Essential for Any Vaccine for SARS-CoV-2}

A safe, effective, affordable, durable, and widely distributed vaccine that will provide extended protection against the SARS-CoV-2 virus that causes COVID-19 will not be a panacea, but should lead to mitigation of the pandemic.

The emphasis of any media announcement is typically on a safe and effective vaccine that satisfies the protocols that will enable wide distribution and deployment throughout the community.

The detailed and informative research findings on vaccine safety by Knipe, Levy, Fitzgerald and Mühlberger (2020) are welcome to medical specialists and every member of society as a safe and effective vaccine is essential for the world to survive in a COVID-19 normal world.

Several vaccines are being developed around the world, with some developers broadcasting an effective rate of around $95 \%$, which might be compared with the effective rate of around $80 \%$ for the seasonal flu vaccine that has been around since the mid-1940s.

The SARS-CoV-2 virus is far more infectious and has higher and faster rates of mutation than does the seasonal flu. 
The virus has a short incubation period in order for an infected individual to infect others who are exposed.

Subsequently, this leads to a short generation of infected individuals to infect other individuals who are exposed.

It is open to question whether clinical laboratory trials are evaluating safety and effectiveness of a vaccine on the original wild-type coronavirus, which will not necessarily be safe or effective against the numerous mutations and strains that have been discovered since its inception.

\section{Aerosol Transmission of SARS-CoV-2}

Transmission of the SARS-CoV-2 virus that causes COVID-19 should be of immense concern to every healthcare worker who relies on PPE as that might not be safe and effective in the face of airborne transmissions in both confined and open spaces (see Prather, Marr, Schooley, McDiarmid, Wilson and Milton (2020)).

Comprehensive discussions of the transmission of COVID-19 by droplets and the lighter evaporating aerosols are presented in Jayaweera et al. (2020) and Klompas, Baker and Rhee (2020).

As shown in Zeng, Wang, Li et al. (2020), the SARS-CoV-2 virus can be carried by aerosols that remain suspended in the air and carried by currents, so that social distancing norms will be ineffective in preventing infection.

In short, medical masks, face shields, social distancing, and the wearing of eyeglasses might not provide adequate protection against infection by the SARS-CoV-2 virus.

\section{Pre-existing Immunity to COVID-19 and De Novo Mutations}

The exhaustive and informative research report by Ng, Faulkner, Cornish, Rose, Harvey, Hussain et al. (2020) on pre-existing antibodies and de novo humoral immunity to SARS-CoV-2 in uninfected individuals covers a wide range of issues relating to the present wild-type and any future variations of the SARS-CoV-2 virus in humans. 
However, it does not seem to cover adverse reactions to de novo mutations, genetic alterations, or any variants of a virus that could be communicated for the first time through body fluids, including droplets and aerosols, to humans from infected humans or animals.

This could arise from the warp speed with which purportedly safe and effective vaccines are being clinically tested, which includes escaped vaccine mutants that could lead to more virulent strains, repeated infections, possible infections between the first and second jabs, and accumulated resistance to any vaccines over time.

\section{Frequency and Duration of COVID-19 Reinfection}

The interesting and challenging report by de Vrieze (2020) regarding possible reinfection after purported recovery from COVID-19 naturally leads to a number of unanswered queries:

(1) Is there any clinical trial evidence regarding the frequency and duration before reinfection from COVID-19?

(2) Losses of a sense of smell and hearing, as well as persistent fatigue, are some of the known long-hauler symptoms of COVID-19 infection.

(3) Is there any difference between the symptoms of long haulers and persistently infected patients, where the patient has not necessarily recovered from the disease?

(4) What immunity does infection from the disease provide, and for what duration?

(5) If immunity is indeed fragile, and wanes quickly, what is the probability that reinfection occurs, and what are the characteristics of recurrence?

(6) Is there any difference between the characteristics of long-hauler asymptomatic individuals and likely-to-be reinfected patients?

(7) What is the likely effect on the safety and efficiency of vaccines, especially with twojab vaccinations?

(8) Can any vaccines guarantee encouraging outcomes?

(9) In particular, can a patient be infected in between the two jabs?

(10) Are infections possibly misdiagnosed false positives, or were the previous results purportedly indicating recovery false negatives? 
(11) What are the symptoms arising from the original infection and reinfections, or possibly resurgence of the original infection?

(12) Are the differences in severity a function of the duration between the original infection and reinfection?

(13) Are reinfections similar to the wild-type coronavirus original infection, or possibly mutations that enhance the effects of COVID-19 and hamper recovery??

(14) Can infection from the SARS-CoV-2 virus increase the probability of being infected by other diseases?

(15) What effects can be expected regarding the frequency and duration of COVID-19 from economic, physical, and mental health care issues leading to stress, anxiety, self harm, and worse?

\section{SARS-CoV-2 Transmission Between Mink and Humans}

The detailed investigation by health service experts in Oude Munnink, Sikkema, Nieuwenhuijse, Molenaar, Munder et al. (2020) raises challenging issues regarding the transmission of the virus that causes COVID-19 between humans and mammals, specifically farmed mink.

There have been many mink infected with the virus in The Netherlands, Denmark, Poland, as

well as in Utah, Wisconsin, and Michigan in the USA, all of which have wiped out a high proportion of the breeding colonies.

Despite there being evidence of transmission from mink to humans, there is little available evidence of transmission from humans to mink.

Nevertheless, the possibility of such transmission is disconcerting, just as in the case of bidirectional transmission between humans and domesticated pets, specifically cats and dogs.

\section{Further Known Unknowns About T Cell Immunity to COVID-19}

The detailed and incisive explanation of known unknowns about $\mathrm{T}$ cell immunity in acute and convalescent COVID-19 infection by Karlsson, Humbert and Buggert (2020) is informative and helpful for medical specialists and every member of society. 
Further known unknowns include:

(1) Does $\mathrm{T}$ cell immunity to SARS-CoV-2 apply equally in acute and convalescent COVID-19 infection?

(2) How do preexisting conditions and comorbidities affect T cell immunity?

(3) What specific $\mathrm{T}$ cells might have a detrimental impact on the clinical outcome and contribute to long COVID (or long hauler) symptoms?

(4) What is the role of $\mathrm{T}$ cell immunity in separating long hauler COVID-19 from reinfection?

(5) How do T cells affect the likely duration between initial infection and reinfection?

(6) What are the different effects of $\mathrm{T}$ cell in vaccines in increasing protection against the virus for individuals who have not been infected, purportedly recovered patients, long hauler COVID-19, and reinfection?

(7) What are the different durations of $\mathrm{T}$ cell in vaccines in increasing protection against the virus for individuals who have not been infected, purportedly recovered patients, long hauler COVID-19, and reinfection?

(8) Is T cell immunity to COVID-19 affected by factors such as age, gender, socioeconomic inequalities, race, ethnicity, and general health conditions?

\section{Modeling Confusion to Clarity in a COVID-19 World}

The fascinating and informative editorial by two leading experts in science and technology, namely Press and Levin (2020), should be read by any modeller in epidemiology, biostatistics, statistics, econometrics, and psychometrics, among many others, especially those whose research work can help to save lives during and after a pandemic such as COVID-19.

Many models have predicted different waves of COVID-19 infections, some flat and others exponentiating, with a wide range of interpretations regarding flattening of the curve.

Access to timely high frequency daily medical and hospitalization data, as well as the inclusion of such data in recursively estimated and predicted models, would lead to finely attuned empirical analysis and associated interpretations and public policy decision making by the US CDC and NSF, among other highly-respected medical and scientific institutes. 
It is not always clear who might be asymptomatic carriers and transmitters of the virus, long haul purportedly recovered patients, and reinfection rates, according to medical, healthcare, and socio-economic conditions, among other known unknowns.

Epidemiological modeling and forecasting can be supported strongly by several other cognate technical disciplines, including mathematics, statistics, biostatistics, economics, econometrics, and computer science, that are concerned with specialized and robust models of event studies, policy interventions, and behavioural responses, using extremely large time series and cross-section panel data sets.

A coordinated strategy is essential for mitigating the numerous waves of the COVID-19 pandemic, which is likely to exist for many years to come, as well as preparing for future pandemics.

\section{Determining Serological Correlates of COVID-19 Severity}

The thorough and detailed study by Shrock, Fujimura, Kula, Timms, Lee, Leng et al. (2020), a group of medical specialists in an impressive collaborative research team, examines how investigating humoral responses to the SARS-CoV-2 virus that causes COVID-19 is essential for improving the specificity of diagnostic tests, therapy, and the search for a safe, effective, and durable vaccine.

A relatively small sample of 232 COVID-19 patients and 190 pre-COVID-19 era controls form the basis of the empirical analysis.

An important discovery is that patients with more severe COVID-19, predominantly males, exhibit stronger and broader viral responses, weaker antibody responses to prior infections, and a higher incidence of other viruses that were attributed to general demographic covariates.

Important correlations are found between COVID-19 severity, and both the history of exposure to the virus, and the overall strength of response to previous infections, subject to a variety of demographic and socio-economic conditioning factors, including age, gender, race, ethnicity, income, access to hospital and healthcare insurance, geographical location, among others.

The serological correlation of the degree of COVID-19 severity of infection with a host of observable factors is crucial in determining how to mitigate the spread of the virus, and the discovery of a safe, effective, and durable vaccine.

Correlation is dramatically different from causation, especially with different demographics of the hospitalized and nonhospitalized patients, as well as preexisting comorbidities. 
A larger sample with similar demographic considerations, as well as severe and mild forms of the disease, would enable a comprehensive analysis of the correlations, and possibly model the causation of mild and severe infections, against a range of measurable medical and socio-economic factors, to determine whether observed outcomes are a cause or consequence of the degree of COVID-19 severity.

\section{What Came First, Effective COVID-19 Vaccines or Trial Ethics?}

As discussed in Wendler, Ochoa, Millum, Grady and Taylor (2020), safe, effective, durable, affordable, and widely distributed vaccines are essential to mitigate, and possibly control, the COVID-19 pandemic.

If two injections are required for some vaccines, possibly over a period of 3 weeks, might a patient become infected between the first and second injections?

It is presumed that all essential ethical considerations have been satisfied before any clinical trials are conducted.

How is this possible when vaccines are being approved at warp speed, without any discussion

of durability, affordability, and wide distribution. Who is going to pay for the vaccines, federal, state or local governments, or patients?

\section{Safety et al. Requirements for COVID-19 Vaccines}

In addition to a safe vaccine for COVID-19 (see Haynes, Corey, Fernandes, Gilbert, Hotez, Rao et al. (2020)), it is also important that a viable vaccine is effective, tolerable, immunogenic, durable, affordable, ethically approved, widely distributed, and fast acting, preferably with a single injection to prevent infection between the first and second doses.

Otherwise, any intended global public healthcare approach to achieve herd immunity, mitigation, or unlikely elimination of the virus and associated disease will not be achieved.

\section{Controlling Transmission Heterogeneities and Controllability of SARS-CoV-2 and COVID-19}


As stated in Sun, Wang, Gao, Wang, Luo, Ren, Zhan, Chen et al. (2020), the connection between transmission heterogeneities is crucial examining the transmission from primary infections based on infectious disease dynamics.

The detailed, clear, and thorough empirical analysis and modelling provides interesting and informative reading for epidemiologists, biostatisticians, and healthcare experts, as well as nonspecialists.

The prescient comment on the duration of the lockdown period shows the possible effects of increased transmission risk within households, on the one hand, while isolation, quarantining, and lockdowns may reduce transmission risk, on the other.

The likelihood of lockdowns reducing transmission risk seems to ignore important aspects of mental health issues, as well as long hauler COVID-19 effects, including shortness of breath, perpetual tiredness, loss of taste, smell and hearing, and reduced life expectancy, among many other observed illnesses in purportedly recovered patients.

Accurate modelling and forecasting relies on access to accurate and detailed data, which may be available to the researchers of the important and informative report.

\section{Transmission Dynamics for a Seemingly Never Ending COVID-19 Pandemic}

In May 2020, as suggested in Kissler, Tedijanto, Goldstein, Grad and Lipsitch (2020), it might have seemed as though the COVID-219 pandemic was going to end sooner rather than later.

As the world approaches Christmas 2020 and New Year 2021, the seemingly never ending waves of the pandemic are difficult to predict.

To state the obvious, an increasingly large number of confirmed cases and deaths indicate that COVID-19 is now out of control, apart from some isolated countries territories.

The transmission curves are not flattening, there is no herd immunity, and the rejection of the best healthcare advice, including masking and social distancing, by so many in so many countries indicate it will be a long time before the pandemic is mitigated or controlled.

\section{Susceptibility to and Long Hauler Effects from Severe COVID-19}


Beck and Aksentijevich (2020) discuss the increasingly important issue of susceptibility to severe COVID-19.

Severe immunodeficiencies characterized by life-threatening viral infections, gene mutations, opportunistic infection, rare monogenic autoinflammatory and immunodeficiency disorders, impaired type 1 IFN responses, rare pathogenic variants, deleterious variants that can impair gene function, defects in type I IFN gene expression and protein levels demonstrating recurrent diminished activity of this pathway, inherited deleterious variants, and neutralizing autoantibodies (misguided antibodies), among other factors, seem to be hallmarks that define and determine susceptibility to severe cases of COVID-19.

Regardless of the numerous public announcements about the life-changing effects of any approved vaccines, the remaining long hauler effects such as advanced age, shortness of breath (dyspnea), chronic obstructive pulmonary disease (COPD), perpetual tiredness, chronic fatigue, which may be indicative of thyroid disorder, heart disease, diabetes, mitochondrial disease, sickle cell disease, chronic kidney disease, comorbidities, compromised immune systems, loss of taste, smell, hearing, and speech (ear, nose and throat otolaryngological side effects), reduced life expectancy, pregnancy, erectile dysfunction and reproductive health, and adverse effects on mental health, among others, will be permanent reminders of the dangerous virus and disease.

The effects and outcomes of COVID-19 can be severe in terms of both the medical conditions determining the risk of contracting the disease, as well as the long hauler outcomes when patients have purportedly recovered from COVID-19.

\section{Attack Rates of COVID-19 in the Brazilian Amazon}

The detailed and careful empirical investigation by an expert combination of epidemiologists, medical researchers, healthcare and infectious disease specialists, biostatisticians, and economists in Buss, Prete, Abrahim, Mendrone Jr, Salomon et al. (2020) is a welcome and informative contribution.

There is no doubt that Brazil is suffering greatly from the COVID-19 pandemic, behind only the USA and India in the number of confirmed cases and deaths.

The attack rate is an estimate of the final size of an unmitigated epidemic or pandemic, after correcting for cases without a detectable antibody response and antibody waning. 
It also provides a data-based estimate of the extent of COVID-19 spread in the absence of effective mitigation, such as a safe, effective, affordable, durable, widely distributed vaccine.

Manaus is the seventh largest city in Brazil, at over 2 million, whereas São Paulo is the largest

city in Brazil, and one of the largest conurbations in the world, with over 12 million in the city and over 22 million in the greater conurbation.

It is not surprising that São Paulo has a considerably lower attack rate than Manaus, capital city in the State of Amazonas, in the middle of the Amazon Rainforest at the confluence of the Rio Negro River and Solimões River, with both tributaries forming the Amazon River.

The availability of the higher quality of hospitalization and healthcare in the wealthy industrialized city of São Paulo, as compared with Manaus, is worth highlighting.

\section{Humoral Immunity to Severe COVID-19}

The expert pediatricians (see Fanous, Guerrero-Pena Pillai (2020)) argue that neutralizing antibody responses to SARS-CoV-2 have generally been assumed to be protective against COVID19, but with limited durability.

Humoral immunity is a function of B cells that bind to a specific antigen, against which it will initiate an antibody response.

There are many factors that lead to severe immunodeficiencies characterized by lifethreatening viral infections that seem to be hallmarks that define and determine susceptibility to severe cases of COVID-19.

However, neutralizing antibody responses do not yet seem to have been demonstrated to be protective against, or susceptible to, cases of severe COVID-19.

\section{Phylogenetic Analysis of COVID-19 Superspreading Events}

The impressive and exhaustive phylogenetic approach to the evolution of viral infectious disease, by a team of medical experts in Lemieux, Siddle, Shaw, Loreth, Schaffner et al (2020) of the evolutionary relationships among biological entities that underlie COVID-19, should be read by medical specialists and non-specialists alike. 
A small number of introductions of the virus was found to have led to most cases, with varying transmission dynamics in superspreading events that provide the link between transmission from a single source or individual clusters and a wider spread of secondary infections in the community.

With over 70 million cases of COVID-19 observed worldwide and over 16 million in the USA, and with deaths numbering 1.6 million and 300,000, respectively, as of 11 December 2020, superspreading events need to be controlled in order to mitigate the pandemic.

The critical discussion of sampling, statistical techniques, data collection, ancestral state reconstruction, and analysis of superspreading events is impressive.

It is worth noting that the numbers of cases linked to various superspreading events are based on estimates, so there are measurement errors in the generated data that might be accommodated in predicting the numbers of likely infections arising from future superspreading events.

\section{Seasonal Flu Versus Seasonal COVID-19}

A wide range of seasonal coronaviruses is examined in Guthmiller and Wilson (2020).

Seasonal flu patterns have been monitored for over sixty years across different continents since the first safe and effective monovalent flu vaccine was invented in 1938.

Is it possible that repeated exposure to seasonal flu will enable the production of cross-reactive antibodies and humoral immunity to mitigate infection from medium and severe SARS-CoV-2 through preexisting immunity according to age cohorts?

Is COVID-19 increasingly likely to remain in the population forever, much like the seasonal flu, but without any seasonal warnings, both before and after reportedly safe, effective, durable, affordable, and wide ranging vaccines are made available, with subsequent analysis of the antibody specificity induced by vaccination?

A minimum of one year is essential to be able to detect a seasonal pattern in any set of data, so it is still too early to infer any seasonal patterns or cross-reactive humoral immunity of the seasonal flu with COVID-19, and whether such interactions are likely to mitigate and protect, or exacerbate and accentuate, the effects of COVID-19, due to a lack of data availability and frequency.

COVID-19 cases have been monitored for less than one year, or four seasons, though seasonal patterns will be observed within the next year or two, with higher infection rates expected during the colder months. 


\section{Controlling the Spread of COVID-19}

The informative perspective by medical experts, Lee, Wada, Grabowski, Gurley and Lessler (2020), on the SARS-CoV-2 virus and COVID-19 disease evaluate how the virus and disease are spread.

Few countries and territories have effectively controlled the spread of the pandemic, including Australia, New Zealand, Taiwan, Hong Kong, South Korea, Finland, Norway, and Denmark, even though reintroductions are highly likely in areas with local elimination.

The perspective emphasizes that the fundamental engines driving the pandemic provide a foundation for interpreting and understanding any updates on the virus and disease.

Herd immunity is mentioned in passing, but no country has come close to the expected $80 \%$ or more of the susceptible population to develop an immune response,

As yet, little is known about the effectiveness or durability of the presumed immunity in order to focus on herd immunity as a public policy goal, though Sweden has shown that it can be far from effective and successful.

It is argued that close household contact and older adults are key drivers of transmission, with the known unknowns including seasonal variations, heterogeneities in the distribution of the population, symptomatic and asymptomatic ratios, superspreading, overdispersion in transmission, visitors to aged care facilities and prisons, duration of immunity, the rate of reinfections, and comprehensive long haul symptoms.

The known knowns to mitigate the spread of COVID-19 include social distancing, hand hygiene, and masking against droplets, and eye protection with goggles against infection through aerosols.

Otherwise, government intervention might be required to impose punitive extended lockdowns on the economy and society.

\section{Antibody Responses to Severe COVID-19}

The detailed and informative technical analysis by a group of medical experts, Röltgen, Powell, Wirz, Stevens, Hogan et al. (2020), on the duration of antibody responses to severe COVID-19, 
highlights specific antibodies that prevent viral spike receptors to neutralize the SARS-CoV-2 virus.

However, neutralizing the virus does not mean the serological responses that affect the clinical outcomes of infected patients are known.

SARS-CoV-2 antibodies targeting specific spike domains were observed in outpatients with mild illness, as compared with patients who had severe COVID-19.

Severe COVID-19 does not seem to be defined, though it is reported that 25 of 79 hospitalized COVID-19 patients died.

\section{Direct and Indirect COVID-19 Effects on the Heart}

The interesting, informative, and insightful perspective by Topol (2020) on how COVID-19 can affect the heart, both directly and indirectly, leads to questions as to whether similar effects occur for other primary internal and external organs other than the respiratory tract, such as the liver, kidney, brain, and ENT.

The SARS-CoV-2 virus has been identified as having "marked tropism for the heart and can lead to myocarditis (inflammation of the heart), necrosis of its cells, mimicking of a heart attack, arrhythmias, and acute or protracted heart failure (muscle dysfunction)", even for mild symptoms, or none at all.

It is crucial to determine how marked tropism, and other symptoms, for the heart might be affected by severe COVID-19, regardless of age, especially for patients with internal organ damage and other comorbidities.

As compared with SARS (or SARS-CoV) and MERS (or MERS-CoV), SARS-CoV-2 leads to more extensive cardiac involvement, and possibly also blood clots, which can lead to heart attacks and strokes, affect the lungs, heart, brain, and anywhere else the clot might travel through the blood.

COVID-19 would be expected to affect other internal and external organs, for which there are presently few observed clinical and non-clinical patient outcomes, besides the respiratory tract, and given the perspective, also the heart.

\section{Government Interventions Against COVID-19}


The impressive, comprehensive, and virtually exhaustive analysis by an impressive research team in Brauner, Mindermann, Sharma, Johnston, Salvatier et al. (2021) examined a major goal of governments, namely the effectiveness of interventions against COVID-19, which is intended to reduce the deleterious impact of the pandemic on the population.

Several European (34) and other non-European (7) governments that attempted to control the pandemic with nonpharmaceutical interventions (NPIs), between 22 January and 30 May 2020, were analyzed to determine the most effective NPIs.

Queries regarding the detailed empirical analysis include:

(1) An asymmetric Laplace prior ... [that] ... places $80 \%$ of its probability mass on positive effects was assumed arbitrarily.

(2) Although sensitivity analysis is undertaken, the absence of diagnostic checks of the underlying assumptions of the models makes it difficult to determine the robustness of the empirical analysis.

(3) As different countries implemented different sets of NPIs at different times and in different orders, a deeper analysis of pooling of the data would have been helpful.

(4) The generation interval was assumed arbitrarily to have a gamma distribution, with a mean prior of 5.06 days, derived from a meta-analysis, but it did not seem to be noted that the meta-analysis contains errors in data measurement.

(5) The arbitrary choice of a Bayesian hierarchical model that links NPI implementation dates to national case and death counts might usefully be tested against alternative models.

(6) The reported cases and deaths are assumed arbitrarily to follow a negative binomial output distribution with separate inferred dispersion parameters for cases and deaths.

(7) Each NPI, which was categorized as small, moderate, and large, was assumed arbitrarily to affect the reproduction number by a multiplicative factor, which would be worth testing against alternative assumptions.

(8) The sample data set might be extended beyond 30 May 2020 to determine the effects of the second and third waves of the pandemic on NPIs in November and December 2020. 
(9) The effects of droplets and aerosols might be investigated in a further analysis of an extended data set.

(10) The infection numbers through confirmed reported cases and fatal deaths are assumed arbitrarily to grow at the same daily rate, which might be tested with a further expanded data set.

(11) As NPIs are effectively event studies, a critical analysis of each event would be useful and informative.

(12) For each country, the data set started on 22 January and ended after the first lifting on an NPI, or on 30 May 2020, whichever came first, which seems to suggest that the data set was not identical for each country.

\section{COVID-19 Vaccines Should Be Celebrated With, By and For All}

The enticing and encouraging editorial by Thorp (2020) is significant in many respects, not only because of celebrating the breakthrough discoveries of vaccines that may be safe, efficient, durable, affordable, and widely available, but also in highlighting that the Science journal has made COVID-19 research free to read, without restriction, have expedited publication of research, and introduced the innovative eLetters to encourage researchers with no medical research qualifications to contribute to the discussion and analysis of COVID-19.

The safety, efficiency, and especially duration of many vaccines have yet to be determined, but any new discoveries will hit the leading journals at the very earliest possibility.

In addition to many disciplines not necessarily having contributed directly to the discovery of any of the multitude of vaccines, many researchers in non-medical scientific research have analyzed the direct and indirect global socio-economic, political, and demographic effects of COVID-19, and how the costs of invention in R\&D have affected society, have come from the sciences, social sciences, business, and humanities.

In a combined multidisciplinary attack on the COVID-19 pandemic, the global scientific

community has contributed to mitigating the virus and disease, and brought a shining light to a comparatively bleak 2020 leading into an undoubtedly more enlightening New Year.

\section{Countering the Division on COVID-19 and Mutated Strains}


The heartening viewpoint by Kupferschmidt (2020) promotes science as an anti-divisive argument against the COVID-19 pandemic that is threatening to create chaos of the mind globally.

As if the COVID-19 disease arising from the SARS-CoV-2 virus were not bad enough, news regarding a mutation strain of SARS-CoV-2 has just been discovered in London, which is even more highly transmissible and infectious than the original wild-type COVID-19 strain.

This is especially so if the World Health Organization was aware of the mutation months ago.

If the mutation is more highly transmissible, there will be many more infections of COVID19 , and hence also many more deaths attributed to it.

Moreover, any authorized vaccine for the wild-type coronavirus that is presently being distributed in some countries might not be as safe and effective for highly transmissible mutations, or indeed for any mutated strains.

It is one position to disagree on interpretations of scientific evidence, but another altogether to ignore completely the scientific evidence that is available, for whatever reason, politics being one of the more common causes of dissension.

If society does not respect and accept scientific advice, it will rely on the abundant provably false "fake news" and misinformation that dominate social media, both about the pandemic and the numerous vaccines that are purported to mitigate its uncontrolled spread globally.

\section{Encompassing Tests for COVID-19}

The pointed and informative perspective by medical experts in Mina and Andersen (2020) against a one-size fits all testing strategy for COVID-19 bears testimony that a flexible approach to the pandemic is necessary, specially with the recent developments regarding a mutation strain of SARS-CoV-2 in the UK, which is even more highly transmissible and infectious than the original wild-type COVID-19 strain.

It stands to reason that any authorized vaccine for the wild-type coronavirus might not be as safe and effective for highly transmissible mutations, which arise as the virus replicates, and which affects risk monitoring and management, diagnostic testing, medical data collection and analysis, and global vaccination programs.

The mutated variant has been given different names, such as a VUI (Variant Under Investigation) 202012/01, VUI-202012/01, or the B.1.1.7 lineage. 
Accurate testing for the wild strain will not necessarily lead to the same outcomes as for the mutated strains, especially in terms of determining false positives and false negatives.

Mathematical and statistical modelling of herding effects can lead to inaccuracies, especially with wide bounds on any parameter estimates of the model, including the precise \% of a population that needs to be infected for the model to work in practice as it is hypothesized to work in theory.

Whatever its identification, mutations should not be treated as if they were treatable in the same manner as the original wild strain for which vaccines have been created and are being distributed in several countries.

\section{Positive Messages Give Hope, Even in a COVID-19 World}

The positive, encouraging, and reassuring news in Bouveret (2020) gives hope for the future to all who listen.

Without hope, all is lost, especially in a COVID-19 world.

The year 2020 will be imprinted on the minds of many for the COVID-19 pandemic, but it also gave time to reflect to all who were isolated physically, quarantined, or affected by severe lock downs.

No one wants to be given a cancer diagnosis, especially if the prognosis is stage 4, aggressive, and inoperable, even if treatable, but positive benefits come from the way one might respond to it.

Pain can be difficult to deal with, but expert pain management and searching for the wonderful aspects in our daily lives can mitigate what can become a long term an annoying side effect of any serious life-threatening illness.

Enjoying the success of others can prove fulfilling for everyone, even turning 40, which is not old for a tree, even in the time of COVID-19.

\section{How Safe, Effective, Durable, and Affordable Are Warp Speed COVID-19 Vaccines?}

As stated in Cohen (2020a), there is no question that vaccines for COVID-19 developed at warp speed are a breakthrough for science and everyone who will be vaccinated against the pandemic, but how safe, effective, durable, affordable, and widely distributed are they? 
The sensitive, encouraging, and reassuring news item provides a positive spin on the genuinely innovative breakthroughs, but how do the approved vaccines perform against not only the wild type original virus, but also against various mutant strains that are being uncovered?

However, what are the treatments that are available for many individuals in society across all age groups who are suffering from mental stress, anxiety, loneliness, depression, self harm, and worse?

Moreover, how accurate are the purported success rates of the warp speed approved vaccines that are being heralded by the companies producing the vaccines through news and social media, as possible saviours that will mitigate the dangerous effects of the virus and disease?

Without careful and detailed explanations as to who was selected to participate in the numerous laboratory clinical trials, their impact on members across all segments of society will need to await detailed empirical analysis after a wide distribution of the available vaccines, including long haul (or lifelong) COVID-19 patients and those with a wide range of existing comorbidities.

Remarkably innovative medical science discoveries have been made at warp speed as the pandemic continues to ravage the globe, but it is hoped that the possibly deleterious side effects of the vaccines to the real world, and their safety, effectiveness, and durability against a range of mutations (such as VUI-202012/01, or B.1.1.7, B.1.177, N501Y, 501Y.V2, 69-70del, and their various mutations), that are being encountered, albeit not at warp speed.

\section{Correlations of Severe COVID-19 Viral Responses}

The impressive research team of medical experts in Larsen, de Graaf, Sonneveld, Plomp, Nouta, Hoepel et al. (2021) has provided a comprehensive investigation of the effects of high levels of afucosylated IgG antibodies against SARS-CoV-2, amplifying pro-inflammatory cytokine release and acute phase responses, in critically ill COVID-19 patients, but not those with mild symptoms, which showed that antibody glycosylation plays a critical role in immune responses to enveloped viruses, including COVID-19.

This is an important empirical discovery for critically ill COVID-19 patients, primarily those with severe airway inflammation, leading to acute respiratory distress syndrome (ARDS), often leading to ICU admission or a fatal outcome, or severe enveloped viruses, such as SARS-CoV-2, with associated life-threatening severe COVID-19 infection. 
As is standard, four different groups of increasing disease severity were considered, namely outpatient, admit, ICU, and death, giving a series three well-defined categories of increasing severity compared with non-hospitalized outpatients.

The negative correlation between IgG-Fc galactosylation and advanced age, and the positive or negative correlation between afucosylated IgG antibodies and advanced age would be useful in the empirical analysis as conditioning factors based on genetics or stochastic triggers that might presently be unknown.

It would also be informative to determine whether the correlations were static or dynamic, whether IgG-Fc afucosylation results in potent immune responses, and if afucosylation of anti-S IgG might contribute to the exacerbation of COVID-19 in some patients, leading to ARDS, all of which are associated with enhanced protection, especially through approved vaccines.

The effects of these conditions would be significant in analyzing whether the approved vaccines are likely to work against the increasingly numerous known mutated strains of the original wild-type coronavirus.

\section{Known and Unknown Long Haul Effects From COVID-19}

The detailed and informative long haul effects from COVID-19 reported and featured in Couzin-Frankel (2020) in early August 2020 can be supplemented by:

(1) persistent loss of taste;

(2) persistent loss of hearing;

(3) persistent damage to memory;

(4) damage to the lungs;

(5) greater likelihood of Parkinson's disease;

(6) increased likelihood of mental healthcare issues to patients;

(7) increased likelihood of mental issues to frontline healthcare workers and carers;

(8) unknown impact on chronic diseases;

(9) unknown interactive effects with pre-existing comorbidities; unknown duration of any of the above. 


\section{How Should Approved COVID-19 Vaccines Be Distributed Globally?}

The World Health Organization (WHO), among others, has stated that approved vaccines that are safe and effective, and presumably also durable, affordable, and available, should be distributed equitably worldwide.

It is not entirely clear how approved vaccines will be distributed globally, although the accounting term, "First In, First Out (FIFO)", seems to be a common strategy, whereby commitments need to be made early in order to gain access to the vaccines.

Given this strategy, even relatively wealthy nations have had to wait in line to gain access to approved vaccines, as discussed in Cohen and Kupferschmidt (2020).

For nations that have gained access to such vaccines, the most vulnerable members of society, together with frontline healthcare and other essential workers, have been at the head of the queue in being vaccinated.

Vaccines that require a booster shot 3-4 weeks after the initial injection will probably require twice as many capsules to be purchased.

Herd immunity is the ultimate goal of vaccinations against COVID-19 infections, but the proportion of the population that needs to be vaccinated and/or infected is presently unknown.

\section{Anaphylactic Reactions to Pfizer's COVID-19 Vaccine}

Any vaccine, especially during the COVID-19 pandemic, should be safe, effective, durable, affordable, and available as minimal regulatory requirements.

According to the informative and penetrating investigation by de Vrieze (2021) that reports:

"At least 12 people suffered an anaphylactic reaction after receiving Pfizer's COVID-19 vaccine",

how could the vaccine have been approved as safe, especially given the widely self-reported success rates by Pfizer and BioNTech of around 95\% in the news and social media?

The cause may be due to the compound polyethylene glycol (PEG) in their vaccine, which is also contained in the vaccine produced by Moderna. 
Anaphylactic reactions are frequently caused by bee stings, as well as eating peanuts and tree nuts, so the compound must have been known to cause similar reactions in vaccinated individuals, regardless of whether it has previously been used in vaccines.

As it was purportedly the first time PEG was used in a vaccine, greater scrutiny should have been instigated and investigated in clinical laboratory trials before the vaccines were submitted for regulatory approval, especially as individuals with known previous allergic reactions were excluded from any clinical trials.

Any unapproved vaccines that are being peddled globally should be a cause of serious concern to the individuals, many in poor countries, who are given the vaccine to act as guinea pigs for the unapproved vaccines, especially if they are in the vulnerable category, such as the aged and very young.

\section{Open Access Is Excellent, But Who Should Pay, Especially During COVID-19?}

Open access publishing is discussed in detail in Brainard (2021).

Open access is an excellent idea, except for the arbitrary issue as to who might be paying for such access, especially during COVID-19.

Even leading journals such as Nature and Science have open access options, but the fees are extremely high.

Academics have been very slow in demanding that publishers should compensate them for the published articles.

Although Editors-in-Chief might be compensated for their contributions to managing journals, the forgotten reviewers should also be paid for their time and effort in enabling papers to be published, though this does not seem to have been mentioned in the excellent report.

\section{Fast Spreading COVID-19 Mutations}

The discussion in Kupferschmidt (2021a), regardless of whether the mutant strains of COVID19 arose from the UK, Spain, South Africa, or anywhere else, is alarming because the approved vaccines were clinically tested before the mutations were discovered. 
As if the increased transmissions, infections, and associated deaths arising from COVID-19 were not bad enough, initial news suggested that the UK mutation, SARS-CoV-2 VUI 202012/01 (or B.1.1.7) was far more infectious than the original wild-type form.

Viral evolution can be modelled if there is a consistent pattern to the changes, but COVID-19 seems to be a difficult disease to analyze, especially in terms of the as yet unknown number of mutations that can be ascribed to it, especially as the number and speed of mutations might increase with the number of infections.

Whether the mutations might lead to difficulties in increasing the number of infected individuals as well as the number of vaccinated people in the population to achieve herd immunity, only time will tell.

\section{The Reality of COVID-19 in Africa}

The informative perspective by Maeda and Nkengasong (2021) tackles the issue of the purportedly puzzling COVID-19 pandemic in Africa to determine what is real and what is not.

It would be simplistic to suggest that the data on confirmed infections and deaths are inaccurate through poor methods of data collection and inadequate testing for the virus and disease, as it may well be in several continents, but it can also be explained in part by decisive measures taken early to prepare Africa for the unknown future that was about to destabilize the world.

As in many countries, there is a need to balance opening the economy and society against mitigation of the disease.

The perspective makes a number of puzzling statements without proof, namely:

(1) limited testing limits the trajectory of COVID-19, with omission of "purported" before trajectory as it is, in fact, biased downward by the lack of testing;

(2) climatic differences, when there are many regions globally with similar climates to various regions in Africa;

(3) preexisting immunity, which ignores where SARS and MERS were discovered in regions outside Africa;

(4) genetic factors, which is a misunderstood and misleading interpretation of nature versus nurture (aka genes versus environment). 
Only time will tell how accurate or misleading the data from Africa on COVID-19 might be.

\section{How Does COVID-19 Affect Cancer and its Treatment in Africa?}

\section{"It was the best of times, it was the worst of times" Charles Dickens, A Tale of Two Cities, 1859}

In the incisive perspective by Addai and Ngwa (2021), the connection between COVID19 and various types of cancer are discussed by medical experts, with an emphasis on discussing the treatment of breast cancer in Africa.

How medical oncologists treat various types and stages of different cancers is fraught with difficulty at the best of times, but is made more difficult with medical healthcare availability and associated hospital and medical insurance costs when hospital beds and frontline healthcare workers are physically and mentally overworked because of the need for beds and ICU facilities for COVID-19 patients.

How does the relationship between cancer and its treatment, including prevention and palliative care, differ with the presence of the COVID-19 pandemic in Africa, especially with the reallocation of medical facilities, treatment, and advice, including cancelling cancer operations altogether, especially for those with low priority?

Shutting national borders, grounding airlines, and limiting national and international travel at the onset of the COVID-19 pandemic affected oncology and cancer treatment, early detection, and screening, dramatically in the continent, with the diagnoses of more advanced cancers as unavoidable outcomes.

With the lack of universal health coverage, and virtually no access to private healthcare insurance coverage, socio-economic and demographic considerations become increasingly important for genetic diseases such as cancer, especially with the prevalence of COVID-19.

As patients with concurrent chemoradiotherapy are susceptible to increased risks of contracting COVID-19, this is another reason for reducing access to chemo and radio therapies in the presence of a more life-threatening COVID-19 disease. 
Reduced palliative care can lead to greater mental health issues, such as loneliness, stress, and anxiety, which can worsen an already distressing situation for patients and their carers.

\section{Duration of Immune Memory to COVID-19}

As discussed in Dan, Mateus, Kato, Hastie, Yu, Faliti et al. (2020), the immune memory of the SARS-CoV-2 virus is essential for determining the effectiveness and duration of any approved vaccines for the COVID-19 disease.

The detailed, comprehensive, and informative research findings by a team of experts regarding immune memory to the virus in 254 samples from 188 recovered COVID-19 cases in the USA, including 43 samples at up to 8 months post-infection, gives a more accurate indication of the duration of immune memory than for shorter time periods.

The research findings are based on an evaluation of the various components of the virus, including different cell types, as well as various degrees of severity of the disease, in order to investigate the durability of protection against viral reinfection of COVID-19.

Bearing in mind that there are no defined mechanisms in humans of long-term protective immunity, important findings suggest that $\mathrm{T}$ cell memory might reach a slower decay phase after the first 8 months of post-infection, though a proportion of the infected population with low immune memory might suffer reinfection.

Durable immunity in order to approach herd immunity would require a high proportion of the population to be infected and/or inoculated against the disease, which will depend on the duration of immune memory.

An issue that deserves attention in further studies is the effect of mutated strains of the virus, such as those from the UK and South Africa, and possibly Spain and elsewhere, on the duration of immune memory and the effectiveness of any approved vaccines.

\section{COVID-19 Mutations and Vaccinations}

As discussed in Kupferschmidt (2021b), if the original wild-type COVID-19 disease were not bad enough, along come several mutations from the UK and South Africa, and possibly elsewhere, that make global transmission, infection, and deaths inevitably increase, and render the safety, 
efficacy, duration, affordability, and availability of approved vaccines, and attaining herd immunity, that much more difficult.

Do these mutations herald a new wave and extension of the pandemic, and will the purported properties of the currently approved vaccines be affected by the mutated strains?

Does the dramatic increase in all the negative aspects of COVID-19 in the USA suggest the existence of a new US variant of the virus and disease, as speculated by Dr. Deborah Birx of the White House coronavirus task force, a statement that was criticized by the US CDC as "inaccurate"?

Does the cold weather in the northern hemisphere's winter decrease immune systems which, together with other ailments, facilitate the spread of the disease, and alter the viral intensity?

Moreover, might the $\%$ of the population to be infected by COVID-19, or the $\%$ of the population that requires vaccination, in order to achieve herd immunity, affect the presently unknown threshold?

It would bode well to heed the prescient advice that no one can be ahead of the virus and disease as COVID-19 is in charge.

\section{COVID-19 Lineage Dynamics, Mutations, and Vaccines}

As discussed in du Plessis, McCrone, Zarebski, Hill, Ruis et al. (2021), the number, size, duration, and spatial distribution of dynamic lineages can be used to examine the rapid fluctuations in viral importation and transmission rates during lockdowns in order to analyze the COVID-19 transmission dynamics and to eliminate the lineages.

The detailed and comprehensive analysis by an impressive team of medical experts examines how co-circulating transmission lineages vary in size, distribution, and persistence, and how to evaluate their combined effects on epidemic size and duration.

Such research is essential as the structure of small-scale transmission clusters cannot be extended in any practical sense to highly dynamic pandemics, and is based on all of the large number of SARS-CoV-2 genomes available before 26 June 2020.

It would be informative to investigate how the original wild-type viral strains might have affected the genetic structure and lineage dynamics, especially in light of planning and evaluating public healthcare strategies regarding the following: 
(1) extending the data set beyond June 2020;

(2) the presence of second and higher waves of infections in the UK and globally, especially from November 2020;

(3) the various approved COVID-19 vaccines that are presently being distributed globally;

(4) the discovery of more highly transmissible and infectious mutated viral strains of the virus and disease;

(5) analyze the different regions geographically in a more detailed manner to expand the structure of small-scale local transmission clusters;

(6) evaluating the interconnections of local transmission clusters geographically;

(7) modelling the size dependence and other parameters using time series data;

(8) evaluating the aggregated effects of multiple heterogeneous lineage-specific patterns;

(9) analyzing the dynamic connection between domestic and international transmission lineages;

(10) determining whether lockdowns work in a more general sense than in the analysis of viral importation and transmission rates;

(11) with numerous international arrivals affecting the establishment and co-circulation of a large number of transmission lineages, might reductions in travel numbers be used to curtail the dynamic lineages;

(12) examining whether the geographic travel sources, histories, and seasons have any effect on the lineage dynamics.

\section{COVID-19, Immunity, and Endemicity}

In a fascinating research report by Lavine, Bjornstad and Antia (2021), the expert authors evaluate data on endemic human coronaviruses, and show that infection-blocking immunity wanes rapidly, but disease-reducing immunity does not.

It begs the question as to what is the best strategy for transitioning from a pandemic to a locallycontained endemic, possibly based on a high threshold of the population having been reached for viral infections, or a successful vaccination program, or both, to achieve herd immunity?

This leads to the following issues: 
(1) How does viral evolution develop?

(2) Do mutated strains become more resistant to currently approved vaccines?

(3) Can vaccines be adapted to deal with the more highly transmissible and infectious strains or variants that have been discovered in the UK and South Africa?

(4) Will the mutated strains lead to variations in the percentage of the population that is asymptomatic?

(5) Furthermore, will the mutated strains lead to variations in the percentage of the population that might suffer from long haul COVID-19 effects?

(6) How large are the regions for purposes of defining endemicity, such as city, state, province, prefecture, country, sub-continent, continent, or hemisphere?

(7) How does the SARS-CoV-2 virus that causes COVID-19 differ from SARS (or SARSCoV-1) and MERS?

(8) Can specific immunity types be predicted in the short run and long run?

(9) Can the duration of a pandemic such as COVID-19 be predicted?

(10) Can the duration of the effectiveness of alternative approved vaccines be predicted?

(11) At what point will the age distribution of infected cases differ from that of the population?

(12) What happens if older individuals have not acquired immunity through having been infected, vaccinated, or both, at an early age?

(13) How many times will an individual need to be infected to acquire immunity from the virus?

(14) How many times will an individual need to be vaccinated to acquire immunity from the virus?

(15) What are the interactive effects of infection and subsequent vaccination, or the reverse?

(16) What is the likely duration of the transition from a pandemic to an endemic status, and how long will endemicity last?

(17) What are the effects of existing comorbidities on the transition to, and duration of, endemicity?

(18) What are the effects of the age structure on the transition to, and duration of, endemicity? 

pandemic and endemic states?

(20) Are the properties of the mutated strains likely to be different in the pandemic and endemic states?

\section{T Cells, Immune Systems, and Aging Hosts in a COVID-19 World}

As reported in Billi (2021), regulatory T cells are supposed to help protect the body against infection by regulating immune responses to infected and immune cells.

However, aging can lead to a weakening of the immune system, so that $\mathrm{T}$ cells can lead to inflammation rather than regulating it.

Aging tends to reduce protection against infection, may increase incidences of diseases, and lead to "traitorous" $\mathrm{T}$ cells, as discussed in the informative research report.

If the results in the report were extended to infections in a COVID-19 world, analysis of the following would be immensely important for diagnostic research and healthcare public policy:

(1) infection known knowns: short term effects on the lungs, heart, brain, and liver;

(2) symptomatic known knowns: ageusia and anosmia, persistent coughing, and high temperature;

(3) mutation known unknowns: existence of original wild-type coronavirus, and discovery of more highly transmissible and infectious mutated strains and variants;

(4) vaccine known unknowns: differential efficacy of approved vaccines for the original virus and its mutated strains and variants.

\section{Social Robots Assisting Humans in the COVID-19 Era}

The novel, informative, entertaining, and interactive focus by Scassellati and Vázquez (2020) highlights how innovative interdisciplinary social robotics can assist humans in a world that is presently being controlled by the COVID-19 pandemic.

Although reported exactly 6 months ago at barely the mid-point of the ongoing pandemic, before the second and higher waves of infections, the discovery and distribution of approved 
vaccines, and the developing more highly transmissible and infectious mutated strains of the original wild-type coronavirus, the prescient emphasis on social distancing, mental healthcare, distance education at all levels through video-teaching and teleconferencing, and economic recovery, are all the more important in the current environment as the pandemic continues exponentiating in terms of infections and deaths.

Masking, hand hygiene, COVID-19 fatigue, short term and long haul symptoms, including monitoring and managing mental healthcare issues, and the safety, efficacy, and duration of vaccines, could all be added to the impressive list of issues that can be assisted by robots through repeated scientific and social media communication and surveillance through commentaries and videos.

Carefully planned and engineered robots can be directed toward commercial outlets, including ordering food, drinks, non-perishable items and many other products online, and to dynamic human environments to extract the most beneficial social outcomes for all.

\section{Herd Immunity Against COVID-19 Through Infection or Vaccination}

In Sridhar, D. and D. Gurdasani (2021), the expert authors caution against trying to achieve herd immunity through infection by COVID-19.

Herd immunity through infection has been an unmitigated failure in Sweden in terms of high infection and mortality rates, especially for the aged, because it has not infected a sufficiently high $\%$ of the population, aka "threshold", although it has tried this strategy since the start of the pandemic.

A recent study in Manaus, the capital of Amazonas state situated at the centre of the Brazilian Amazon, shows that herd immunity was not achieved even with an estimated threshold of $76 \%$.

It should be noted that the threshold is an unknown parameter, and has been discussed widely as being at least $70 \%$ or $80 \%$, which is in the ballpark of the estimated threshold in Manaus.

However, the duration of the estimated threshold can also be important, with the example of Sweden being a concentrated, and ultimately unsuccessful, national attempt, with approximately $50 \%$ of the infections being in the capital city of Stockholm.

On the other hand, Manaus is an isolated city at the centre of the Brazilian Amazon, nowhere near other population centres like São Paulo, Rio de Janeiro, and the capital city of Brasilia, with 
the estimated threshold increasing from 66\% in June 2020 to $76 \%$ in October 2020, which are not necessarily statistically significant differences.

Since the interesting research was conducted, approved vaccines have been distributed globally, and mutated strains and variants from the UK and South Africa, possibly among others, have been detected.

No clinical trials have yet been investigated about the safety, efficacy, and duration of the vaccines against the mutated strains and variants, which is problematic as herd immunity may also be achieved through vaccination programs, although the threshold $\%$ of the population that needs to be vaccinated is a known unknown.

\section{Cross-reactive Immunity to Zoonotic Diseases Like COVID-19}

As discussed in the impressive and informative research report by a team of medical experts in Cohen, Gnanapragasam, Lee, Hoffman, Ou, Kakutani et al. (2021), calamitous coronaviruses, including SARS-CoV-2 that causes COVID-19, are among the most serious zoonotic viruses that are shared between animals and humans.

Cross-reactive immunity responses were investigated for mice which shared, in some experiments, similarities with some previous results on such immunity for humans, although general conclusions were not possible to determine.

Zoonotic viruses may also be providing immunogenicity against a variety of human coronaviruses, including COVID-19, or emerging coronaviruses that have the potential to spillover to humans.

It is not presently known if immunization with certain types of nanoparticles will provide protection against a variety of known and emerging coronaviruses, including mutated strains and variants of the original wild-type coronavirus, such as those from the UK, South Africa, and possibly also from Spain, Brazil, the USA, and elsewhere.

Given the multiple mutations of coronaviruses, potential pan-coronavirus (pan-CoV) vaccines will almost certainly be needed to provide protective immunity against a combination or all of the multiple mutated strains and variants.

There seems to have been virtually no clinical trials of the currently approved vaccines against the known mutations of COVID-19, so pan-Cov vaccines against newly discovered zoonotic 
coronaviruses might take a considerable amount of time before they are developed, and proved to be safe, effective, durable, affordable, and available for distribution.

\section{Multivalent Nanobodies, Neutralization, and Suppression of COVID-19 Escape Mutants}

The detailed, comprehensive, and informative research report by a large team of medical professionals in Koenig, Das, Liu, Kümmerer, Gohr, Jenster et al. (2021) investigated how structural multivalent nanobodies can block infection from the SARS-CoV-2 virus that causes COVID-19, and also suppress the creation of mutated strains and variants (aka "escape mutants").

This is especially topical in light of the recent discoveries of highly transmissible and infectious mutations in the UK, South Africa, and elsewhere.

The experts engineered multivalent nanobodies, and showed that they had considerably greater neutralization as compared with monovalent nanobodies.

Nanobodies are also suitable for passive immunization against COVID-19, and are amenable to engineering, which should prove fruitful in suppressing escape mutants.

The preliminary experimental findings should prove encouraging to medical specialists, researchers, healthcare professionals, and public policy advocates about COVID-19, and how mutant strains and variants might be tackled to mitigate the ongoing pandemic.

Safe, effective, durable, affordable, and available vaccines, and alternative treatments, for immunocompromised and high-risk patients, children, infected patients who have purportedly recovered from the disease, and long haul COVID-19 sufferers, also need to be fast tracked.

\section{Public Debate About Science is Essential in a COVID-19 World}

The reassuring and encouraging editorial by Thorp (2021a) makes it clear that openness, accountability, transparency, and scientific evidence are not only good and essential for science and researchers to progress in all of the disciplines, but also to everyone, regardless of their academic discipline or educational background. 
"Alternative facts" and "fake news" in social media have held sway for several years, especially during the Trump administration, and science has been openly, dismissively, and unconscionably derided with little recourse by way of defense.

Leading research, public policy, and research institutes, including the US CDC, FDA, and a host of others, have been politicized, with little respect for scientific evidence and accuracy.

The Science journals have been consistently open and encouraging in publishing numerous research and healthcare policy papers that have defended such institutions, especially during the onset of the COVID-19 pandemic.

As discussed in the prescient editorial, masking and the choice and use of approved vaccines have led to different interpretations and recommendations among scientists.

The escaped mutants of the original wild-type coronavirus and disease, and predictions about how currently approved vaccines might be affected in terms of their safety, effectiveness, duration, affordability, and distribution, require the outcomes of clinical laboratory trials that have not yet been established.

Scientific discourse cannot be orchestrated in an open society, where public acceptance of scientific debate can be confusing, even to well-trained experts, and even lead to further debate and confusion.

The creation of the online eLetters forum that fosters ongoing peer review, including comments on editorials by individuals with little or no medical or other credentials, is a clear indication that scientific discussions must always remain balanced and logical, with benefits for the international community.

\section{Scientific Reporting on COVID-19 in China}

Normile (20121) discusses scientific reporting on the COVID-19 pandemic.

Where, when, and how the SARS-CoV-2 virus and the COVID-19 disease it caused might have started, the first reports came from the city of Wuhan toward the end of 2019.

Regardless of the information that was available in China, it was far more significant than what was available even to the World Health Organization (WHO) and any other institutions globally. 
Therefore, it should not be surprising that two institutes in Wuhan published a large number of papers in the first six months of the pandemic, though the output has not necessarily been sustained thereafter.

Significant advances in science depend on openness, information sharing, intellectual freedom, unmuzzled media, accountability, peer review, and evaluation by international experts, including by the WHO, none of which seems to have occurred in China to date, including COVID-19.

Publications in The Lancet in January 2020, regardless of the number of citations they might have attracted, would border on meaningless and uninformative as little was known by any medical specialist about the virus, whether inside or outside China.

Excluding Taiwan from the WHO, especially when it is clear that Taiwan has handled the pandemic as well as any country or territory, with the possible exception of Australia, New Zealand, Vietnam, and some Pacific island countries, would not seem to be in the best interests of the global community.

Scientific turning points are based on sharing scientific information, which works both ways.

New waves of infection, as well as escaped mutant strains and variants, are being discovered unexpectedly and suddenly, and much research still needs to be done.

Vaccines need to be approved as safe, effective, durable, affordable, and available, but China, as well as other countries, including Russia, have not yet made such data available.

This takes us back to the underlying requirements for significant advances in science to flourish.

\section{Circadian Rhythms and Social Jet Lag in COVID-19}

The informative report by Hurley (2021), an expert on circadian rhythms and social jet lag, explains much to non-experts as to why they are experiencing the following in pre-and duringCOVID-19:

(1) discrepancies between internal and external sleep-wake rhythms;

(2) variations in the number of hours of sleep;

(3) mental health issues;

(4) different waking hours;

(5) use of alarm clocks. 
Additional issues pre-and during-COVID-19 include:

(6) dream patterns;

(7) work behaviour;

(8) existing comorbidities;

(9) effects of approved vaccines;

(10) the prevalence of escaped mutants.

\section{COVID-19 Social Control Measures Should Have Been Implemented Years Ago}

As discussed in Servick (2021), COVID-19 control measures have suppressed seasonal flu infection for good reason, and should have been implemented years ago.

As discussed in the informative report, the social control measures that have emphasized personal hygiene, masking, social distancing, self isolation, quarantining, lockdowns, and much more, have mitigated the spread of COVID-19 and, in the process, dampened the effects of the seasonal flu.

However, the seasonal flu typically does not lead to a pandemic that affects the global economy, and generates calamitous physical and mental health illnesses, including long haul effects after purported recovery, as does COVID-19.

There are approved vaccines for the seasonal flu that are safe, effective, durable, affordable, and available, though their production during the pandemic has not been advertised widely.

The number of highly transmissible and infectious escaped mutants does not affect the seasonal flu to the degree it has for the effectiveness of approved vaccines for the emerging mutated strains and variants of the SARS-CoV-2 virus that causes COVID-19.

A known unknown is whether infection and purported recovery from mild, medium, or severe COVID-19 will provide any protective immunity against the seasonal flu, or if their intersection might make a combined infection even more serious, if that were at all possible.

\section{Tackling Student Mental Healthcare, Especially During COVID-19}


The caring, sensitive, and encouraging editorial by Leshner (2021) on student mental health and well-being by a leading expert should be embraced by all members of society, especially those who have the responsibility to nurture and protect students in dispensing knowledge, education, and physical and mental healthcare.

This holds all the more so during the COVID-19 pandemic, where social distancing measures, self isolation, quarantining, and lockdowns, especially for extended periods, have exacerbated and accelerated existing anxiety, mental stress, depression, self harm, substance abuse, and much worse.

The standard operating procedures on many campuses need to undergo a dramatic change and improvement to balance competitive frameworks that incorporate more balanced strategies and enable access to mental healthcare professionals and treatment.

Highlighting the importance and prevalence of mental health illnesses is the duty of the institution and teachers, with societal acceptance and encouragement as essential components of addressing the global health problem.

\section{Modelling Prioritized COVID-19 Vaccine Strategies}

It takes a multi-talented team of experts, as in Bubar, Reinholt, Kissler, Lipsitch, Cobey, Grad and Larremore (2021), to model the various phases of the COVID-19 pandemic, including a mathematical model that is based on five age-stratified vaccine prioritization strategies.

Achieving herd immunity requires either a high proportion of the population to purportedly recover from disease infection, or a high proportion of the population to be protected through treatment by approved vaccines that are safe, effective, durable, affordable, and available.

When production is limited, at least temporarily, the prioritization of vaccines is essential, and the optional strategies may be based on a number of factors, including cumulative incidence, mortality, and years of life lost.

Protection against infection can be undertaken directly through vaccination of the most vulnerable at risk individuals in society, or indirectly by vaccinating those who are most likely to transmit and infect others, an optimal balance between which is a primary aim of mathematical modelling. 
Socio-economic inequalities and the absence of fairness to many disadvantaged members of society are difficult to model as many alternative scenarios are available.

Social protection measures, such as social distancing, masking,self isolation, quarantining, and lockdowns can also be modelled in a number of alternative scenarios.

Nevertheless, due diligence and the use of diagnostic checks to evaluate the robustness of any empirical outcomes are always recommended.

The recent discovery of escaped mutants, such as those from the UK and South Africa, and possibly also from Brazil and the USA, that are more transmissible and infectious than the original wild-type SARS-CoV-2 virus that causes COVID-19, would also affect the optimal balance between the direct and indirect methods of protection through the approved vaccines.

Vindication through observed empirical data is the true test of the accuracy of any mathematical or statistical model, as well as predicting the likely future trajectories of trying to achieve herd immunity.

\section{Optimal Vaccination Strategies for COVID-19}

The informative and prescriptive perspective on optimal vaccination strategies for COVID-19 by Fitzpatrick and Galvani (2021) has the ringing inference that one size does not fit all.

Several safe, effective, durable, and affordable vaccines have been approved, but their availability has been restricted, at least temporarily, through production and supply chain lags.

Mathematical models under specific objective functions have been used to determine the optimal balance between direct protection of the most vulnerable at risk individuals, or indirectly by vaccinating those who are most likely to transmit and infect others.

Models that have been used for calculating optimal balance between direct and indirect protection against the seasonal flu do not apply to COVID-19, which has several important and distinctive features, including an increasing number of escaped mutants that are difficult to incorporate in models under a range of conceivable assumptions.

Vaccine strategies for younger or older age cohorts depends on whether the number of infections or the number of deaths are to be prioritized.

Socially accepted non-vaccine measures include masking, personal hygiene, various types of social distancing that range from from self isolation to extended lockdowns, as well as treating 
mental health illness, accommodating socio-economic inequalities, and gender, race, and ethnic considerations, also need to be modelled under a range of alternative assumptions.

In short, as stated presciently in the perspective, age-specific considerations are not the most suitable for purposes of determining the most accurate and useful balance between the direct and indirect vaccine immunization strategies.

\section{COVID-19 Spillovers Between Species}

Zhou and Shi (2021) examine the COVID-19 spillovers between species.

SARS-CoV-2 that leads to the original wild-type COVID-19 disease, and any mutated strains that lead to possibly more highly infectious variations of the disease, such as those from UK, South Africa, and possibly Spain, have been investigated empirically, though not necessarily in clinical trials, especially as the mutations have only recently come to notice.

It is well known that various coronaviruses can be transmitted by bats, camels, pangolins, mink, domesticated animals such as cats and dogs, lions, tigers, and civets, that have come into close contact with infected mammals, especially humans.

The precise cause of SARS-CoV-2 is as yet indeterminate, though the COVID-19 pandemic is widely regarded as having started in Wuhan, China in late 2019.

Spillover infections of the virus are known to have been transmitted between mink and humans, starting in The Netherlands, Denmark, and the USA, among other countries.

Massive culling of animals in mink farms seems to have controlled the transmission and infection rates, but such culling is infeasible for all bats, camels, pangolins, domesticated cats and dogs, while the numbers of pangolins, civets, lions, and tigers, some of which are under threat of extinction, cannot be determined precisely.

Whether mutated strains, not all of which have yet been discovered, have the same propensity as the original wild-type virus for spillover effects between animals and humans has not yet been clinically tested.

A similar comment applies to the various approved vaccines to date.

\section{Comovements in Science and Policy During COVID-19}


An experienced and expert team of medical scientists in Yin, Gao, Jones and Wang (2021) have investigated the science and policy foundations regarding hospitalization, and the physical and mental medical and healthcare policy implications of COVID-19.

That science and associated public policy more together is not surprising, but which moves faster, and whether there are any bilateral mutual causality effect is not entirely clear.

In a world that has embraced "fake news" and heaped scorn on science, it is unclear whether the science and associated policy are being presented in the global news media and social media accurately, or are being understood clearly.

The data are based on 37,725 policy documents published by government agencies and think tanks from 114 countries and 55 IGOs, from 2 January - 26 May 2020, which is relatively early in the pandemic.

The second and higher waves of the pandemic that started in November 2020 may well have a different relationship between science and policy, especially in light of the 2020 Presidential Election in the USA, as well as the production and distribution of the various approved vaccines to date, and the recent discovery of the more highly infectious mutated strains of the SARS-CoV2 virus that causes the wild-type COVID-19 disease.

The public policy implications of COVID-19 are not only related to disciplines in medical science, but also the social science, economic, and finance implications in order to deal with the financial costs of the pandemic.

\section{Mathematical and Statistical Models to Guide Informed COVID-19 Responses}

The detailed and helpful explanation of mathematical models by Metcalf, Morris and Park (2020) makes it clear that the outcomes of mathematical modelling can help to guide sensible, directed, and timely responses to the ongoing COVID-19 pandemic.

The research perspective was published relatively early during the pandemic, and well before the second and higher waves of infection occurred, before escaped mutants and long haul COVID19 effects on purportedly recovered patients were discovered, and also before safe, effective, durable, affordable, and available vaccines were created.

Herd immunity is intended to protect the population through either a high threshold $\%$ of the population through infection or vaccination. 
The unknown threshold is a parameter that needs to be estimated, so a model with appropriate underlying assumptions and unknown parameters must be specified.

Robust inferences regarding the estimates and implications of the empirical analysis can be strengthened with the application of diagnostic checks to test the underlying assumptions of the models.

As additional time series data become available across a number of countries, more informative dynamic models and associated estimates can be used to guide how important aspects of the pandemic are changing over time, such as the likelihood of seasonal COVID-19, the optimal times between the first and second jabs of approved vaccines, and the likelihood of individuals being infected more than once.

The outcomes of clinical trials can guide public healthcare policy, such as the performance of the approved vaccines against the escaped mutants.

However, predicting future mutated strains and variants, and the likely performance of the vaccines, higher waves of the pandemic, the benefits of social mandates, including masking and whether lock downs work, among many others, require statistical models and an evaluation of their accuracy.

\section{Preclinical Efficacy and Targeting of SARS-CoV-2 and COVID-19}

The impressive research team in White, Rosales, Yildiz, Kehrer, Miorin, Moreno et al. (2021) reports on the preclinical efficacy through targeting the host protein.

It is found that the drug plitidepsin (aplidin), with limited clinical approval, possesses antiviral activity that is more potent than remdesivir against SARS-CoV-2 and COVID-19, with limited toxicity in cell culture and potent antiviral effects.

Whether the antiviral remdesivir, together with oxygen therapy and ventilation and the antiinflammatory dexamethasone, should be used as a benchmark might be of some concern, especially with the advent of a number of approved vaccines.

It is also reported that a host-targeted antiviral might offer greater protection from viral escaped mutants, such as the UK variant or strain, than viral-targeted therapeutics and vaccines.

There was no mention of the mutated viruses from South Africa and Brazil, and possibly also from the USA, specifically California at this stage. 
Although the experiments were performed on mice, the results look promising for future clinical trials on humans, especially if COVID-19 continues unabated for an extended period, as well as for future outbreaks of possibly more infectious coronaviruses.

\section{Engineering Potent Antibodies Against SARS-CoV-2 and COVID-19}

The impressive research team in Rappazzo, Tse, Kaku, Wrapp, Sakharkar, Huang et al. (2021) investigated broad and potent activity through neutralization against SARS-like viruses, including SARS-CoV-2, by an engineered human monoclonal antibody.

Although the study was based on immunocompetent mouse models, the results suggest a promising therapeutic treatment for protective antibodies alongside the currently approved vaccines.

Moreover, the antibodies were reported as retaining high affinity binding to the rapidly spreading UK escaped mutated strain, which suggests that the antibodies should be capable of neutralizing a range of emerging coronaviruses.

Whether such antibodies will retain potency against the mutated strains and variants from

South Africa and Brazil, as well as from the USA, meaning predominantly California, remains to be seen.

As the currently approved vaccines that are being distributed globally have not yet been clinically tested against the emerging mutated strains, it would also be worth examining if engineering antibodies might be used in potentially more potent vaccines.

\section{Viral Mutants Escaping Antibodies for Treating COVID-19}

An impressive team of medical experts in Starr, Greaney, Addetia, Hannon, Choudhary et al. (2021) report on the prospective mapping of viral mutations that escape antibodies for treating COVID-19.

Antibodies can be therapeutic for the SARS-CoV-2 virus that causes COVID-19, but the virus can escape and evolve into mutated strains and variants.

Mapping the movements and trajectories of the original wild-type SARS-CoV-2 and the mutated strains can be used to identify the escaped mutants, which may be present in the mutated 
strains that are presently in circulation, such as those from the UK, South Africa, Brazil, and the USA, predominantly in California.

In addition to aiding interpretation and analysis, complete escape maps might also be able to predict how the mutated strains evolve, whether the range of approved vaccines that are presently being distributed globally will be safe, effective, and durable against the mutated strains, and whether the virus and its mutated variants evolve to develop widespread resistance to the antibodies.

Examining antibodies to map the original virus and its mutated variants may provide a range of options for medical researchers and healthcare professionals in anticipating the range of PPE that might be necessary to treat patients infected with possibly unknown mutations.

Such informative mapping would also be useful for vaccine producers to incorporate the knowledge regarding mutant strains in developing safer, and more effective and durable vaccines.

\section{Can China Help to Alleviate Bilateral Debt Payments During COVID-19?}

As mentioned in Simmons, Ray, Yang and Gallagher (2021), there is no question that COVID19 has led to disastrous consequences for global health, and the associated economic crisis, including external debt distress.

Suspending bilateral debt payments, including interest, for low income countries has benefits in the short run, but payments must eventually be made, either by the G20 countries or by the low income countries themselves

If payments are not possible, especially in the time of COVID-19, suspension of debt payments will need to be replaced by debt cancellation, at least in part

Regardless of whether China and the associated government banks are the largest bilateral creditors, especially for the low income countries, can China be relied upon to alleviate debt payments for altruistic reasons, especially when the large policy banks in China are not coordinated, and when many low income countries have also borrowed from private lenders?

\section{Pricing Allowances to Mitigate Carbon Dioxide Emissions During COVID-19}

Voosen (2021) examines the legacy of Donald Trump, who downplayed the cost of carbon. 
Carbon dioxide (CO2) emissions and greenhouse gases are having a serious and negative effect on global warming and climate change, which affects everyone globally.

Without concrete steps to mitigate the effects of $(\mathrm{CO} 2)$, the effects on the global environment, healthcare, and economy, among others, will only worsen.

Many countries have had to curtail their efforts to counter $\mathrm{CO} 2$ emissions because of the insurmountable costs of dealing with the COVID-19 pandemic.

Effectively ignoring one global problem to deal with another makes it possible for $\mathrm{CO} 2$ emissions to have a mounting effect on the global community, including increasing healthcare costs and distress, including shortness of breath and chest pain, among a host of respiratory problems.

An encouraging attempt to mitigate $\mathrm{CO} 2$ emissions involves the use of carbon allowances that permit companies, industry sectors, geographic regions, and countries that participate in mandated national, or preferably international, carbon markets to emit $\mathrm{CO} 2$, at a financial cost that is regulated by the market mechanism.

The European Union, parts of the USA, predominantly California and, more recently, China are currently involved in pricing carbon emission allowances to restrict the amount of greenhouse gas that enters the atmosphere.

Although such attempts are being developed at the national level, negotiations for extending carbon allowances to the international level need to be advanced more swiftly to mitigate $\mathrm{CO} 2$ emissions, especially during COVID-19.

\section{Sequencing SARS-CoV-2 RNA Genomes to Mitigate COVID-19}

The medical experts in Martin, VanInsberghe and Koelle (2021) report successful attempts to sequence SARS-CoV-2 RNA genomes to understand and mitigate the spread of COVID-19, both spatially and inter-temporally

A critical analysis of the emergence of escaped mutants of the original wild-type coronavirus is essential in order to ensure that vaccination programs are likely to lead to herd immunity through

protecting a high proportion of the population, rather than relying on infecting a high proportion of the population. 
The perspective states: "it is not clear whether more virulent SARS-CoV-2 strains are likely to evolve."

This seems to ignore the strains and variants of COVID-19 that have already evolved and been

discovered in the UK, South Africa, Brazil, and the USA, predominantly in California, which can be more transmissible and infectious than the original strain.

Sequencing can also assist in contact tracing, travel history mapping, and genomic epidemiology, which are essential to restrict the spread of the disease as infected individuals mix with others in the community, both domestically and internationally.

These developments will assist public healthcare officials to develop policy strategies to deal most efficiently with the pandemic.

\section{Unanswered Questions About Antiviral Immunity to COVID-19}

As can be discerned from the expertise in Hope and Bradley (2021), there is much to learn, and possibly even more that remains unknown, about COVID-19, including highly variable immune responses and divergent clinical outcomes concerning the SARS-CoV-2 virus.

The duration of immunological memory and lasting protection from being infected by the virus are among a host of unanswered questions including, especially for cancer patients and other vulnerable individuals with existing comorbidities:

(1) Is it safe to be vaccinated against COVID-19?

(2) Should there be prioritization or deprioritization for vaccination?

(3) Is vaccination against COVID-19 regarded as aggressive critical care?

(4) Should residents in palliative care be vaccinated?

(5) Does vaccination add to the quality of life?

(6) What are the effects of mutated strains and variants of the original wild type coronavirus?

(7) Should nonpharmaceutical interventions be considered in critical care?

(8) What are the effects of mild, medium or severe COVID-19?

(9) Should all types of cancer be considered in combination with vaccination?

(10) Is the cancer primary or secondary?

(11) What are the likely additional long haul effects from COVID-19? 
Should mental healthcare for COVID-19 be expanded?

(13) What are the effects of the second and higher waves of COVID-19 infections?

(14) Should overlapping responsibilities between the federal and state governments for COVID-19 be extended?

(15) How will healthcare access, hospitalization, and healthcare insurance premiums change to cover and protect against COVID-19 and a host of other diseases?

\section{Neutralization of Antibodies Against Escaped Mutants}

It is known that there have been escaped mutants of the original wild-type coronavirus (COVID-19) in the UK, South Africa, Brazil, and possibly also the USA, that are more highly transmissible and infectious.

The UK is presently grappling with both the home-grown mutated strain, as well as the on from South Africa.

As discussed in Muik, Wallisch, Sänger, Swanson, Mühl, Chen, Cai et al. (2021), the neutralizing antibodies may fail to recognize the UK and other variants because of the associated large number of amino acid changes, so human immune sera were used to restrict the mutations while preserving the biological neutralization.

As young and older adults were tested, and found not to be different statistically, using a wider range of age cohorts and gender against mild, medium, and severe mutated variants would be informative.

Whether the neutralization would be effective as mediated protection against the other untested strains remains to be seen.

\section{Adapting Vaccines to Escaped Mutants}

The informative report in Kupferschmidt (2021c) leads to questions such as how and when the producers of approved vaccines should adapt their vaccines to mutated strains and variants of the original wild-type coronavirus (COVID-19) is a matter of topical and extreme importance, given the escaped mutants that have already arisen in the UK, South Africa, Brazil, and possibly the USA (specifically in California). 
It is all the more urgent, as the UK is presently experiencing a combination of the domestic variant, as well as the one from South Africa.

Issues such as the safety, efficacy, and duration of the existing vaccines to some or all of the mutated strains and variants have a significant impact on public healthcare and safety, as well as possibly affecting the willingness of individuals to be vaccinated.

The number of mutated strains and variants seems to be positively correlated with the number of infections, so restricting the spread and replication of COVID-19 through vaccinations is critical to the safety, effectiveness, and duration of existing vaccines, their potency in inducing neutralizing antibodies, and how fast immunity wears off after vaccination.

Unless existing vaccines can be adapted to the escaped mutants, which have been known top evade immunity, the global distribution of vaccines will not necessarily lead to herd immunity, where a high (> 80\%) proportion of the population in each country and territory needs to be vaccinated, or be infected and recover.

\section{Public Health and Social Measures During COVID-19 Vaccinations}

The medical and public health experts in Galanti, Pei, Yamana, Angulo, Charos, Swerdlow and Shaman (2021), recommend that social distancing should be maintained during vaccinations for COVID-19.

The broader public health and social measures include wearing masks, maintaining personal hygiene, and non-pharmaceutical interventions (NPIs), among others, to limit the spread of the disease.

The two-shot vaccines require 21 days between jabs, which is more than enough time for individuals to be infected before the vaccine reaches full effectiveness.

As vaccination of children and adolescents has not yet been approved, and it is not yet known with clarity whether children are more or less likely to become infected, transmit and infect others, or might be protected through vaccination, a cautious approach is essential.

For herd immunity to occur, a high proportion of the population, which is widely regarded as a threshold greater than $80 \%$, needs to be vaccinated, or recover from viral infection. 
As the former case is more likely to occur, public health and social measures need to be respected, accepted, maintained, and become routine, as well as compliance with NPIs, before the threshold is reached.

\section{Interpreting the Outcomes of COVID-19 Tests}

The expert team of researchers in Robinson-McCarthy, Mijalis, Filsinger, de Puig, Donghia, Schaus et al. (2021) examine the anomalous outcomes of COVID-19 tests.

Correct outcomes can only be determined from clinical trials where volunteers are monitored against confirmed infections and placebos.

In the real world, there can be false positives and false negatives, each with associated social consequences, especially arising from nasal swabs as compared with saliva samples, which can lead to mixed results.

Asymptomatic medical researchers working with non-infectious SARS-CoV-2 nucleic acids can be detected with anomalous surveillance test results, so that further testing is required to determine if there is a true active infection.

Waste water checks frequently lead to false positive outcomes, even without an outbreak of infections in the locality.

These mixed results suggest that caution should be exercised before determining the outcome of tests for the presence of COVID-19.

\section{Disinfection Against COVID-19 and Antimicrobial Resistance}

The informative report by wastewater management experts in Lu and Guo (2021) examines

disinfection by individuals in personal hygiene, and public authorities in managing wastewater treatment through increased chlorine dosage.

There is a balance that needs to be weighed between mitigating the spread of COVID-19 and excessive disinfection that could increase antimicrobial resistance, despite the intention being to inactivate the human coronavirus and associated disease.

Through human interaction, food, water, and air, the environment can act as a conduit for the spread of COVID-19, especially if antimicrobial resistance is accelerated. 
The environment as a potential source of spreading COVID-19 over an extended period highlights the need to acknowledge environmental degradation, including global warming, climate change, and dechlorinating the treated effluent before discharging as wastewater, as essential elements in accommodating and managing public health risk and safety in the era of COVID-19.

\section{Augmenting Antiviral Immune Responses of Vaccines for COVID-19}

The expert medical researchers in Juno and O'Connor (2021) examine the translation of viral vaccines into immunity, which might be applied to augmenting antiviral immune responses of vaccines for COVID-19.

Vaccine-mediated induction may reflect vaccine immunogenicity, but the relevance of such strategies might be evaluated in the context of:

(1) the suitability of such viral vaccine translations for humans;

(2) the safety, effectiveness, and duration of such viral vaccines against the original wild-type coronavirus (COVID-19), as well as against the highly transmissible and infectious escaped mutants from the UK, South Africa, Brazil, and the USA, among possibly others;

(3) how the protective immunity might be evaluated according to gender and age cohorts, especially children and adolescents versus the aged;

(4) how the protective and adaptive immunity might be evaluated according to preexisting comorbidities and immunocompromised individuals;

(5) the potency of adaptive antiviral immunity for vaccines against COVID-19;

(6) whether augmenting the antiviral immune responses of vaccines might mitigate the effects of long haul recovery effects from COVID-19 through long-lasting protective immunity.

\section{Age Demographics Propelling Higher Waves of COVID-19}

An impressive team of medical researchers in Monod, Blenkinsop, Xi, Hebert, Bershan, Tietze et al. (2021) examine the age groups and demographics that have sustained the global resurgence of COVID-19, with an emphasis on the USA. 
Whether the age demographics are driving transmission, and how they might be associated with the loosening of interventions, including transmission-blocking vaccines, is an empirical issue where the direction of causation should be tested using formal statistical methods.

The research article presumes that, in the context of age-specific COVID-19 mortality data, the direction of causality is uni-directional, from the age demographics to transmission, and from transmission to the loosening of interventions, when both relationships could be bi-directional, with the causation moving in both directions.

The empirical analysis finds that individuals in only the 20-49 years age cohort are observed to sustain resurgent SARS-CoV-2 transmission, and recommend that adults in this age cohort should be targeted with interventions, including mass vaccination with transmission-blocking vaccines to control the higher waves of COVID-19.

As detailed longitudinal human contact and mobility data are difficult to determine for purposes of determining the transmissions mechanisms, statistical models with strong underlying assumptions are crucial in determining the relationships among the age demographics, transmission, and the loosening of interventions.

The data were based on cell-phone data to capture rebounds in mobility across age-specific groups, COVID-19 death time series were reconstructed from cumulative counts for model estimation, where it was shown there were no statistical changes in intertemporal age-specific disease dynamics.

Estimates from one stage were used in subsequent stages, which leads to biases in the generated or estimated quantities.

The absence of regression diagnostic checks makes it difficult to determine the overall robustness of the empirical results in an impressive research report.

Compounding the higher waves of the COVID-19 pandemic have been the discovery, production, and distribution of a variety of approved, safe and effective vaccines of as yet unknown duration, as well as the emergence of escaped mutants from the UK, South Africa, Brazil, and possibly the USA, against which the safety and efficacy of the existing vaccines are presently unknown. 


\section{Escaping Antibodies, Recurrent Deletions, and Evading Immunity from SARS-CoV- 2 and COVID-19}

The team of experts in McCarthy, Rennick, Nambulli, Robinson-McCarthy, Bain et al. (2021) report on how recurrent deletions in the SARS-CoV-2 spike glycoprotein, which cannot be corrected, drive antibody escape and accelerate adaptive viral evolution.

This is essential reading for the current COVID-19 pandemic as it is reported that recurrent deletions are associated with accelerating SARS-CoV-2 antigenic evolution which, in turn, is likely to drive adaptive evolution.

Such a causal relationship would go a long way toward explaining the currently increasing number of escaped mutants observed in the UK, South Africa, Brazil, and the USA, among others.

Humoral immunity is associated with protection, and has been incorporated in several recently approved safe, effective, possibly durable, vaccines that are presently being produced and distributed globally to provide immunity with the goal of achieving herd immunity.

Data were collected from an immunocompromised cancer patient infected with COVID-19, who succumbed, was consensus sequenced for cloned genes in order to identify two variants with deletions.

Subsequent interrogating of medical data led to the identification of eight other patients with similar deletion and duration characteristics.

Evaluation of the genetic spatial and temporal sampling of variants suggested they have been present throughout the pandemic, and are likely to continue mutating at regular temporal intervals, which is both informative and troubling, especially for long-term infections and immunocompromised individuals.

As deleted variants resist neutralization by monoclonal antibodies, it is essential to conduct clinical laboratory trials to determine if the presently approved vaccines wit]ll be safe, effective, and durable against the known mutated and deleted viral strains and variants, as well as those that might presently be unknown but might be predicted through appropriate mapping of recurrent and convergent patterns of adaptation.

\section{Escaped Mutants and Herd Immunity}


Herd immunity can be achieved during a pandemic when a sufficiently high proportion (in truth, the threshold is unknown, but it is widely regarded as greater than 80\%)of the population recovers after infection, or if a similarly high proportion is vaccinated against COVID-19.

As discussed in Kupferschmidt (2021d), this presumes that infection and vaccines are dealing with a specific virus, such as SARS-CoV-2, that leads to a specific disease, such as COVID-19.

The assumptions underlying a standard herd immunity strategy are insufficient in the presence of mutated strains and variants, such as those that have been discovered in the UK, South Africa, Brazil, and possibly the USA, among others.

The approved vaccines have been shown to be less effective, and of unknown duration, against the escaped mutants, which lead to greater transmission and infections, with an as yet unknown effect on the number of deaths.

The effects of the escaped mutants can be made more destructive if there are interactions among them, or if they spread simultaneously in the population.

The unknown optimal timing between the first and second doses of approved vaccines, whether it be 21 or 28 days, or possibly several months, makes it possible for a virus to mutate further and more frequently.

Moreover, if several mutated strains and variants spread in the community, possibly together with the original wild-type coronavirus, it might be necessary to be protected using more than one type of vaccine.

It is essential for global healthcare in trying to mitigate the effects of the pandemic to map the mutated strains and variants that are most likely to mutate even further and more frequently, and to finesse the existing vaccines to be safe, effective, durable, affordable, and available to protect the global population, and before any existing immunity begins to wane and render reinfection more common.

\section{New Vaccines for Mild to Severe Symptoms from COVID-19}

As evaluated in Cohen (2021a), any vaccine for COVID-19 that is safe, efficacious, durable, affordable, widely available, and prevents recurrence, especially in the short run, will be welcomed by the global community and healthcare providers at a time of great need, especially for mild and medium symptoms. 
Production and distribution of some approved vaccines can be troubled by bottle necked supply chains, especially in countries which are less efficient technologically in terms of advanced healthcare facilities and medical treatment, including vaccines that have to be stored at very low temperatures.

Evolving mutant strains and variants of the original wild-type coronavirus might render less effective and potent the antibodies that are produced through viral infection or immunity induced through vaccination.

Reducing severe symptoms of COVID-19, such as hospitalizations and deaths, is a critically important aspect of any approved vaccines, but their performance against the emerging mutant strains and variants needs to be evaluated through clinical laboratory trials, rather than relying on the PR and media announcements that are optimistically promoted and distributed by their producers, frequently without peer review.

Reducing the number of jabs from two to one, which will eliminate the possibility of infection between the two jabs at a delayed timing of 21 or 28 days especially for vaccines that can also be stored at well above freezing temperature, would also instill optimism and confidence to all members of society.

As COVID-19 is now the Number 1 cause of death in many countries worldwide, any vaccine that mitigates against the most severe symptoms arising from the disease, regardless of age, gender, and preexisting immunodeficiencies, is a gift of insurmountable dimension.

\section{Escaped Mutants of SARS-CoV-2 and COVID-19}

Good and bad news seem to go hand in hand, as in the case of the rapidly shrinking infections and spread of the original wild-type coronavirus (COVID-19) and its replacement in many countries by several escaped mutants from the UK, South Africa, Brazil, and possibly the USA.

The informative report in Kupferschmidt (2021e) pays close attention to Denmark to evaluate whether penetrating lessons can be discerned from the explosion of a specific viral strain or variant in the UK and Ireland.

Whether the existing approved vaccines will be less potent against the escaped mutants is of considerable import to medical researchers, as is the possible progression from mild to severe viral 
infections, as well as the possibility of experiencing long hail COVID arising from the original wild-type and the escaped mutants.

Regardless of what some experts might think, SARS-CoV-2 is not remotely like the seasonal flu in terms of its known escaped mutants and long haul COVID effects, to which the failed Swedish experiment in the difficult task of achieving herd immunity would attest.

Incidentally, in addition to New Zealand, Australia, Taiwan, Vietnam, and a number of isolated Pacific island nations and territories with small populations have relied quite successfully on strict border lockdowns to try to eliminate the disease.

\section{Are Vaccines Safe, Effective, and Durable Against Escaped Mutants?}

Although the prescient report in Cohen (2020b) was published two months ago, and before substantial information was known about the number, transmission, infectious, and contagious nature of escaped mutants, as well as the availability of approved vaccines against the original wild-type coronavirus (COVID-19), the leading question remains topical and important for healthcare public policy amid the spread of the pandemic.

In short, are vaccines safe, effective, and durable against escaped mutants, such as those discovered in the UK, South Africa, Spain, Brazil, and possibly the USA?

As COVID-19 is now the Number 1 cause of death in many countries worldwide, and the mutated strains and variants are increasing exponentially and replacing the original coronavirus, are the approved vaccines likely to be effective in providing protection against any, some, combination, or all of the mutations?

All vaccines are likely to lose some potency against strains and variants for which they were not specifically targeted, but Moderna and Pfizer are said to be around 95\% effective, so that a reduction in their efficacy would render them as viable vaccines.

The distribution of the AstraZeneca/Oxford vaccine was halted within the past few days when it was found to have low efficacy against the South African mutation which, on the face of the empirical evidence, seems counter intuitive in that it has been reported as being potent against severe COVID-19.

The important question that remains to be answered is the difference between severe COVID19 and the various mutated strains and variants. 
An additional query relates to the ease with which vaccines can be shipped, distributed, and stored at the required temperatures in areas where refrigeration facilities and energy sources may be questionable or problematic.

As most existing approved vaccines require two jabs, the prime and booster shots, either at 21 or 28 days apart for Pfizer and Moderna, respectively, and from one month to12 weeks for AstraZeneca, what is the optimal duration between jabs, and what is the interval beyond which the second jab begins to lose potency?

\section{Ethnic Diversity in Clinical Trials for Better COVID-19 Vaccines}

As argued forcefully in Bumpus (2021), the lack of ethnic diversity in clinical trials globally refers to the disproportionately low representation of Black, Latinx, Indigenous people, and individuals of non-European descent, who are most heavily affected by healthcare coverage and diseases.

Whether such disparities are intentional or not, the informative perspective argues convincingly that it has a negative effect on the quality of drugs as the lack of variation across the population leads to outcomes in reference to medical and therapeutic treatments that do not represent the population.

This is all the more telling and topical when vaccines are being distributed across the population globally, based on clinical trials that do not represent the population, with exclusions of young children and adolescents, the aged, ethnic minorities, pregnant women, individuals with existing comorbidities, and omissions based on socio-economic considerations, among others.

Vaccines must be based on safe dosages, effective for mild, medium, and severe infections, durable, affordable, and widely distributed, which refers to everyone in the population, but clinical trials do not capture a representative sample of the population.

This is compounded by the emergence of more transmissible, infectious, and contagious mutated strains and variants of the original wild-type coronavirus (COVID-19), which tend to reduce the potency of the approved vaccines.

As the effects of vaccines can have significantly different effects across ethnic groups, with different genetic variations, such as a higher risk of major bleeding widely prescribed 
anticoagulants, as well as respiratory distress, ethnic diversity is essential in clinical trials, especially for the SARS-CoV-2 virus that seems to discriminate against many excluded individuals.

The strong recommendation to report the preferred proportional representation of the population in clinical trials for testing vaccines that will ultimately be distributed to everyone globally, is an excellent requirement for all future clinical trials.

\section{Contact Tracing for COVID-19 Helps Everyone}

As presented in an early career story in Naseer and Strelau (2021), it is heartwarming to see how working from home and away from the office and lab can benefit everyone involved in a public health, social work and medically-related project that involves contact tracing for individuals who had tested positive for COVID-19.

Undertaking research for a PhD degree can present many difficulties, both expected and unexpected, but the discipline and mental fortitude required to spend several years on one or more research chapters or papers can help to develop specific skill sets.

Conducting best practice contact tracing requires substantial financial and human resources, and is usually conducted at the city, state, or regional level.

Speed is of the essence, especially as contacts might no longer be available for tracing.

If these talented organizers can handle months of mental stress associated with conducting and managing contact tracing, completing their $\mathrm{PhD}$ research should be a breeze.

\section{Science Metric Initiatives}

As in all the prescient editorials (see Thorp (2021b)), monitoring change through initiatives on leading metrics is a significant step forward in the context of informational and scientific records regarding the six main journals in the Science portfolio.

The acceptance rates for Science, Science Immunology, and Science Translational Medicine are very low at around 6\%, while the submission to acceptance times are the highest for Science Signaling and Science Translational Medicine. 
The timing distributions of Citations are similar for five of the six journals, all of which reach their respective maxima in the first ten days, with Science reaching its maximum in the second ten days.

The range of journal metrics includes the Web of Science 2-year and 5-year impact factors, immediacy index, eigenvalue score, article influence score (which is misnamed as it is the mean eigenfactor score, and refers to a journal rather than it articles), h-index, and CiteScore (namely, the Scopus 4-year impact factor).

All the journal metrics are consistent with each other, with Science the leader in each category, followed by Science Robotics and Science Translational Medicine, and distantly by Science Signaling.

The detailed information on leading metrics broadens the information set that is of considerable value for the academic and professional editors of journals, and all academics who use such information to determine which journals might warrant their submissions.

\section{Viral Sequencing in Detecting COVID-19 Strains and Variants}

As discussed in Wadman (2021a), the failure to engage in substantial viral sequencing has made it difficult to detect the emerging strains and variants in SARS-CoV-2 and COVID-19 in the USA that have significant impacts on the safety, efficacy, and duration of the currently approved vaccines.

Without viral sequencing of at least 5\% of confirmed COVID-19 cases in each State, leading scientists have warned that mutated strains and variants, such as those from the UK, South Africa, Brazil, and possibly Spain and California, will be difficult to anticipate and detect.

The availability of sequencing scientists and facilities is not an issue, but the mismanagement in coordinating a national surveillance system is a serious issue.

This is all the more troubling when the discovery of more highly transmissible, infectious, contagious, virulent, and lethal escaped mutants can reduce the potency of currently approved vaccines.

Predicting the dominance and durability of specific viral variants, as well as their geographic and spatial locations, would be of immense assistance to public healthcare authorities, but this cannot be done without appropriate viral sequencing. 
In States where the viral sequencing proportions of confirmed COVID-19 cases are the lowest, it would be virtually impossible to detect the emergence and spread of viral mutants, and to prepare public healthcare officials and facilities to deal with the associated infections.

\section{Single-domain Antibodies that Neutralize Replication of SARS-CoV-2 and COVID-} 19

Saelens and Schepens (2021) discuss combinations of single-domain antibodies that neutralize replication of SARS-CoV-2 and COVID-19 have been proposed as potential therapies for the treatment of COVID-19 patients

It remains to be seen whether single-domain antibodies can be effective in dealing with the emerging mutated strains and variants of the original wild-type coronavirus (COVID-19), such as those in the UK, South Africa, Brazil, and possibly Spain and California

Whether the presently approved vaccines can be made more potent against the escaped mutants based on a range of possible combinations of single-domain antibodies is also of significant public policy interest and practice

Clinical trials seem to be in process, but the range of mutated strains and variants, the choice of finely tuned vaccines, alternative combinations of single-domain antibodies, and the selection of a sufficiently large number of trial patients, would need to be extensive to lead to potentially encouraging outcomes in neutralizing viral replications to protect individual against infection.

\section{Tracing SARS-CoV-2 and COVID-19 in the UK}

The interesting and informative report in Nelson (2021) on tracking the UK SARS-CoV-2 outbreak is timely and important as the UK is one of the most heavily infected countries with the original wild-type strain of coronavirus (COVID-19), as well as the home-grown mutated strain and variant, together with the emergence of the escaped mutant from South Africa.

Innovative research that investigates viral sequencing in detecting COVID-19 strains, variants, and lineages, single-domain antibodies that neutralize replication of SARS-CoV-2 and COVID19, genetic tracing, and tracking escaped mutants, among others, can assist in tracking the virus and disease in the UK. 
Strict border controls for both national and international air travel, tracking human movements carefully, precise contact tracing, and following a wide range of social conventions, are essential in tracing rapid and volatile movements in the spread of the disease.

The discovery of emerging mutated strains and variants, such as from Brazil and California, can complicate the environment, especially when more than one variant arises contemporaneously and spatially.

The safety, efficacy, duration, and potency of currently approved vaccines in the face of emerging mutations are also important considerations that need to be clinically tested and observed empirically in practice.

\section{COVID-19 Penalty for Academic Mothers}

If data from the NBER (2021) suggest that there is a "motherhood penalty" arising from the COVID-19 pandemic for academic mothers, it can be taken as accurate, as proposed in Langin (2021).

It is well known that, regardless of country, culture, ethnicity, employment, and socioeconomic considerations, among others, mothers have traditionally borne a disproportionately heavy burden of child rearing and domestic household duties.

If research and the associated funding opportunities and access continue to be disproportionately allocated by gender, this cannot represent the optimal use of academic talent anywhere.

The COVID-19 pandemic should be a period of addressing inequities rather than imposing a "motherhood penalty" on already overworked academic mothers.

\section{A Balanced Life During COVID-19}

The heartfelt working life career stories raise many personal issues that are specific to some, and common to many.

As told in Fujiwara (2021), the prescient and penetrating advice of a doctor with impeccable bedside manners is a joy to behold. 
Enjoying life is a goal toward achieving happiness, as well as the meaning of life, at least to many.

Apart from drug addiction, prescription sleeping pills should be close to a last resort as they do not target whatever might be the cause of the illness, as distinct from the observed effects.

"A change is as good as a rest" is a phrase that captures the feeling that a change from a regular occupation is as beneficial as a relaxing holiday.

A simple and balanced life can cure many perceived and real illnesses, especially during the COVID-19 era.

\section{Lipopeptides, Intranasal Prophylaxis, and SARS-CoV-2 Transmission}

The innovative research report by a wide-ranging team of medical experts in de Vries, Schmitz, Bovier, Predella, Khao et al. (2021) on the administration of lipopeptides that are designed to translate intranasal prophylaxis to reduce and prevent SARS-CoV-2 transmission through direct contact or aerosol transmission.

Although the initial experiments were conducted on ferrets, the experimental outcomes that provided complete protection during a 24-hour period of intense direct contact would seem to open promising areas of research that could lead to protect humans against the first step in the COVID19 infection chain.

While the validity and potency of the approved vaccines against the original wild-type coronavirus (COVID-19) and the numerous emerging strains and variants from the UK, South Africa, Brazil, and the USA were not discussed in detail, a potent lipopeptide robustly inhibited fusion by their respective spike glycoproteins (or $\mathrm{S}$ proteins).

In the process of protecting the respiratory tract, the authors proposed a SARS-CoV-2 specific lipopeptide for intranasal prophylaxis against transmission of the virus and disease in humans.

It would be informative to test whether such potency against transmission were to remain in the presence of a contemporaneous emergence of more than one mutated strain or variant.

Moreover, moderating the innovative experimental outcomes for mild, medium, and severe forms of COVID-19 might also suggest how intranasal prophylaxis to reduce and prevent SARSCoV-2 transmission might be extended to direct contact or aerosol transmission among humans, excluding transmission via orofecal, scratching, or biting, as in the case of ferrets. 


\section{Impaired Exhaustion and Severe COVID-19}

As the molecular properties of $\mathrm{T}$ cells that respond to SARS-CoV-2 infection are not fully known, an impressive team of medical researchers in Kusnadi, Ramírez-Suástegui, Fajardo, Chee, Meckiff et al. (2021) report informative research on impaired exhaustion with different clinical severity, emphasizing 39 COVID-19 patients, varying from 17 patients with mild infections, 13 hospitalized patients, 9 patients requiring ICU support, and 10 healthy non-exposed individuals.

Based on single-cell analysis, and a detailed biostatistical analysis of exhaustion-related markers in global T cell populations, and comparisons of exhausted and non-exhausted clusters, it was discovered that there was substantial diversity in the nature of $\mathrm{T}$ cells responding to SARSCoV-2.

Approved vaccines are intended to provide safe, effective, and durable protection against COVID-19 and reduce severe reactions, including death, analyzing connections of paired exhaustion with the emerging mutated strains and variants from the UK, South Africa, Brazil, and the USA, among others, would be helpful to public healthcare policy providers and policy makers.

It was also found that exhausted SARS-CoV-2-reactive $\mathrm{T}$ cells, which are important in preventing excessive host tissue damage in viral infections, were increased for patients with mild COVID-19 illness, while pro-survival features were present in $\mathrm{T}$ cells for patients with severe COVID-19.

However, it was not determined if SARS-CoV-2 infection generated long-term memory T cells because of the short time interval between the onset of COVID-219 symptoms and blood collection for empirical evaluation.

The absence of a variety of diagnostic checks of the underlying model assumptions make it difficult to determine the robustness of the wide-ranging and impressive empirical analysis.

\section{Would a Universal COVID-19 Vaccine be Safe, Effective, and Durable?}

The informative and challenging editorial in Koff and Berkley (2021) begs the question of whether a universal COVID-19 vaccine be safe, effective, and durable, especially in light of the emergence of mutated strains and variants in the UK, South Africa, Brazil, and the USA. 
The strong and encouraging editorial begins with: "Although this pandemic is far from over, we now have the tools to end it, with the largest and most rapid global deployment of vaccines under way."

Not only is such a bold statement reassuring, but the emergence of more highly transmissible, infectious, contagious, virulent and deadly escaped mutants suggests that a universal COVID-19 vaccine is warranted, especially if the currently approved vaccines have reduced potency against the mutations.

There is annoying research on improving the coverage of existing vaccines, as well as mixing and matching a variety of approved vaccines, and only time will tell, whether a prime and booster strategy, with varying temporal durations between the two jabs, single dose vaccines, or combinations thereof, would approach a universal COVID-19 vaccine.

Modern technology may well empower and enable the development of universal vaccines as "scientifically feasible" to mitigate the spread of the pandemic, and possibly also "to end it" with safety and effectiveness mentioned, though not durability and the other queries raised above.

It is always much cheaper economically and financially, as well as more manageable in terms of healthcare access and social cohesion, to prevent rather than cure viruses and their associated diseases.

As the prevention of unknown and unanticipated pandemics is rarely predicted, including the likelihood of jumping species at any time in the future, this leads to severely unsettling and disastrous effects on the global community, as reflected in the COVID-19 pandemic.

\section{Main Protease of SARS-CoV-2 Antivirals Inhibit Replication}

A large research team in Qiao, Li, Zeng, Liu, Luo, Huang et al. (2021) have reported invaluable research results regarding the main protease of SARS-CoV-2 antiviral activity that inhibit viral replication, especially in lung viral loads and lesions, when experimented on mice using a transgenic mouse model.

Antiviral agents are able to fight against infection by SARS-CoV-2 and to mitigate the COVID-19 pandemic, so the development of antiviral drugs, which are limited in number at present, is essential. 
Although there are two viral proteases, the main protease is the closest to human protease, so it would seem to be the appropriate antiviral for the development of drugs to inhibit the spread of the virus and disease, as well as to guard against possible side effects.

The innovative experiments included infection data in an animal model to examine the effectiveness of antiviral activity in mice.

The exhaustive experimental trials did not seem to examine the inhibiting effects on viral replication arising from the emerging mutated strains and variants from the UK, South Africa, Brazil, and the USA, which might reduce the effectiveness of the antiviral activity on mice.

As there are many infections of human patients globally, with one of the most obvious symptoms being stress on lung viral loads and lung lesions, clinical trials on humans should be the focus of future experimental research projects that are needed urgently.

\section{Temporal Duration from COVID-19 to Pre-COVID-19 Normal}

The interesting and informative feature report by an informed writer in Cohen (2021b) on the likely long road back from COVID-19 normal to pre-COVID-19 normal, should it ever happen, rests on many changes in the global society, some of which will be harder to achieve than others.

The feature tackles several of the essential and urgent targets that need to be met, including the following:

(1) global distribution of safe, effective, and (an as yet unknown) durable approved vaccines;

(2) protection from spreading the disease after vaccination, especially for the aged, and individuals in aged-care facilities and nursing homes;

(3) extensive clinical trials for the possibly reduced potency of the vaccines against the emerging mutated strains and variants from the UK, South Africa, Brazil, and the USA, that seem to be more highly transmissible, infectious, contagious, and dangerous than the original wild-type coronavirus (COVID-19);

(4) observing the impact of global vaccinations on the spread of infections, and the impact on the numbers of infections, speed of infections, hospitalization, admission to ICUs, and mortality rates;

(5) the potency of the alternative approved vaccines for mild, medium, and severe infections; 
(6) the optimal temporal duration between the prime and booster shots for the two-shot Pfizer vaccine (at 21 days), Moderna vaccine (at 28 days), and AstraZeneca/OxfordUni vaccine (between one and three months);

(7) the potency of the single jab Johnson \& Johnson vaccine, against mild, medium, and severe disease;

(8) whether infection is possible or likely between the prime and booster shots, and whether the temporal duration can affect the possibility and speed of recurrence;

(9) flexibility in the interval between and range of the prime and booster shots; combinations among any or all of the available vaccines in the development of a universal vaccine against COVID-19.

One of the main reasons for vaccinating a sufficiently high, though unknown, $\%$ of the population is to achieve herd immunity, whereby there are few unprotected individuals to continue spreading the disease.

An alternative strategy to achieve herd immunity is to allow a sufficiently high, and again unknown, $\%$ of the population to be infected and to purportedly recover, at least in the short term without exhibiting long haul COVID-19 symptoms that can arise in mild, medium and severe COVID-19 infections.

A serious problem with the herd immunity strategy is that it applies to a particular strain or variant of the coronavirus, with protection through vaccination or infection and recovery not guaranteed against the emerging escaped mutants, which is a disturbing prospect.

\section{Mentoring and Supervision Are More Important than the Research Topic, Especially During COVID-19}

The prescient, personal, and informative career stories, such as by Wengert (2021), are among the most appealing and revealing sections of the journal. 
Mentoring and supervision are not necessarily provided by the same academic, though they can be, but both roles are more Important than the research topic at any level, especially during the unsettling and uncertain times during the COVID-19 pandemic.

Topics can easily be changed, modified, redirected, and reinterpreted, among others, but the same cannot be said of talented, sensitive, encouraging, and reassuring mentors and supervisors.

After a thesis has been completed, and in several disciplines it can even be well before completion, preparation of chapters or ideas and concepts in the thesis or dissertation need to be prepared for journal submission.

Regardless of whether the supervisor/mentor is a co-author, encouragement, careful and timely reading and editing, and suggestions of possible publication outlets, are all designed to prepare a student for a possible academic or research career.

Critical and even harsh assessment of research are sometimes essential, but nothing beats a patient, understanding, encouraging, and experienced mentor and supervisor.

\section{Can Mutations in Genes Confer Antibiotic Resistance?}

The excellent and informative large team research report by Lopatkin, Bening, Manson, Stokes, Kohanski et al. (2021) investigated if clinically relevant mutations in core metabolic genes could confer antibiotic resistance.

The topic of antibiotic lethality is especially important and timely with the emergence of mutated strains and variants from the UK, South Africa, Brazil, and the USA, of the SARS-CoV2 virus that has sparked the COVID-19 pandemic. The innovative research findings that metabolic mutations arise in response to antibiotic treatment which, in turn, have implications for representative and comprehensive means in the evolution of resistance and prevalence.

Moreover, metabolic adaptation may represent resistance mechanisms that go beyond tolerance to mitigate toxic aspects of antibiotic lethality.

In future research, different mutated strains and variants, together with mild, medium, and severe, forms of COVID-19 might be investigated to broaden and strengthen the existing informative research outcomes regarding drug treatment. 


\section{Connecting Violent Entertainment and Aggression}

Regardless of personal views and entrenched opinions, any controversial connection between violent entertainment and aggression must be based on verifiable empirical facts and state of the art statistical techniques.

As discussed in O'Grady (2021), if the data seem too good to be true, such as to be raising doubts about their veracity, then that will almost certainly be the case.

There is no role in science for "alternative facts".

It is hard to believe that the first few published papers could have negotiated their way through the review process, especially based on virtually identical empirical results, which might reflect self plagiarism.

The PhD student at VCU has done the discipline, and science more generally, a great service by drawing attention to highly questionable data that have led to publications in several leading journals in psychology

Conducting a meta analysis with the questionable papers included and excluded should be sufficient to determine that they are outliers. and hence should be excluded and brought to the attention of the journals in which they were published

All data that are used in empirical research should be made available on a freely accessible website, as is the case in a number of disciplines in the social sciences and business

This is particularly important in an age when controversial opinions and confronting images are displayed regularly on social media, inciting calls for aggressive action by unrestrained commentators

Incidentally, if a leading researcher is found to be "deficient in statistical knowledge and research methods", they should be required to enroll in Statistics 101 before conducting any further research.

\section{Vaccines are Essential for Herd Immunity from COVID-19}

Herd immunity can be achieved by a sufficiently high proportion (estimated to be at last $80 \%$ ) of the population either recovering from infection, or a similarly high proportion of the population 
being protected through vaccination, in order to have an immune response to the COVID19 pandemic.

The global empirical evidence would seem to suggest that vaccination is more likely to succeed in achieving herd immunity than recovering from infection.

An obvious problem with the herd immunity mentality is that it will depend on a number of unknown factors, including the duration of the immune memory arising from safe and effective vaccines, especially against the emerging mutated strains and variants from the UK, South Africa, Brazil, the USA, and India.

As discussed in Casassus (2021), any educational campaign to encourage skeptical and distrustful members of society to be vaccinated should be supported and encouraged.

Anti-vaxxers and others who are hostile to vaccines will be difficult to persuade, but if they are among the $20 \%$ who are not vaccinated, herd immunity can still be achieved.

\section{COVID-19 Vaccines Should be Available to Everyone, Especially Healthcare Workers}

Safe, effective, durable, available, and affordable vaccines would seem to be an innate right for all individuals, all the more so for front line healthcare workers who risk their physical and mental health in caring for their patients, especially during the COVID-19 pandemic.

As discussed in Kupferschmidt (2021f), in a world where tests for COVID-19 are not free in many countries, it should not be surprising that there are many inequities in testing and vaccination.

Loss of life is tragic under any circumstances, but healthcare workers who are lost can no longer assist those who need their care the most.

The international community, led by international organizations including the WHO, need to distribute vaccines equitably to countries that are unable to afford vaccines.

If global herd immunity is to be achieved, it is estimated that at least $80 \%$ of the international community needs to be vaccinated, otherwise there will only ever be local herd immunity for the foreseeable future.

Moreover, the lower is the global circulation of COVID-19, the lower is the likelihood of emerging mutated strains and variants from the UK, South Africa, Brazil, the USA, and India, among other countries, which benefits everyone. 


\section{Which COVID-19 Vaccines Are Safe, Effective, and Durable?}

Cohen (2021b) discusses the dosing debates and the transparency issues that are affecting vaccine rollouts.

COVID-19 vaccines should be safe, effective, durable, available, and affordable, but the important question remains as to who makes such a determination, especially in the face of emerging strains and variants such as from the UK, South Africa, Brazil, the USA, and India, among others.

The important issue as to the optimal temporal duration between the prime and booster shots for two jab vaccines has not yet been determined to be 21 days (for Pfizer), 28 days (for Moderna), and between one and three months (for AstraZeneca-Oxford).

The greater is the duration between the two shots, aka the dosing interval, the greater is the likelihood of infection if the prime shot has not provided immunity.

The slew of purported "success rates" that have been reported on social media platforms, aka PR exercises, seem nothing short of "fake news".

The safety, effectiveness, duration, and dosing intervals might change over time, in which case further clinical trials would need to be conducted by reputable companies and independent drug approving agencies.

\section{Building Confidence to Face a PhD Committee, Especially During COVID-19}

Social distancing, self isolation, quarantining, and lockdowns during COVID-19 can provide a lot of thinking time, but they also make it difficult to interact with friends, colleagues, and members of $\mathrm{PhD}$ qualification committees.

Zoom, skype, and other cam-and-mic performances notwithstanding, this is where role models, such as Lady Gaga, can provide inspiration and reassurance in facing daunting $\mathrm{PhD}$ committees, regardless of how well prepared a candidate might think they are.

As discussed in the interesting and humorous working life in Dalchand (2021), making mistakes is perfectly natural, and confidence helps us to take everything in their stride, including monumental mistakes that can teach salutary lessons that are denied by success. 
I recall a PhD defence many years ago that was travelling badly, mainly because of the nervousness of the candidate, which was highlighted by incorrect answers, interspersed with "I don't know", to simple questions.

As the committee Chair, I suggested that we start again after a short break, and asked the candidate to begin with their name and academic background, to which the answer was a kneejerk "I don't know".

These things can and do happen when nerves take hold, as any experienced academic knows.

We all live and learn about self confidence and dealing with self doubt, especially from inspirational role models.

\section{Academic Openness and National Security During COVID-19}

As discussed in Lodish, Chen and Sharp (2021), attracting outstanding foreign scientists and students is frequently helpful to both the source and recipient countries, but a balance between academic openness and national security is essential at all times, including during COVID-19.

There are benefits to both the domestic recipient and foreign source countries, but intellectual property must be protected against those who might seek to benefit from innovative discoveries without respecting the rightful owner(s) and ownership of R\&D, patents, copyrights, and trade secrets, among others.

Even when espionage is not suspected, when international academics are required to sign off on intellectual property while working in China, as an example, this is tantamount to claiming ownership of intellectual property without covering the actual costs of the discoveries by compensating the creators appropriately.

It is one thing to demand full disclosure in examining the activities of academics who might be suspected of scientific and financial misconduct through illegal and undeclared contacts with foreign governments, but it is an altogether different proposition in failing to respect and serve due process and natural justice.

100. Antiviral Activity Against SARS-CoV-2 
The expert medical scientists Wong and Damania (2021) report their intriguing and informative perspectives on the dependence of SARS-CoV-2 on a range of host pathways, and how the potency of presently approved vaccines for the original wild-type coronavirus (COVID19) might be hampered by the emerging strains and variants in the UK, South Africa, Brazil, the USA (especially California), and throughout India, on global morbidity and mortality.

Any drug, including remdesivir, that is found to possess antiviral activity against viral infections, specifically SARS-CoV-2 during the COVID-19 pandemic, would seem to be worth investigating in clinical trials, especially on humans, while ensuring that toxicities are prevented.

Antivirals are typically found to be most effective against mild and medium COVID-19, whereas some vaccines have been found to be especially effective against severe cases of the disease.

The reduced effectiveness and protection of the approved vaccines against the emerging escaped mutants is essential to investigate, especially when the mutated strains and variants are expanding at a much faster rate than is the original coronavirus, and display greater resistance against the vaccines.

\section{Ignoring the Status of Retracted COVID-19 Papers is Academic Fake News}

In just over one year, the number of published papers on COVID-19 would run into the millions, if the more than one million papers listed on the "WHO COVID-19 Global literature on coronavirus disease" is any guide.

As discussed in Piller (2021), a unknown proportion of the published papers are likely to have been rushed through the editorial process, with associated problematic issues clouding a failure to examine their scientific accuracy.

Yet even retracted and, by definition, disgraced papers in leading journals continue to be cited in ignorance, including in leading journals, if the failure to cite their status is any guide.

This disappointing failure is, in essence, a case of academic fake news, which would seem to suggest that academics are not immune from the willful or negligent aziness that seems to afflict a high proportion of the misguided posts on many social media platforms.

Such laziness is inexcusable as there are leading retraction database websites that are easily accessed. 
In cases where retracted papers are based on clinical laboratory trials, this could lead to risking the lives of patients and compromising public healthcare, which make scientific fraud look relatively tame in comparison.

\section{Vaccinating Children and Adolescents Against COVID-19}

As discussed in Couzin-Frankel (2021), with the COVID-19 pandemic still expanding around the globe, with approved vaccines facing the emergence of more highly transmissible and infectious mutated strains and variants from a number of sources, including the UK, South Africa, Brazil, USA, and India, vaccine trials are expanding to include children and adolescents, for whom no existing vaccines have yet been approved in terms of their safety and efficacy

Even if the young, as a group with ages from 3 to 17, are less likely to be infected, they are still able to transmit COVID-19 to others, and can also suffer the effects of long haul COVID, so that vaccinating them is crucial to reducing infection rates.

If the young are to be vaccinated in order to return to a semblance of normality without relying on masking, there must be extensive clinical trials to provide clear outcomes that they are safe, effective, and durable for children and adolescents.

\section{Inhibiting SARS-CoV-2 by Enhancing Host Cell Infection}

Although published a few months ago, and before the expanding second and third waves of COVID-19 infection, the emerging highly transmissible and infectious strains and variants from several sources, including the UK, South Africa, Brazil, USA, and India, and the distribution of several approved vaccines against the original wild-type coronavirus disease (COVID-19), the informative perspective on inhibiting SARS-CoV-2 by enhancing host cell infection remains important and topical.

The empirical finding discussed in Kielian (2020) that might be able to explain a loss of sense of smell in long haul COVID-19 patients would be made stronger if the effects of taste could be accommodated. 
The impact of enhancing host cell infection on the safety, efficacy, and duration of presently approved vaccines, especially against the escaped mutants, would make the promising outcomes even more important from a practical perspective.

\section{Testing COVID-19 Megatrial Therapies and Treatments}

Whether it is an international or WHO megatrial, it is essential to test experimental therapies and treatments for COVID-19 to determine which vaccines are safe, effective, and durable against the original wild-type coronavirus (COVID-19) and the emerging strains and variants from the UK, South Africa, Brazil, USA, India, and Russia.

As presented in an informative discussion in Kupferschmidt (2021g), the only global megatrial that was organized under the WHO's Solidarity trial seems to have ground to a halt.

It is undoubtedly difficult to organize an ongoing international trial that involves a large number of researchers and institutions, and a far larger number of patients, but if the WHO cannot conduct and coordinate an international megatrial on testing, selecting, and prioritizing treatments and vaccines, then who can?

Extensive, randomized, and controlled clinical trials undoubtedly provide the most reliable cost-benefit risk analysis of medical research outcomes in terms of infections and deaths, the absence of which is likely to lead to inconclusive outcomes that are based on small numbers of patients.

Testing a wide range of experimental therapies and treatments in a global effort is essential to mitigate the COVID-19 pandemic.

\section{New Scientific Frontiers in the Time of COVID-19}

It is reassuring to read the prescient editorial by Mertz and Mishra (2021) regarding a new frontier for Science, a leading outlet for novel scientific research, as well as a new frontier for science, in general, during the time of COVID-19.

It is unfortunate and disappointing that the fake news being disseminated widely on news and social media platforms downplays the role of science in dealing with COVID-19, despite fake 
news having started before the previous administration took office, and well before the pandemic started.

Given the presence of climate change deniers at the highest levels in society, including national governments, it is hardly surprising that anti-vaxxers and anti-maskers have established a loud voice across age, gender, demographic, and socioeconomic cohorts.

All leading research institutions, academic journals, and scientists need to make their voices heard loudly, clearly, and repeatedly in trying to educate society about what is based on science, and what is not.

Education is a foundation of any civilized society and, even though it will take time, failure to provide scientific information and evidence-based communication has consequences that will last forever.

\section{Questionable Data Throw School Openings During COVID-19 Under a Spotlight}

As discussed in Vogel (2021), opening schools in Sweden during the early months of a pandemic might be regarded as a courageous decision, especially if the safety of school children cannot be guaranteed through a lack of information regarding the precautions that should be taken.

Children have not been tested in clinical trials of COVID-19 vaccines, so the safety, efficacy,

and durability of vaccines as applied to children are not yet known, though it is known that asymptomatic children can transmit the disease to adults, especially the aged.

The herd immunity strategy of protecting the most vulnerable in society via vaccination while establishing protection through infection for the rest of the population relies on recovery of those who have not been vaccinated.

Staff at schools have been infected, while the emerging mutated strains and variants from the UK, South Africa, Brazil, USA, India, and Russia will likely increase transmission and infection, as well as recovery.

Protecting all cohorts in society is essential during a pandemic, and questionable data throw school openings during COVID-19 under a spotlight.

107. Supply Chains and COVID-19 Vaccines: The Need for Speed 
There is no questioning the need for speed in producing and distributing COVID-19 vaccines from medical, economic, financial, and social perspectives.

The detailed and informative research by a team of experts in Castillo, Ahuja, Athey, Baker, Budish, Chipty, Glennerster et al. (2021) makes it clear that improving market design is essential for accelerating the global supply of safe, effective, and temporally durable vaccines.

Reducing the number of shots, as well as the duration between the prime and booster shots for a two-shot regime, to a single shot would contribute to improving the supply chain, as would lowering the dose of any shot.

If global herd immunity is to be achieved, a high p;;proportion of the global population will need to be vaccinated, as the infection strategy has a number of associated risks, including a failure to reach a sufficiently high percentage (usually estimated to be at least $80 \%$ ) of the global population that recovers from infection.

The mantra from the film "Top Gun" was "I feel the need ... the need for speed!", which can be facilitated by international organizations, such as the WHO, becoming involved in the global supply chain strategy.

The consequences in not accelerating the need for speed in supplying and distributing COVID19 vaccines will be an unwanted continuation of the pandemic, with dire consequences for the global community.

\section{Research Lessons from COVID-19}

As discussed in the informative report by Collins (2021), the COVID-19 pandemic has had medical, public healthcare access, healthcare and hospital costs and insurance, economic, financial, and social impacts, the informative editorial from a health expert on how we must not become complacent in the research lessons from COVID-19 is a call to arms for all researchers across a wide range of disciplines.

The warp speed in which vaccines have been developed and distributed globally has been a milestone in research intensity, clinical trials, testing regimes, and supply chain management, with further clinical trials being conducted regarding the performance of approved vaccines against the mutated strains and variants that are emerging globally. 
Including those members of society who have been excluded from existing clinical trials, such as the young, the aged, racial and ethnic minorities, those at the lower end of the socioeconomic distribution, and incarcerated individuals in prisons and jails, should be included in testing samples in order to represent the population more accurately.

The temporal durability of single and two-shot vaccines needs to be addressed to provide greater reassurance to those who might presently be wary of which vaccine might be most suitable, bearing in mind their known side effects.

Complacency is not an option.

\section{Transmissibility and Impact of the UK COVID-19 Lineage}

As discussed in Davies, Abbott, Barnard, Jarvis, Kucharski et al. (2021), the emergence of several mutated strains, variants, and lineages of the original wild-type coronavirus (COVID-19) in the UK, South Africa, Brazil, USA, India, and Russia has raised concern regarding the safety, efficacy, and unknown durability of the approved one- and two-shot vaccines, as well as their observed side effects.

Using various statistical and dynamic modelling approaches, an impressive team of medical researchers investigated and estimated the transmissibility and impact of the UK COVID-19 lineage, which was found to have a higher reproduction number than other variants.

For public healthcare policy purposes, it is essential to evaluate the characteristics of the UK lineage as it has begun to dominate the original and subsequent lineages globally, with its estimated growth rate remaining among the highest as a function of lineage age.

Based on a range of assumptions underlying multinomial splines and logistic regression analyses, the reproduction number was estimated, although no diagnostic checks seem to have been presented to determine the robustness of the empirical results.

Using tests on previously estimated reproduction numbers arising from different models, as well as conditioning factors, can lead to pre-test bias, which undermines the statistical significance of the empirical analyses.

Each model was estimated using time series data for the period 1 March - 24 December 2020, which was at the beginning of the global distribution of vaccines, so extending the data set by even 
two months would help in a greater understanding of the effects of vaccination on the transmissibility and impact of the UK COVID-19 lineage.

\section{Neutralizing the Impact of the UK COVID-19 Lineage}

The informative and beneficial research report is presented by a team of medical experts in Muik, Wallisch, Sänger, Swanson, Mühl, Chen, Cai et al. (2021) on neutralizing the impact of the UK COVID-19 lineage using vaccine-elicited human sera that were obtained 3 weeks after the booster shot of a two-shot vaccine.

The observed decrease in neutralization was clearer for participants aged under 55 years, and the vaccine was found to provide significant protection against the UK variant in a comparison with the original wild-type coronavirus (COVID-19).

Although the mutated UK lineage seems to have dominated global COVID-19 infection, it would also be useful for public healthcare policy if the vaccines were tested against the other mutated lineages that have emerged in South Africa, Brazil, USA, India, and Russia.

Updating the data beyond 14 November 2020 would accommodate the production and distribution of a range of vaccines, including single-shot vaccines, and the emergence of escaped mutants, such that using vaccine-elicited human sera 3-4 and 12 weeks after the single shot, could strengthen the impressive and promising findings of the research outcomes.

Maintaining the safety and efficacy of any vaccines arising from these observed outcomes is essential, as would any clarification regarding the as yet unknown temporal durability of protection arising from the vaccines.

\section{Within-host Diversity and Transmission of COVID-19}

As presented in Lythgoe, Hall, Ferretti, de Cesare, MacIntyre-Cockett et al. (2021), the ability to monitor the transmission of COVID-19, and to predict and identify new strains, variants, and lineages of the original wild-type coronavirus (COVID-19) that have emerged in several countries, including the virulent UK lineage, are among the most important issues facing the global community in terms of medical research, healthcare public policy, economic, financial, and social issues. 
The important research results reported by a team of medical experts concerns the viral withinhost diversity at the population level and transmission of the UK variant.

The main research findings are that escaped mutants are likely to arise infrequently, but could spread rapidly if they are highly transmissible, infectious, and contagious, as seems to be the case of the UK variant.

The unknown effects of combinations of known and unknown variants, as well as the safety, efficacy, and temporal durability of combinations of one- and two-shot vaccines, awaits the outcomes of clinical trials.

As escaped mutants may prevent herd immunity through natural infection and recovery, or through extensive vaccination programs, complacency must be avoided at all costs.

Estimation of thresholds, including within-host diversity, is fraught with difficulties in terms of the assumptions underlying the estimated linear (without threshold) and nonlinear (with threshold) models, as well as the estimation and statistical testing methods that are used.

The apparent absence of diagnostic checks makes it difficult to determine the robustness of the otherwise informative and impressive empirical results.

\section{Partial Immunity from COVID-19 Vaccine Dosing Regimes}

With supply chain blockages in the process of producing and distributing vaccines against COVID-19, the impressive team of medical researchers in Saad-Roy, Morris, Metcalf, Mina, Baker et al. (2021) report on the epidemiological and evolutionary considerations of SARS-CoV2 vaccine dosing regimes.

The safety, efficacy, and presently unknown temporal strength and durability of the prime shot of a two-shot vaccine, or a single dose of a one-shot vaccine, lead to questions about the robustness of protection from infection by COVID-19.

An impressive array of epidemiological and evolutionary simulation models based on one- and two-dose vaccination regimes is used to evaluate the robustness of COVID-19 vaccine dosing regimes.

The sophisticated model and underlying assumptions governing the transitions between the infection and immunity classes are presented as deterministic rather than stochastic equations, which can affect the numeral simulation results. 
The analysis of seasonal variations may be problematic as there are insufficient data to determine if there is seasonality in infection rates from COVID-19.

Although a range of statistical tests is presented, the apparent lack of any diagnostic checks makes it difficult to determine the robustness of the empirical results in seeking to determine the robustness of the vaccines.

\section{Great Challenges Need Great Ideas, Especially for COVID-19}

Computer software programs based on artificial intelligence (AI) are discussed in a detailed and informative report by Service (2021a) on an efficient and hyper speed way of investigating the connections across a large number of variables, especially drug design against viruses such as SARS-CoV-2 that causes COVID-19.

This can be very helpful when numerous drug compounds and target inhibitors are being investigated and laboratory tested for possible synthesizing of compounds that block human enzymes against infection of cells.

Improving the potency of the currently approved COVID-19 vaccines, especially against the virulent emerging strains, variants, and lineages from the UK, South Africa, Brazil, USA, India, and Russia, is also possible through the use of AI and supercomputers.

AI can also assist in investigating repurposed compounds that can affect the body's reaction to infection from a virus, or the virus itself.

As COVID-19 and its various escaped mutants continue to cause drastic and dramatic global impacts on health and medical facilities and treatments, the economy, and society, any assistance that can be provided, especially through scientific approaches to advanced computer software programs and artificial intelligence against COVID-19 and any other emerging viral threat, would be welcomed with open arms by a long suffering and fatigued world.

\section{Hoarding COVID-19 Vaccines is Unethical}

The informative and prescriptive report by two expert commentators in Cohen and Kupferschmidt (2021) is concerned with what to do with COVID-19 vaccine surpluses, namely to hoard or to donate to those who need it. 
Many high income countries prepurchased several different vaccines when little was known about their safety, efficacy, and temporal duration, although may single- or two-dose vaccines have been shown through testing in clinical trials to be safe and effective, although little is yet known about the durable memory of the vaccines, and how they might be extended through booster shots.

Although 11 countries are included in the table, it should be emphasized that the EU has supported Italy in blocking the shipment of 250,000 AstraZeneca vaccines that had been ordered.

Moreover, Australia has made a formal request to AstraZeneca and the EU authorities to access one million contracted supplies of AstraZeneca vaccines so that they can be shipped from Europe to Papua New Guinea, where the pandemic is out of control, primarily through a lack of testing.

For herd immunity to take effect through a high percentage of the global population, estimated to be at least $80 \%$, to be protected through vaccines, the supply chain distribution channels must be directed to every country, regardless of their ability to pay for the vaccines.

Hamlet's soliloquy might be paraphrased as: "To hoard or not to hoard", with the response that hoarding is unethical when lives are at stake.

\section{COVID-19 Vaccination Passports for International Travel}

The informative and prescriptive editorial by Dye and Mills (2021) is essential reading for the aviation industry, travellers, and travel-related companies, including hospitality and tourism.

A number of international organizations, including the WHO, already provide certificates for some vaccines and diseases, and this should be extended to include COVID-19.

It would be useful, convenient, and safe for vaccination passports to be linked directly to passports for security purposes, and should state where, when, and the type of vaccine that was administered.

If a passport is due to expire within 6 months, the vaccination certificate could be transferred to the new passport.

Exclusions and exemptions should be considered carefully, especially as vaccines are intended to protect transmission and infection.

Vaccination passports need to be based on science, with equity, global or travel bubble acceptance, and access available to all. 


\section{Naming COVID-19 Strains, Variants, and Lineages}

It is not clear whether the authors, Karim, de Oliveira and Loots (2021), of this one-sided report expect the news media and individuals globally refer to any virus by the title VOC-202012/01 (B.1.1.7, 501Y.V1, 20I), rather than the UK mutation.

The general classification of COVID-19, and the mutated strains, variants, and lineages that have emerged in the UK, South Africa, Brazil, USA, India, and Russia, are widely referred to by the country of first observation.

Such a naming designation is a fact of life, based on the Keep It Simple (KIS) principle.

Regarding the so-called "China virus", who uses the term other than former President Donald Trump?

As for recommendations arising from the $\mathrm{WHO}$, the latest information about their investigation in Wuhan, China, is that it is biased, distorted, and was heavily influenced by China.

The authors do not mention the so-called "Spanish flu", but should have done so if they believe that no country designation should be attributed to any virus or disease.

\section{Zoom Meetings for Mothers During COVID-19}

Working life and career stories are deeply personal perspectives on how academics have come to accept with their responsibilities in a COVID-19 world.

As discussed in Dhen (2021), working from home while caring for young children, especially if they insist on being loud and uncontrollable during zoom meetings, with cam and mic, can be a demanding handful.

A situation can deteriorate quickly during large meetings, but I frequently have one-on-one zoom calls with colleagues who have young children, who are capable of emphasizing what is really important in life.

In addition to partners, professional colleagues can provide emotional support during zoom meetings by reducing stress and anxiety for working parents, especially working mothers who bear the weight of responsibility and caring for young children. 
Anyone who regards academics with families as displaying a lack of commitment or engagement to their professional work needs to think carefully if their own priorities are in order.

With respect, it is simply polite to inquire if a parent, regardless of gender, might need a moment or two to deal with any possible difficulties arising from crying children, especially if it is interfering with an academic or administrative zoom discussion.

\section{Research is Supposed to be Enjoyable, COVID-19 or No COVID-19}

Good research is not easy, otherwise everyone would be doing it.

Although this does not necessarily hold for all graduate research students and academics, as in the interesting discussion in Wright (2021), research is supposed to be challenging, satisfying, rewarding, and enjoyable, COVID-19 or no COVID-19.

Publishing papers in leading academic journals, to be followed by citations in journals of equal quality, can be shared with supervisors, colleagues, fellow graduate students, and family members through email messages and zoom meetings.

A graduate degree is not suitable for everyone as a career path, and not everyone is suited for a graduate degree.

Depending on the discipline, some academics and graduate research students wish to work alone, while others prefer to work in collaborative research teams.

It is a case of horses for courses.

\section{Asymptomatic and Presymptomatic COVID-19 Cases}

The unknown, though potentially lengthy, incubation period of SARS-CoV-2 makes it difficult to detect, especially among asymptomatic and presymptomatic persons who do not display obvious illness and are unaware that they have been infected, and hence are unlikely to be tested.

As reported in the challenging perspective by Rasmussen and Popescu (2021), where symptoms of COVID-19 are not evident, the lack of testing makes mitigation of the disease difficult, especially as exposures are typically unknown, which can understandably make accurate contact tracing difficult. 
The emerging mutated strains, variants and lineages, in countries such as the UK, South Africa, Brazil, USA, India, and Russia, that are more highly transmissible, infectious, and contagious, makes asymptomatic and presymptomatic cases of even greater concern.

Data on symptomless persons are not available as they are unknown and inherently complex, but the absence of asymptomatic and presymptomatic cases in the reported data would lead to a downward sample selection bias in the number of detected cases.

Community surveillance screening has occurred in targeted aged care facilities, nursing homes, and professional sports and entertainment industries but, as they do not reflect the broader community, the data that are available are not representative of the broader asymptomatic and presymptomatic cases.

To be more specific, facilities and industries other than those mentioned above do not have the resources that are needed for community surveillance screening and testing.

Moreover, the numbers of asymptomatic and presymptomatic cases in children, adolescents, and younger adults, who are unlikely to be in aged care facilities, nursing homes, or in the professional sports and entertainment industries, would be unlikely to be involved in community surveillance screening, thereby biasing the number of reported cases downward even further.

The secondary transmission and infection effects from those who are asymptomatic and presymptomatic are also unknown, which makes mitigation and control of the spread of COVID19 infection even more challenging as such individuals are likely to be unknowingly transmitting the virus and disease in the community.

Vaccinating as many members of the global community would reduce transmission as they would incorporate asymptomatic and presymptomatic cases, but this is not likely to occur in the near future.

\section{Impaired Immunity Through Evolving SARS-CoV-2 Variants}

As discussed in McCormick, Jacobs and Mellors (2021), the evolution of the SARS-CoV-2 virus through mutation and natural selection can impair the effectiveness of vaccines for COVID19, and lead to more highly transmissible, infectious, and contagious disease. 
Combinations of the original wild-type coronavirus (COVID-19) with some or all of the emerging strains, variants, and lineages that have emerged globally could lead to faster and more virulent variants that may impair immunity against the disease.

Viral diversification may also impair immune responses against acute infection throughout the respiratory tract, for which evidence is needed from additional clinical trials and empirical outcomes via global distribution of vaccines

It is worth noting that, of the leading six countries with confirmed total cases, namely, USA (specifically, California and New York), Brazil, India (numerous variants), Russia, France and UK, only France does not have a variant that started in the host country.

Nigeria and Uganda might also have hosted their respective variants, but the relatively low number of confirmed cases, and especially deaths, is almost certainly a result of inadequate reporting of official statistics.

The observed propensity of intra-host evolution has shown that SARS-CoV-2 can evolve multiple distinct lineages within the same individual, which is a worrying development if it were to hold across a large segment of the population.

\section{How Many Mentors is Optimal, Especially During COVID-19?}

The career stories in the working life section, such as in Moore (2021), are deeply personal and make for enjoyable reading.

Whether more mentors lead to better scholarship, research, publications, and citations, will likely vary by discipline, topic, the graduate research candidate, and the interactions among the mentors.

The choice of mentors depends not just what each brings to the table, but also how their synergies interact to assist the mentee, especially during COVID-19.

Depending on the field, especially where collaborative team research is involved, each member of the team brings a specific set of skills, whereas in many areas in the social sciences, humanities, and business, graduate research students are expected to have some idea of what they might wish to investigate in undertaking independent research.

A mentoring network is an excellent strategy when possibly conflicting advice is sought from a variety of experts across cognate fields of research. 
The four helpful and informative suggestions make it clear that the writer has gained a lot of knowledge about what mentors are able to provide, which is a large part of the graduate learning experience, much of which involves the KIS (Keep It Simple) principle.

\section{Why is Trust in COVID-19 Vaccines Volatile?}

The challenging editorial in Larson and Broniatowski (2021) is almost as confronting as the apparent volatility in societal trust in any COVID-19 vaccines, which is based heavily on misinformation, conspiracy theories, and fake news that are disseminated through social media platforms.

Why the safety of AstraZeneca should be questioned is difficult to answer, especially as there is no scientific evidence based on clinical trials that it might not be safe, and doubts are being cast in countries that are struggling with the pandemic.

Moreover, the widely-used vaccine has passed the public trust test in the UK, and mitigates against vaccine hesitancy.

Trust can be difficult to establish, but easy to dismantle, so clear, simple, calm, and repeated scientific reporting through public health channels is essential.

It is important to distinguish between anti-vaxxers, who are misguidedly ignorant, and the vaccine-hesitant, who are confused, and lack confidence and trust in the mixed messages that are being widely distributed.

Convincing those who believe that vaccines are dangerous to health, while COVID-19 is not, is a difficult task, but one that needs to be confronted if society is to overcome the pandemic.

\section{Practical Carbon Capture in a COVID-19 World}

The COVID-19 pandemic has had disastrous effects on the economy, financial institutions, and global public health, but it has also had some unintended benefits, including substantially reduced air travel, and reductions in private transportation from working at home.

As discussed in Service (2021b), given that renewable and sustainable energy sources are not yet capable of replacing fossil fuel energy sources, capturing carbon dioxide, $\mathrm{CO} 2$, using new 
generation chemical traps that are capable of reducing costs of capture by not boiling and then recondensing water, are a welcome addition to existing approaches.

Existing chemical technology is expensive because they require substantial energy use in dissolving chemicals in reusable water, boiling water to release the $\mathrm{CO} 2$, and re-condensing the water vapor.

However, new technology might be able to reduce the costs of carbon capture by an estimated $50 \%$ within the next decade or two, to levels that will be necessary to make carbon capture cost effective and economically viable, by using liquid organic solvents and avoiding the use of water.

In addition to new chemical technology for capturing carbon dioxide, it is worth considering environmentally friendly technology, such as in the use of forests where, among a range of trees that are capable of attracting and storing carbon dioxide, eucalypts are among the most efficient with high absorption rates.

It follows that the logging of forests, as well as uncontrolled forest bush fires, such as those in the last year in Australia and the USA, the carbon dioxide that had been stored will be released into the atmosphere.

\section{Redesigning Vaccines to be Effective Against COVID-19 Variants}

Further to the report by Luchsinger and Hillyer (2021), the evidence arising from any countries with the mutated variants of COVID-19 that have emerged from the UK, South Africa, and Brazil seems to show that, while they remain safe, they are more highly transmissible, infectious, contagious, and deadly than the original wild-type coronavirus (COVID-19) that was detected in Wuhan, China in late 2019.

The escaped mutant from Brazil has been infecting those aged 18-45, with up to three times the deadly consequences.

Moreover, the new variants seem to be acting like new coronaviruses, leading to the effectiveness of existing vaccines being reduced in potency for acquired immunity.

Reinfection from mutated strains, variants, and lineages, as well as long haul COVID-19 effects, will need to be investigated carefully using dynamic longitudinal panel data samples to evaluate how existing vaccines might need to be redesigned to render protection against the mutations. 


\section{The Science Behind the AstraZeneca COVID-19 Vaccine}

As discussed in Vogel and Kupferschmidt (2021a), there have been substantial news and social media platforms about concerns and loss of confidence in the AstraZeneca COVID-19 vaccine, but where is the science based on clinical trials?

This is of concern predominantly in Europe, where AstraZeneca was intended to be the key vaccine in mitigating the pandemic, as well as in many countries where the relatively low cost of the vaccine, and simple storage requirements, are appealing, as exemplified by the WHO's intention to use the vaccine in low-income countries.

After numerous European countries temporarily suspended the use of the vaccine, the lack of any science underlying the purportedly unusual blood clot side effects, low platelet counts, and rare hematological disorders, primarily in those under 55 years of age, led the European Medicines Agency (EMA) to approve the vaccine as safe.

Alleged misinformation about the efficacy of the vaccine is a separate issue, and the company has tried belatedly to respond to the complaints about cherry picking "outdated" information from clinical trials in support of efficacy.

The loss of confidence in any vaccine is of concern to governments and healthcare officials, especially during a pandemic, and also where supply chain distributional issues are problematic.

Leaders in science, especially medical researchers, should emphasize and highlight the benefits of vaccinations if they are shown to be safe and effective, even if the temporal duration of any COVID-19 vaccines is presently unknown.

\section{Is it Sensible to Restrict AstraZeneca by Age?}

All vaccines have side effects, some more so than others, especially when clinical laboratory trials and development of vaccines for COVID-19 have progressed at warp speed.

As discussed in Vogel and Kupferschmidt (2021b), a small number of AstraZeneca vaccine recipients have been found to experience rare blood coagulation, especially for women under 65 years, and low platelet counts, especially for individuals recently injected with a specific batch, so much so that its safety has been called into question. 
A number of countries have restricted the vaccine to those above a certain age, be it 50, 55, or 60 years of age, as younger cohorts seem to have a greater risk of blood clotting.

The added presumption that younger adults are less likely to be infected by COVID-19 has been belied by the findings that the mutated strain, variant, or lineage from Brazil has targeted those aged from 18 to 45 years, with more highly transmissible, infectious, contagious, and deadly effects than the original wild-type coronavirus (COVID-19).

Several national risk assessment panels and medical societies have approved the safety of the vaccine, especially in comparison with the damaging consequences of not being vaccinated against COVID-19.

Unlike some European countries, the UK has not reported an unexpected number of vaccinated patients suffering from blood clots, although evidence has not been presented according to age.

Convincing individuals that vaccines are safe and effective, albeit with unknown temporal duration, is difficult enough without the added concerns and confusion regarding unusual and rare side effects about protection against COVID-19.

\section{Protecting Scientists from COVID-19 Burnout}

Life can present difficult hurdles and challenges, especially during a pandemic.

As discussed in Wadman (2021b), as the extent and serious consequences of COVID-19 continue to mount, all members of society are facing long periods of mental stress, none more so than frontline healthcare workers and scientists and medical researchers who are at the forefront of investigating vaccines to protect against the virus and disease.

COVID-19 fatigue seems to be affecting every segment of society, including those who are faced with the challenging task of dealing with sick patients and developing vaccines against the original wild-type coronavirus (COVID-19) and the more highly transmissible, infectious, contagious, and deadly mutated strains, variants and lineages that have emerged globally.

The hyper speed at which vaccines have been developed and approved for distribution has placed extreme stress and fatigue on the scientists who have been involved in the research and testing of the vaccines.

Trying to convince the vaccine hesitant with clear and consistent messages about the safety and efficacy of the approved vaccines, especially when conflicting messages are distributed widely 
in news and social media platforms, also places stress and anxiety on those who are tasked with the responsibility of educating the concerned public.

The pandemic has raised challenges that can be exciting and fulfilling to those investigating COVID-19, but has also constrained the quality of well-being through mental health pressures that are not likely to be alleviated in the near future.

Society and governments need to step in to reduce the burnout toll by lightening the workload on scientists and medical researchers, as well as frontline healthcare workers, who are faced with an increasingly heavy workload burden as the number of cases increases in expanding higher waves globally.

\section{Adding Technology to the NSF for Scientific Competitiveness}

The discussion in Conn (2021) is concerned with expanding funding for the NSF, which might be changed to NSTF to include Technology, is to be lauded as a direct infusion of funds will improve scientific competitiveness both domestically and internationally.

The addition of Technology that is needs-directed or use-inspired, to Science, aka basic

research, is an innovative development that will be strongly supported by the scientific community and associated organizations, as the funding and recognition by the Federal Government is long overdue.

Three targeted and timely areas include the high-tech focus areas of artificial intelligence, quantum information sciences, and advanced materials, each of which is being developed rapidly at a global level.

In addition to providing research funding and financial support across all levels of tertiary academic institutions, the extended program will support the protection of intellectual property and $R \& D$ through industrial commercialization of patents and trademarks.

Politicization of the new Foundation would impede scientific progress, research funding, and protecting intellectual property, and should be resisted at all costs.

Establishing equity and expanding diversity in the disbursement of funding for Science and Technology should be a primary goal of the expanded program.

\section{Causality Between COVID-19 Cases and Vaccination}


As of 9 April 2021, India had the third highest number of confirmed COVID-19 cases, behind the USA and Brazil, and the fourth highest number of deaths behind the USA, Brazil, and Mexico (https://www.worldometers.info/coronavirus/).

Following the discussion in Chandrashekhar (2021), any perception that the pandemic was under control in India was shown to be an illusion with the onset of yet another new wave of cases and deaths, with a concentration in eight states, including Maharashtra, which has India's commercial centre of Mumbai as the capital.

With two approved vaccines, it might be perceived that vaccination might be widespread, but problematic supply chains issues have prevented the broad distribution across the nation.

The direction of causality is generally regarded as being from vaccinations to total confirmed cases but, in the absence of widespread vaccinations, bivariate causality might be from total confirmed cases to vaccinations.

Herd immunity might arise from a sufficiently high percentage of the population having recovered from infection, but the overall threshold at approximately $22 \%$ is nowhere near the estimated $80 \%$ that is required for the strategy to work.

For this reason, a high percentage of the population needs to be vaccinated to achieve herd immunity, if societal healthcare requirements such as personal hygiene, masking, social distancing, and contact tracing continue to be observed.

The safety and efficacy of approved vaccines has not yet been established, especially against the numerous mutated domestic strains, variants, and lineages of the original wild-type coronavirus (COVID-19), as well as of the more highly transmissible, infectious, contagious, and deadly mutations that have emerged from the UK, South Africa, and Brazil.

The latest surge in infections must be mitigated through educating the vaccine hesitant that public healthcare protection of the nation depends on a massive increase in vaccinations.

An update as of 15 April 2021 shows that India had overtaken Brazil to have the second highest number of total confirmed cases of COVID-19, behind the USA, and still had the fourth highest number of deaths associated with the pandemic, after the USA, Brazil, and Mexico.

One day earlier, the number of cases was the highest recorded in a single day of any country since the beginning of the pandemic. 


\section{Expanding the Narrative Behind the Development of COVID-19 Vaccines}

There would be few medical specialists better placed anywhere to present an informative editorial on the historical development of any vaccines, including for COVID-19, than Fauci (2021), the Director of the National Institute of Allergy and Infectious Diseases, NIH.

The development of a variety of safe and effective vaccines at warp speed is unprecedented, being based on RNA platforms and adapted immunogens, but it might also be responsible for vaccine hesitation in the population, even among the highly educated.

Previous research on SARS (aka SARS-CoV-1) and MERS have enabled the development of vaccines at Moderna, Pfizer, and BioNTech.

There are many known unknowns, including the temporal durability of any vaccines, and their efficacy against the mutated strains, variants, and lineages of the original wild-type coronavirus (COVID-19), but these are currently under clinical investigation, probably at warp speed.

\section{Confirmed Cases, Deaths, and Vaccinations Against COVID-19 in Brazil}

As of 15 September 2021, Brazil had the third highest number of confirmed COVID-19 cases, behind the USA and India, and the second highest number of deaths behind the USA (https://www.worldometers.info/coronavirus/).

Manaus is the seventh largest city in Brazil, at over 2 million and, as the resurgence of COVID19 , as well as the mutated lineage that has emerged in the country, has debilitating ramifications for everyone, it is of importance to examine how the original wild-type coronavirus (COVID-19) and the escaped mutant are behaving.

The impressive research report by Faria, Mellan, Whittaker, Claro, da S. Candido, Mishra et al. (2021), analyzed genome sequencing of viruses sampled in Manaus between November 2020 and January 2021, which revealed the emergence and circulation of a novel mutation.

Manaus is a critical city to examine as it is the capital of the worst affected state in the country, namely Amazonas, in the middle of the Amazon Rainforest at the confluence of the Rio Negro River and Solimões River, with both tributaries forming the Amazon River.

The report found that the Brazil lineage was more highly transmissible than the original strain, and that infection with COVID-19 reduced protection through immunity. 
The second wave of infections and deaths in Manaus showed that the attack rate, which is an estimate of the final size of an unmitigated pandemic, after correcting for cases without a detectable antibody response and antibody waning, showed that the level of previous infection was not sufficient to prevent a rapid resurgence of the pandemic in late 2020 and early 2021.

Clinical trials are needed to determine the safety and efficacy of currently approved vaccines by Pfizer, Moderna, AstraZeneca, and Johnson \& Johnson, among others, against the Brazil lineage.

\section{Concluding Remarks}

The highly infectious and rapidly mutating SARS-CoV-2 virus that causes the COVID-19 disease is confronting the international community with few palatable options. The research output on COVID-19 has been revolutionary, especially in the medical and biomedical sciences, where the search for a vaccine is essential for the world to have a semblance of normality in a COVID19 normal world. Much of the advanced research has been distributed in the leading medical journals, where the latest medical research is distributed on a daily basis.

The Science journals also have a new section on "Coronavirus: Research, Commentary, and News" that strives "to provide the best and most-timely research, analysis, and news coverage of COVID-19 and the coronavirus that causes it. All content is free to access."

The purpose of this paper was to provide a supplement the existing reviews and perspectives, and a critical assessment of 144 interesting, informative, and highly topical research papers that cover several highly topical and diverse medical issues in the COVID-19 era that have been published on general medicine, translational medicine, immunology, and robotics in Science.

The diverse topics include the effectiveness of vaccines against mutations, establishing trust is hard work, distrust is easy, transmission of COVID-19 between mink and humans, the war against COVID-19 deniers, SARS-CoV-2 is already endemic, COVID-29 efficacy, safety is essential for any vaccine for SARS-CoV-2, aerosol transmission of SARS-CoV-2, pre-existing immunity to COVID-19, de novo mutations, and frequency and duration of COVID-19 reinfection, SARSCoV-2 transmission between mink and humans, further known unknowns about $\mathrm{T}$ cell immunity to COVID-19, modeling confusion to clarity in a COVID-19 world, determining serological correlates of COVID-19 severity, what came first, effective COVID-19 vaccines or trial ethics?, 
safety et al. requirements for COVID-19 vaccines, controlling transmission heterogeneities and controllability of SARS-CoV-2 and COVID-19, transmission dynamics for a seemingly never ending COVID-19 pandemic, susceptibility to and long hauler effects from severe COVID-19, attack rates of COVID-19 in the Brazilian Amazon, humoral immunity to severe COVID-19, phylogenetic analysis of COVID-19 superspreading events, seasonal flu versus seasonal COVID19, controlling the spread of COVID-19, antibody responses to severe COVID-19, direct and indirect COVID-19 effects on the heart, government interventions against COVID-19, COVID-19 vaccines should be celebrated with, by and for all, countering the division on COVID-19 and mutated strains, encompassing tests for COVID-19, positive messages give hope, even in a COVID-19 world, how safe, effective, durable, and affordable are warp speed COVID-19 vaccines?, correlations of severe COVID-19 viral responses, known and unknown long haul effects from COVID-19, how should approved COVID-19 vaccines be distributed globally?, anaphylactic reactions to Pfizer's COVID-19 vaccine, open access is excellent, but who should pay, especially during COVID-19?, fast spreading COVID-19 mutations, the reality of COVID19 in Africa, how does COVID-19 affect cancer and its treatment in Africa?, duration of immune memory to COVID-19, COVID-19 mutations and vaccinations, COVID-19 lineage dynamics, mutations, and vaccines, COVID-19, immunity, and endemicity, and T cells, immune systems, aging hosts in a COVID-19 world, social robots assisting humans in the COVID-19 era, herd immunity against COVID-19 through infection or vaccination, cross-reactive immunity to zoonotic diseases like COVID-19, and multivalent nanobodies, neutralization, suppression of COVID-19 escape mutants, public debate about science is essential in a COVID-19 world, scientific reporting on COVID-19 in China, circadian rhythms and social jet lag in COVID-19, COVID-19 social control measures should have been implemented years ago, tackling student mental healthcare, especially during COVID-19, modelling prioritized COVID-19 vaccine strategies, optimal vaccination strategies for COVID-19, COVID-19 spillovers between species, comovements in science and policy during COVID-19, mathematical and statistical models to guide informed COVID-19 responses, preclinical efficacy and targeting of SARS-CoV-2 and COVID-19, engineering potent antibodies against SARS-CoV-2 and COVID-19, viral mutants escaping antibodies for treating COVID-19, can China help to alleviate bilateral debt payments during COVID-19?, pricing allowances to mitigate carbon dioxide emissions during COVID-19, sequencing SARS-CoV-2 RNA genomes to mitigate COVID-19, unanswered questions about 
antiviral immunity to COVID-19, neutralization of antibodies against escaped mutants, adapting vaccines to escaped mutants, public health and social measures during COVID-19 vaccinations, interpreting the outcomes of COVID-19 tests, disinfection against COVID-19 and antimicrobial resistance, augmenting antiviral immune responses of vaccines for COVID-19, age demographics propelling higher waves of COVID-19, escaping antibodies, recurrent deletions, evading immunity from SARS-CoV-2 and COVID-19, escaped mutants and herd immunity, new vaccines for mild to severe symptoms from COVID-19, escaped mutants of SARS-CoV-2 and COVID-19, are vaccines safe, effective, and durable against escaped mutants?, ethnic diversity in clinical trials for better COVID-19 vaccines, contact tracing for COVID-19 helps everyone, Science metric initiatives, viral sequencing in detecting COVID-19 strains and variants, single-domain antibodies that neutralize replication of SARS-CoV-2 and COVID-19, tracing SARS-CoV-2 and COVID-19 in the UK, COVID-19 penalty for academic mothers, a balanced life during COVID-19, lipopeptides, intranasal prophylaxis, and SARS-CoV-2 transmission, impaired exhaustion and severe COVID-19, would a universal COVID-19 vaccine be safe, effective, and durable?, main protease of SARS-CoV-2 antivirals inhibit replication, temporal duration from COVID-19 to preCOVID-19 normal, mentoring and supervision are more important than the research topic, especially during COVID-19, can mutations in genes confer antibiotic resistance?, connecting violent entertainment and aggression, vaccines are essential for herd immunity from COVID-19, COVID-19 vaccines should be available to everyone, especially healthcare workers, which COVID-19 vaccines are safe, effective, and durable?, building confidence to face a PhD committee, especially during COVID-19, academic openness and national security during COVID-19, antiviral activity against SARS-CoV-2, ignoring the status of retracted COVID-19 papers is academic fake news, vaccinating children and adolescents against COVID-19, inhibiting SARSCoV-2 by enhancing host cell infection, testing COVID-19 megatrial therapies and treatments, new scientific frontiers in the time of COVID-19, questionable data throw school openings during COVID-19 under a spotlight, supply chains and COVID-19 vaccines: The need for speed, research lessons from COVID-19, transmissibility and impact of the UK COVID-19 lineage, neutralizing the impact of the UK COVID-19 lineage, within-host diversity and transmission of COVID-19, partial immunity from COVID-19 vaccine dosing regimes, great challenges need great ideas, especially for COVID-19, hoarding COVID-19 vaccines is unethical, COVID-19 vaccination passports for international travel, naming COVID-19 strains, variants, and lineages, zoom 
meetings for mothers during COVID-19, research is supposed to be enjoyable, COVID-19 or no COVID-19, asymptomatic and presymptomatic COVID-19 cases, impaired immunity through evolving SARS-CoV-2 variants, how many mentors is optimal, especially during COVID-19?, why is trust in COVID-19 vaccines volatile?, the science behind the AstraZeneca COVID-19 vaccine, practical carbon capture in a COVID-19 world, redesigning vaccines to be effective against COVID-19 variants, is it sensible to restrict AstraZeneca by age?, protecting scientists from COVID-19 burnout, adding technology to the NSF for scientific competitiveness, causality between COVID-19 cases and vaccination, expanding the narrative behind the development of COVID-19 vaccines, and confirmed cases, deaths, and vaccinations against COVID-19 in Brazil.

The diverse topics, which include the effectiveness of vaccines against mutations, and establishing trust is hard work, distrust is easy, transmission of COVID-19 between mink and humans, the war against COVID-19 deniers, SARS-CoV-2 is already endemic, COVID-19 efficacy, safety is essential for any vaccine for SARS-CoV-2, aerosol transmission of SARS-CoV2, pre-existing immunity to COVID-19, de novo mutations, and frequency and duration of COVID-19 reinfection, SARS-CoV-2 transmission between mink and humans, further known unknowns about T cell immunity to COVID-19, modeling confusion to clarity in a COVID-19 world, determining serological correlates of COVID-19 severity, what came first, effective COVID-19 vaccines or trial ethics?, safety et al. requirements for COVID-19 vaccines, controlling transmission heterogeneities and controllability of SARS-CoV-2 and COVID-19, and transmission dynamics for a seemingly never ending COVID-19 pandemic, susceptibility to and long hauler effects from severe COVID-19, attack rates of COVID-19 in the Brazilian Amazon, humoral immunity to severe COVID-19, phylogenetic analysis of COVID-19 superspreading events, seasonal flu versus seasonal COVID-19, controlling the spread of COVID-19, antibody responses to severe COVID-19, direct and indirect COVID-19 effects on the heart, government interventions against COVID-19, COVID-19 vaccines should be celebrated with, by and for all, countering the division on COVID-19 and mutated strains, encompassing tests for COVID-19, positive messages give hope, even in a COVID-19 world, and how safe, effective, durable, and affordable are warp speed COVID-19 vaccines?, correlations of severe COVID-19 viral responses, known and unknown long haul effects from COVID-19, how should approved COVID-19 vaccines be distributed globally?, anaphylactic reactions to Pfizer's COVID-19 vaccine, and open access is excellent, but who should pay, especially during COVID-19?, fast spreading COVID-19 mutations, 
the reality of COVID-19 in Africa, how does COVID-19 affect cancer and its treatment in Africa?, duration of immune memory to COVID-19, COVID-19 mutations and vaccinations, COVID-19 lineage dynamics, mutations, and vaccines, COVID-19, immunity, and endemicity, and T cells, immune systems, iging hosts in a COVID-19 world, social robots assisting humans in the COVID19 era, herd Immunity against COVID-19 through infection or vaccination, cross-reactive immunity to zoonotic diseases like COVID-19, and multivalent nanobodies, neutralization, suppression of COVID-19 escape mutants, public debate about science is essential in a COVID19 world, scientific reporting on COVID-19 in China, circadian rhythms and social jet lag in COVID-19, COVID-19 social control measures should have been implemented years ago, tackling student mental healthcare, especially during COVID-19, modelling prioritized COVID-19 vaccine strategies, optimal vaccination strategies for COVID-19, COVID-19 spillovers between species, comovements in science and policy during COVID-19, mathematical and statistical models to guide informed COVID-19 responses, preclinical efficacy and targeting of SARS-CoV-2 and COVID-19, engineering potent antibodies against SARS-CoV-2 and COVID-19, viral mutants escaping antibodies for treating COVID-19, can China help to alleviate bilateral debt payments during COVID-19?, pricing allowances to mitigate carbon dioxide emissions during COVID-19, sequencing SARS-CoV-2 RNA genomes to mitigate COVID-19, unanswered questions about antiviral immunity to COVID-19, neutralization of antibodies against escaped mutants, adapting vaccines to escaped mutants, public health and social measures during COVID-19 vaccinations, interpreting the outcomes of COVID-19 tests, disinfection against COVID-19 and antimicrobial resistance, augmenting antiviral immune responses of vaccines for COVID-19, age demographics propelling higher waves of COVID-19, escaping antibodies, recurrent deletions, evading immunity from SARS-CoV-2 and COVID-19, escaped mutants and herd immunity, new vaccines for mild to severe symptoms from COVID-19, escaped mutants of SARS-CoV-2 and COVID-19, are vaccines safe, effective, and durable against escaped mutants?, ethnic diversity in clinical trials for better COVID-19 vaccines, contact tracing for COVID-19 helps everyone, Science metric initiatives, viral sequencing in detecting COVID-19 strains and variants, single-domain antibodies that neutralize replication of SARS-CoV-2 and COVID-19, tracing SARS-CoV-2 and COVID-19 in the UK, COVID-19 penalty for academic mothers, a balanced life during COVID-19, lipopeptides, intranasal prophylaxis, and SARS-CoV-2 transmission, impaired exhaustion and severe COVID-19, would a universal COVID-19 vaccine be safe, effective, and durable?, main 
protease of SARS-CoV-2 antivirals inhibit replication, temporal duration from COVID-19 to preCOVID-19 normal, mentoring and supervision are more important than the research topic, especially during COVID-19, can mutations in genes confer antibiotic resistance?, connecting violent entertainment and aggression, vaccines are essential for herd immunity from COVID-19, COVID-19 vaccines should be available to everyone, especially healthcare workers, which COVID-19 vaccines are safe, effective, and durable?, building confidence to face a PhD committee, especially during COVID-19, academic openness and national security during COVID-19, antiviral activity against SARS-CoV-2, ignoring the status of retracted COVID-19 papers is academic fake news, vaccinating children and adolescents against COVID-19, inhibiting SARSCoV-2 by enhancing host cell infection, testing COVID-19 megatrial therapies and treatments, new scientific frontiers in the time of COVID-19, questionable data throw school openings during COVID-19 under a spotlight, supply chains and COVID-19 vaccines: The need for speed, research lessons from COVID-19, transmissibility and impact of the UK COVID-19 lineage, neutralizing the impact of the UK COVID-19 lineage, within-host diversity and transmission of COVID-19, partial immunity from COVID-19 vaccine dosing regimes, great challenges need great ideas, especially for COVID-19, hoarding COVID-19 vaccines is unethical, COVID-19 vaccination passports for international travel, naming COVID-19 strains, variants, and lineages, zoom meetings for mothers during COVID-19, research is supposed to be enjoyable, COVID-19 or no COVID-19, asymptomatic and presymptomatic COVID-19 cases, impaired immunity through evolving SARS-CoV-2 variants, how many mentors is optimal, especially during COVID-19?, why is trust in COVID-19 vaccines volatile?, the science behind the AstraZeneca COVID-19 vaccine, practical carbon capture in a COVID-19 world, redesigning vaccines to be effective against COVID-19 variants, is it sensible to restrict AstraZeneca by age?, protecting scientists from COVID-19 burnout, adding technology to the NSF for and expanding the narrative behind the development of COVID-19 vaccines scientific competitiveness, causality between COVID-19 cases and vaccination, and expanding the narrative behind the development of COVID-19 vaccines, and confirmed cases, deaths, and vaccinations against COVID-19 in Brazil, demonstrate that there are still many unanswered questions, or known unknowns, regarding the impact of COVID-19 on the international community.

As COVID-19 is exploding on a daily basis throughout several waves, the number of total cases continues to expand exponentially rather than flattening. As numerous mutations of the 
original wild-type coronavirus are being detected globally, including mutated strains, variants, and lineages of the original wild-type coronavirus, aka COVID-19, eternal vigilance is essential as the increasingly higher waves, now determined at three or four in some continents, show no evidence of slowing down. 


\section{References}

Addai, B.W. and W. Ngwa (2021), COVID-19 and cancer in Africa, Science, published online 1 January 2021, 371(6524), pp.25-27, DOI: 10.1126/science.abd1016, eLetter published online 2 January 2021 (WHO COVID-19 Global literature on coronavirus disease: covidwho-1004079). https://science.sciencemag.org/content/371/6524/25

Beck, D.B. and I. Aksentijevich (2020), Susceptibility to severe COVID-19, Science, published online 23 October 2020, 370(6515), pp.404-405, DOI: 10.1126/science.abe7591, eLetter published online 8 December 2020 (WHO COVID-19 Global literature on coronavirus disease: covidwho-889837).

https://science.sciencemag.org/content/370/6515/404

Bharti, N. (2020), Controlling the coronavirus narrative, Science, published online 14 August 2020, 369(6505), pp.780, DOI: 10.1126/science.abd3662, eLetter published online 16 November 2020 (WHO COVID-19 Global literature on coronavirus disease: covidwho-725925).

https://science.sciencemag.org/content/369/6505/780.2

Billi, A.C. (2021), In aged hosts, T cells turn traitorous, Science Translational Medicine, published online 13 January 2021, 13(576), eabg1759, DOI: 10.1126/scitranslmed.abg1759.

https://stm.sciencemag.org/content/13/576/eabg1759

Bouveret, R. (2020), A bearer of good news, Science, published online 18 December 2020, 370(6523), pp.1522, DOI: 10.1126/science370.6523.1522, eLetter published online 23 December 2020.

https://science.sciencemag.org/content/370/6523/1522

Brainard, J. (2021), Open access takes flight, Science, published online 1 January 2021, 371(6524), pp. 16-20, DOI: 10.1126/science.371.6524.16, Letter published online 2 January 2021.

https://science.sciencemag.org/content/371/6524/16

Brauner, J.M., S. Mindermann, M. Sharma, D. Johnston, J. Salvatier et al. (2021), Inferring the effectiveness of government interventions against COVID-19, Science, published online 19 February 2021, 371(6531), eabd9338, DOI: 10.1126/science.abd9338, eLetter published online 16 December 2020 (WHO COVID-19 Global literature on coronavirus disease: covidwho-978764). https://science.sciencemag.org/content/early/2020/12/15/science.abd9338

Bubar, K.M., K. Reinholt, S.M. Kissler, M. Lipsitch, S. Cobey, Y.H. Grad and B. Larremore (2021), Model-informed COVID-19 vaccine prioritization strategies by age and serostatus, Science, published online 26 February 2021, 371(6532), pp.916-921, DOI: 10.1126/science.abe6959, eLetter published online 22 January 2021 (WHO COVID-19 Global literature on coronavirus disease: covidwho-1041328). 
Bumpus, N.N. (2021), For better drugs, diversify clinical trials, Science, published online 5 February 2021, 371(6529), pp. 570-571, DOI: 10.1126/science.abe2565, eLetter published online 11 February 2021.

https://science.sciencemag.org/content/371/6529/570

Buss, L.F., C.A. Prete Jr, C.M.M. Abrahim, A. Mendrone Jr, T. Salomon et al. (2020), Threequarters attack rate of SARS-CoV-2 in the Brazilian Amazon during a largely unmitigated epidemic, Science, published online 8 December 2020, eabe9728, DOI: 10.1126/science.abe9728, eLetter published online 8 December 2020 (WHO COVID-19 Global literature on coronavirus disease: covidwho-965798).

https://science.sciencemag.org/content/early/2020/12/07/science.abe9728.full

Casassus, B. (2021), France turns to citizens' panel to reduce vaccine skepticism, Science, published online 19 February 2021, 371(6531), pp. 763-764, DOI: 10.1126/science.371.6531.763, eLetter published online 23 February 2021.

https://science.sciencemag.org/content/371/6531/763

Castillo, J.C., A. Ahuja, S. Athey, A. Baker, E. Budish, T. Chipty, R. Glennerster et al. (2021), Market design to accelerate COVID-19 vaccine supply, Science, published online 25 February 2021, eabg0889, DOI: 10.1126/science.abg0889, eLetter published online 11 March 2021 (WHO COVID-19 Global literature on coronavirus disease: covidwho-1105398).

https://science.sciencemag.org/content/371/6534/1107.full

Chandrashekhar, V. (2021), India speeds up vaccinations as cases soar again, Science, published online 2 April 2021, 372(6537), pp. 12-13, DOI: 10.1126/science.372.6537.12.

https://science.sciencemag.org/content/372/6537/12

Cohen, J. (2020a), Shots of hope, Science, published online 18 December 2020, 370(6523), pp. 1392-1394, DOI: 10.1126/science.370.6523.1392, eLetter published online 22 December 2020. https://science.sciencemag.org/content/370/6523/1392

Cohen, J. (2020b), Amid the cheering, some vaccines face questions, Science, published online 4 December 2020, 370(6521), pp. 1151, DOI: 10.1126/science.370.6521.1151, eLetter published online 9 February 2021.

https://science.sciencemag.org/content/370/6521/1151.full

Cohen, J. (2021a), Two new vaccines deliver good and bad news for the pandemic, Science, published online 5 February 2021, 371(6529), pp. 548-549, DOI: 10.1126/science.371.6529.548, eLetter published online 8 February 2021.

https://science.sciencemag.org/content/371/6529/548

Cohen, J. (2021b), The long road, Science, published online 19 February 2021, 371(6531), pp. 768-772, DOI: 10.1126/science.371.6531.768, eLetter published online 19 February 2021. https://science.sciencemag.org/content/371/6531/768 
Cohen, J. (2021c), Dosing debates, transparency issues roil vaccine rollouts, Science, published online 8 January 2021, 371(6525), pp. 109-110, DOI: 10.1126/science.371.6525.109, eLetter published online 23 February 2021.

https://science.sciencemag.org/content/371/6525/109

Cohen, J. and K. Kupferschmidt (2020), As vaccines emerge, a global waiting game begins, Science, published online 18 December 2020, 370(6523), pp. 1385-1387, DOI: 10.1126/science.370.6523.1385, eLetter published online 30 December 2020 (WHO COVID-19 Global literature on coronavirus disease: covidwho-1008343).

https://science.sciencemag.org/content/370/6523/1385.full

Cohen, J. and K. Kupferschmidt (2021), As vaccine surpluses loom, donation plans urged, Science, published online 12 March 2021, 371(6534), pp. 1087-1088, DOI: 10.1126/science.371.6534.1087, eLetter published online 18 March 2021.

https://science.sciencemag.org/content/371/6534/1087.full

Cohen, A.A., P.N.P. Gnanapragasam, Y.E. Lee, P.R. Hoffman, S. Ou, M. Kakutani et al. (2021), Mosaic nanoparticles elicit cross-reactive immune responses to zoonotic coronaviruses in mice, Science, published online 12 February 2021, 371(6530), pp. 735-741, DOI: 10.1126/science.abf6840, eLetter published online 15 January 2021.

https://science.sciencemag.org/content/371/6530/735.full

Collins, F.S. (2021), COVID-19 lessons from research, Science, published online 12 March 2021, 371(6534), pp. 1081, DOI: 10.1126/science.abh3996, eLetter published online 11 March 2021. https://science.sciencemag.org/content/371/6534/1081.full

Conn, R.W. (2021), Expanding the endless frontier, Science, published online 2 April 2021, 372(6537), pp. 9, DOI: 10.1126/science.abi7486.

https://science.sciencemag.org/content/372/6537/9.full

Couzin-Frankel, J. (2020), The long haul, Science, published online 7 August 2020, 369(6504), pp. 614-617, DOI: 10.1126/science.369.6504.614, eLetter published online 30 December 2020. https://science.sciencemag.org/content/369/6504/614.full

Couzin-Frankel, J. (2021), Vaccine trials ramp up in children and adolescents, Science, published online 26 February 2021, 371(6532), pp. 874-875, DOI: 10.1126/science.371.6532.874, eLetter published online 1 March 2021.

https://science.sciencemag.org/content/371/6532/874

Dalchand, N. (2021), A perfect illusion, Science, published online 26 February 2021, 371(6532), pp. 958, DOI: 10.1126/science.379.6532.958, eLetter published online 23 February 2021. https://science.sciencemag.org/content/371/6532/958

Dan, J.M., J. Mateus, Y. Kato, K.M. Hastie, E.D. Yu, C.E. Faliti et al. (2021), Immunological memory to SARS-CoV-2 assessed for up to 8 months after infection, Science, published online 5 
February 2021, 371(6529), eabf4063, DOI: 10.1126/science.abf4063, eLetter published online 6 January 2021 (WHO COVID-19 Global literature on coronavirus disease: covidwho-934059). https://science.sciencemag.org/content/371/6529/eabf4063.full

Davies, N.G., S. Abbott, R.C. Barnard, C.I. Jarvis, A.D. Kucharski et al. (2021), Estimated transmissibility and impact of SARS-CoV-2 lineage B.1.1.7 in England, Science, published online 3 March 2021, eabg3055, DOI: 10.1126/science.abg3055, eLetter published online 12 March 2021 (WHO COVID-19 Global literature on coronavirus disease: covidwho-1117640). https://science.sciencemag.org/content/early/2021/03/03/science.abg3055.full

de Vries, R.D., K.S. Schmitz, F.T. Bovier, C. Predella, J. Khao et al. (2021), Intranasal fusion inhibitory lipopeptide prevents direct-contact SARS-CoV-2 transmission in ferrets, Science, published online 17 February 2021, eabf4896, DOI: 10.1126/science.abf4896, eLetter published online 17 February (WHO COVID-19 Global literature on coronavirus disease: covidwho1088184). https://science.sciencemag.org/content/early/2021/02/16/science.abf4896

de Vrieze, J. (2020), Reinfections, still rare, provide clues on immunity, Science, published online 20 November 2020, 370(6519), pp. 895-897, DOI: 10.1126/science.370.6519.895, eLetter published online 20 November 2020.

https://science.sciencemag.org/content/370/6519/895

de Vrieze, J. (2021), Pfizer's vaccine raises allergy concerns, Science, published online 1 January 2021, 371(6524), pp. 10-11, DOI: 10.1126/science.371.6524.10, eLetter published online 31 December 2020.

https://science.sciencemag.org/content/371/6524/10

du Plessis, L., J.T. McCrone, A.E. Zarebski, V. Hill, C. Ruis et al. (2021), Establishment and lineage dynamics of the SARS-CoV-2 epidemic in the UK, Science, published online 12 February 2021, 371(6530), pp. 708-712, DOI: 10.1126/science.abf2946, eLetter published online 12 January 2021 (WHO COVID-19 Global literature on coronavirus disease: covidwho-1015589). https://science.sciencemag.org/content/371/6530/708.full

Dye, C. and M.C. Mills (2021), COVID-19 vaccination passports, Science, published online 19 March 2021, 371(6535), pp. 1184, DOI: 10.1126/science.abi5245, eLetter published online 19 March 2021.

https://science.sciencemag.org/content/371/6535/1184.full

Fauci, A.S. (2021), The story behind COVID-19 vaccines, Science, published online 9 April 2021, 372(6538), pp. 109, DOI: 10.1126/science.abi8397.

https://science.sciencemag.org/content/372/6538/109.full

Fanous, H., A. Guerrero-Pena and A. Pillai (2020), B careful: Humoral responses and COVID-19 severity, Science Immunology, 5(54), eabf8870, published online 4 December 2020, DOI: 10.1126/sciimmunol.abf8870, eLetter published online 9 December 2020 (WHO COVID-19 Global literature on coronavirus disease: covidwho-961006). 
https://immunology.sciencemag.org/content/5/54/eabf8870.full

Faria, N.R., T.A. Mellan, C. Whittaker, I.M. Claro, D. da S. Candido, S. Mishra et al. (2021), Genomics and epidemiology of the P.1 SARS-CoV-2 lineage in Manaus, Brazil, Science, published online 14 April 2021, eabh2644, DOI: 10.1126/science.abh2644

https://science.sciencemag.org/content/early/2021/04/13/science.abh2644

Fitzpatrick, M.C. and A.P. Galvani (2021), Optimizing age-specific vaccination, Science, published online 26 February 2021, 371(6532), pp. 890-891, DOI: 10.1126/science.abg2334, eLetter published online 22 January 2021.

https://science.sciencemag.org/content/371/6532/890.full

Fujiwara, R. (2021), Balancing act, Science, published online 12 February 2021, 371(6530), pp. 750, DOI: 10.1126/science.371.6530.750, eLetter published online 12 February 2021. https://science.sciencemag.org/content/371/6530/750.full

Galanti, M., S. Pei, T.K. Yamana, F.J. Angulo, A. Charos, D.L. Swerdlow and J. Shaman (2021), Social distancing remains key during vaccinations, Science, published online 29 January 2021, 371(6528), pp. 473-474, DOI: 10.1126/science.abg2326, eLetter published online 2 February 2021. https://science.sciencemag.org/content/371/6528/473.2

Guthmiller, J.J. and P.C. Wilson (2020), Remembering seasonal coronaviruses, Science, published online 11 December 2020, 370(6522), pp. 1272-1273, DOI: 10.1126/science.abf4860, eLetter published online 12 December 2020 (WHO COVID-19 Global literature on coronavirus disease: covidwho-970457).

https://science.sciencemag.org/content/370/6522/1272.full

Haynes, B.F., L. Corey, P. Fernandes, P.B. Gilbert, P.J. Hotez, S. Rao et al. (2020), Prospects for a safe COVID-19 vaccine, Science Translational Medicine, published online 4 November 2020, 12(568), eabe0948, DOI: 10.1126/scitranslmed.abe0948, eLetter published online 5 December 2020 (WHO COVID-19 Global literature on coronavirus disease: covidwho-880947).

https://stm.sciencemag.org/content/12/568/eabe0948

Hope, J.L. and L.M. Bradley (2021), Lessons in antiviral immunity, Science, published online 29 January 2021, 368(6493), pp. 860-868, DOI: 10.1126/science.abb5793, eLetter published online 29 January 2021.

https://science.sciencemag.org/content/371/6528/464/tab-e-letters

Hou, Y.J., S. Chiba, P. Halfmann, C. Ehre, M. Kuroda, K.H. Dinnon III et al. (2020), SARS-CoV2 D614G variant exhibits efficient replication ex vivo and transmission in vivo, Science, published online 12 November 2020, eabe8499, DOI: 10.1126/science.abe8499, eLetter published online 14 November 2020 (WHO COVID-19 Global literature on coronavirus disease: covidwho-922513). https://science.sciencemag.org/content/early/2020/11/11/science.abe8499 
Hurley, J.M. (2021), Could COVID-19 eliminate the alarm clock?, Science Translational Medicine, published online 20 January 2021, 13(577), eabg4724, DOI: 10.1126/scitranslmed.abg4723. https://stm.sciencemag.org/content/13/577/eabg4723

Jayaweera, M., H. Perera, B. Gunawardana and J. Manatunge (2020), Transmission of COVID-19 virus by droplets and aerosols: A critical review on the unresolved dichotomy, Environmental Research, 88, September 2020, published online 2020 June 13, doi: 10.1016/j.envres.2020.109819 (WHO COVID-19 Global literature on coronavirus disease: covidwho-597359). https://www.sciencedirect.com/science/article/pii/S0013935120307143?via\%3Dihub

Juno, J.A. and S.L. O'Connor (2021), Translating viral vaccines into immunity, Science, published online 29 January 2021, 371(6528), pp. 460-461, DOI: 10.1126/science.abf8121, eLetter published 3 February 2021 (WHO COVID-19 Global literature on coronavirus disease: covidwho1054605).

https://science.sciencemag.org/content/371/6528/460

Karim, S.A.A., T. de Oliveira and G. Loots (2021), Appropriate names for COVID-19 variants, Science, published online 19 March 2021, 371(6535), pp. 1215, DOI: 10.1126/science.abh0836. eLetter published online 19 March 2021.

https://science.sciencemag.org/content/371/6535/1215

Karlsson, A.C., M. Humbert and M. Buggert (2020), The known unknowns of T cell immunity to COVID-19, Science Immunology, 5(53), eabe8063, published online 18 November 2020, DOI: 10.1126/sciimmunol.abe8063, eLetter published online 26 November 2020 (WHO COVID-19 Global literature on coronavirus disease: covidwho-934059).

https://immunology.sciencemag.org/content/5/53/eabe8063

Kielian, M. (2020), Enhancing host cell infection by SARS-CoV-2, Science, published online 12 November 2020,370(6518), pp. 765-766, DOI: 10.1126/science.abf0732, eLetter published online 1 March 2021 (WHO COVID-19 Global literature on coronavirus disease: covidwho-922514 and covidwho-1004089).

https://science.sciencemag.org/content/370/6518/765

Kissler, S.M., C. Tedijanto, E. Goldstein, Y.H. Grad and M. Lipsitch (2020), Projecting the transmission dynamics of SARS-CoV-2 through the postpandemic period, Science, published online 22 May 2020, 368(6493), pp. 860-868, DOI: 10.1126/science.abb5793, eLetter published online 8 December 2020 (WHO COVID-19 Global literature on coronavirus disease: covidwho57045).

https://science.sciencemag.org/content/368/6493/860/tab-e-letters

Klompas, M., M.A. Baker and C. Rhee (2020), Airborne transmission of SARS-CoV-2 Theoretical considerations and available evidence, Journal of the American Medical Association (JAMA), published online 13 July 2020, doi:10.1001/jama.2020.12458 (WHO COVID-19 Global literature on coronavirus disease: covidwho-641580). https://jamanetwork.com/journals/jama/fullarticle/2768396 
Knipe, D.M., O. Levy, K.A. Fitzgerald and A. Mühlberger (2020), Ensuring vaccine safety, Science, published online 17 November 2020, eabf0357, DOI: 10.1126/science.abf0357, eLetter published online 17 November 2020.

https://science.sciencemag.org/content/early/2020/11/16/science.abf035721

Koenig, P.-A., H. Das, H. Liu, B.M. Kümmerer, F.N. Gohr, L.-M. Jenster et al. (2021), Structureguided multivalent nanobodies block SARS-CoV-2 infection and suppress mutational escape, Science, published online 12 February 2021, 371(6530), eabe6230, DOI: 10.1126/science.abe6230, eLetter published online 15 January 2021. https://science.sciencemag.org/content/371/6530/eabe6230.full

Koff, W.C. and S.F. Berkley (2021), A universal coronavirus vaccine, Science, published online 19 February 2021, 371(6531), pp. 759, DOI: 10.1126/science.abh0447, eLetter published online 19 February 2021. https://science.sciencemag.org/content/371/6531/759

Kupferschmidt, K. (2020), A divisive disease, Science, published online 18 December 2020, 370(6523), pp. 1395-1397, DOI: 10.1126/science.370.6523.1395, eLetter published online 13 December 2020.

https://science.sciencemag.org/content/370/6523/1395.full

Kupferschmidt, K. (2021a), Fast-spreading U.K. virus variant raises alarms, Science, published online 1 January 2021, 371(6524), pp. 9-10, DOI: 10.1126/science.371.6524.9, eLetter published online 1 January 2021 (WHO COVID-19 Global literature on coronavirus disease: covidwho1048636).

https://science.sciencemag.org/content/371/6524/9/tab-e-letters

Kupferschmidt, K. (2021b), Viral evolution may herald new pandemic phase, Science, published online 8 January 2021, 371(6525), pp. 108-109, DOI: 10.1126/science.371.6525.108, eLetter published online 10 January 2021 (WHO COVID-19 Global literature on coronavirus disease: covidwho-1042790).

https://science.sciencemag.org/content/371/6525/108.full

Kupferschmidt, K. (2021c), Vaccinemakers ponder how to adapt to virus variants, Science, published online 29 January 2021, 371(6528), pp. 448-449, DOI: 10.1126/science.371.6528.448, eLetter published online 2 February 2021.

https://science.sciencemag.org/content/371/6528/448.full

Kupferschmidt, K. (2021d), New mutations raise specter of 'immune escape', Science, published online 22 January 2021, 371(6527), pp. 329-330, DOI: 10.1126/science.371.6527.329, eLetter published online 8 February 2021 (WHO COVID-19 Global literature on coronavirus disease: covidwho-1063044).

https://science.sciencemag.org/content/371/6527/329 
Kupferschmidt, K. (2021e), Danish scientists see tough times ahead as variant rises, Science, published online 5 February 2021, 371(6529), pp. 549-550, DOI: 10.1126/science.371.6529.549, eLetter published online 8 February 2021.

https://science.sciencemag.org/content/371/6529/549

Kupferschmidt, K. (2021f), Deaths of health workers in Africa highlight vaccine inequity, Science, published online 19 February 2021, 371(6531), pp. 764-765, DOI: 10.1126/science.371.6531.764, eLetter published online 23 February 2021.

https://science.sciencemag.org/content/371/6531/764

Kupferschmidt, K. (2021g), WHO's treatment megatrial is at a standstill, Science, published online 5 March 2021, 371(6533), pp. 972-973, DOI: 10.1126/science.371.6533.972, eLetter published online 4 March 2021.

https://science.sciencemag.org/content/371/6533/972.full

Kusnadi, A., C. Ramírez-Suástegui, V. Fajardo, S.J. Chee, B.J. Meckiff et al. (2021), Severely ill COVID-19 patients display impaired exhaustion features in SARS-CoV-2-reactive CD8 ${ }^{+} \mathrm{T}$ cells, Science Immunology, 6(55), eabe4782, published online 21 January 2021, DOI: 10.1126/sciimmunol.abe4782 (WHO COVID-19 Global literature on coronavirus disease: covidwho-1042797).

https://immunology.sciencemag.org/content/6/55/eabe4782

Langin, K. (2021), Pandemic hit academic mothers hard, data show, Science, published online 12 February 2021, 371(6530), pp. 660, DOI: 10.1126/science.371.6530.660, eLetter published online 12 February 2021.

https://science.sciencemag.org/content/371/6530/660.full

Larsen, M.D., E.L. de Graaf, M.E. Sonneveld, R. Plomp, J. Nouta, W. Hoepel et al. (2021), Afucosylated IgG characterizes enveloped viral responses and correlates with COVID-19 severity, Science, published online 26 February 2021, 37(6532), eabc8378, DOI: 10.1126/science.abc8378, eLetter published online 25 December 2020 (WHO COVID-19 Global literature on coronavirus disease: covidwho-999185).

https://science.sciencemag.org/content/371/6532/eabc8378.full

Larson, H.J. and D.A. Broniatowski (2021), Volatility of vaccine confidence, Science, published online 26 March 2021, 371(6536), pp. 1289, DOI: 10.1126/science.abi6488.

https://science.sciencemag.org/content/371/6536/1289

Lavine, J.S., O.N. Bjornstad and R. Antia (2021), Immunological characteristics govern the transition of COVID-19 to endemicity, Science, published online 12 February 2021, 371(6530), pp. 741-745, DOI: 10.1126/science.abe6522, eLetter published online 13 January 2021 (WHO COVID-19 Global literature on coronavirus disease: covidwho-1029163).

https://science.sciencemag.org/content/371/6530/741.full

Lee, E.C., N.I. Wada, M.K. Grabowski, E.S. Gurley and J. Lessler (2020), The engines of SARSCoV-2 spread, Science, published online 23 October 2020, 370(6515), pp. 406-407, DOI: 
10.1126/science.abd87555, eLetter published online 13 December 2020 (WHO COVID-19 Global literature on coronavirus disease: covidwho-889833, covidwho-975635).

https://science.sciencemag.org/content/370/6515/406.full

Lemieux, J.E., K.J. Siddle, B.M. Shaw, C. Loreth, S.F. Schaffner et al. (2020), Phylogenetic analysis of SARS-CoV-2 in Boston highlights the impact of superspreading events, Science, published online 10 December 2020, eabe3261, DOI: 10.1126/science.abe3261, eLetter published online 11 December 2020 (WHO COVID-19 Global literature on coronavirus disease: ppmedrxiv20178236).

https://science.sciencemag.org/content/early/2020/12/09/science.abe3261.full

Leshner, A.I. (2021), Target student mental well-being, Science, published online 22 January 2021, 371(6527), pp. 325, DOI: 10.1126/science.abg5770.

https://science.sciencemag.org/content/371/6527/325

Lesté-Lasserre, C. (2020), Pandemic dooms Danish mink - and mink research, Science, published online 13 November 2020, 370(6518), pp. 754, DOI: 10.1126/science.370.6518.754, eLetters published online 15 November 2020; 24 November 2020 (WHO COVID-19 Global literature on coronavirus disease: 973183 ).

https://science.sciencemag.org/content/370/6518/754

Lipsitch, M. and N.E. Dean (2020), Understanding COVID-19 vaccine efficacy, Science, published online 13 November 2020, 370(6518), pp. 763-765, DOI: 10.1126/science.abe5938, eLetter published online 17 November 2020 (WHO COVID-19 Global literature on coronavirus disease: covidwho-889836).

https://science.sciencemag.org/content/370/6518/763

Lodish, H., J. Chen and P. Sharp (2021), Clarity on the crackdown, Science, published online 26 February 2021, 371(6532), pp. 867, DOI: 10.1126/science.abh1627, eLetter published online 23 February 2021.

https://science.sciencemag.org/content/371/6532/867

Lopatkin, A.J., S.C. Bening, A.L. Manson, J.M. Stokes, M.A. Kohanski et al. (2021), Clinically relevant mutations in core metabolic genes confer antibiotic resistance, Science, published online 19 February 2021, 371(6531), eaba0862, DOI: 10.1126/science.aba0862, eLetter published online 19 February 2021.

https://science.sciencemag.org/content/371/6531/eaba0862

Lu, J. and J. Guo (2021), Disinfection spreads antimicrobial resistance, Science, published online 29 January 2021, 371(6528), pp. 474, DOI: 10.1126/science.abg4380, eLetter published 3 February 2021.

https://science.sciencemag.org/content/371/6528/474.1

Luchsinger, L.L. and C.D. Hillyer (2021), Vaccine efficacy probable against COVID-19 variants, Science, published online 12 March 2021, 371(6534), pp. 1116, DOI: 10.1126/science.abg9461 (WHO COVID-19 Global literature on coronavirus disease: covidwho-1133121). 
https://science.sciencemag.org/content/371/6534/1116.1.full

Lythgoe, K.A., M. Hall, L. Ferretti, M. de Cesare, G. MacIntyre-Cockett et al. (2021), SARSCoV-2 within-host diversity and transmission, Science, published online 9 March 2021, eabg0822, DOI: 10.1126/science.abg0821, eLetter published online 12 March 2021 (WHO COVID-19 Global literature on coronavirus disease: covidwho-1125076).

https://science.sciencemag.org/content/early/2021/03/10/science.abg0821.full

Maeda, J.M. and J.N. Nkengasong (2021), The puzzle of the COVID-19 pandemic in Africa, Science, published online 1 January 2021, 371(6524), pp. 27-28, DOI: 10.1126/science.abf8832, eLetter published online 2 January 2021 (WHO COVID-19 Global literature on coronavirus disease: covidwho-1004091).

https://science.sciencemag.org/content/371/6524/27/tab-e-letters

Martin, M.A., D. VanInsberghe and K. Koelle (2021), Insights from SARS-CoV-2 sequences, Science, published online 29 January 2021, 371(6524), pp. 27-28, DOI: 10.1126/science.abf8832, eLetter published online 29 January 2021 (WHO COVID-19 Global literature on coronavirus disease: covidwho-1054602).

https://science.sciencemag.org/content/371/6528/466/tab-e-letters

McCarthy, K.R., L.J. Rennick, S. Nambulli, R. Robinson-McCarthy, W.G. Bain et al. (2021), Recurrent deletions in the SARS-CoV-2 spike glycoprotein drive antibody escape, Science, published online 3 February 2021, eabf6950, DOI: 10.1126/science.abf6950, eLetter published online 5 February 2021 (WHO COVID-19 Global literature on coronavirus disease: covidwho1063045).

https://science.sciencemag.org/content/early/2021/02/02/science.abf6950

McCormick, K.D., J.L. Jacobs and J.W. Mellors (2021), The emerging plasticity of SARS-CoV2, Science, published online 26 March 2021, 371(6536), pp. 1306-1308, DOI: 10.1126/science.abg4493.

https://science.sciencemag.org/content/371/6536/1306.full

Mertz, A.F. and A. Mishra (2021), Science's new frontier, Science, published online 5 March 2021, 371(6533), pp. 967, DOI: 10.1126/science.abh3185, eLetter published online 5 March 2021. https://science.sciencemag.org/content/371/6533/967.full

Metcalf, C.J.E., D.H. Morris and S.W. Park (2020), Mathematical models to guide pandemic response, Science, published online 24 July 2020, 369(6502), pp. 368-369, DOI: 10.1126/science.abd1668, eLetter published online 16 January 2021. https://science.sciencemag.org/content/369/6502/368/tab-e-letters

Mina, M.J. and K.G. Andersen (2020), COVID-19 testing: One size does not fit all, Science, published online 21 December 2020, eabe9187, DOI: 10.1126/science.abe9187, eLetter published 21 December 2020.

https://science.sciencemag.org/content/early/2020/12/18/science.abe9187 
Monod, M., A. Blenkinsop, X. Xi, D. Hebert, S. Bershan, S. Tietze et al. (2021), Age groups that sustain resurging COVID-19 epidemics in the United States, Science, published online 2 February 2021, eabe8372, DOI: 10.1126/science.abe8372, eLetter published 4 February 2021 (WHO COVID-19 Global literature on coronavirus disease: covidwho-1061088). https://science.sciencemag.org/content/early/2021/02/01/science.abe8372

Moore, E. (2021), The more mentors, the merrier, Science, published online 26 March 2021, 371(6536), pp. 1398, DOI: 10.1126/science.371.6536.1398. https://science.sciencemag.org/content/371/6536/1398.full

Muik, A., A.-K. Wallisch, B. Sänger, K.A. Swanson, J. Mühl, W. Chen, H. Cai et al. (2021), Neutralization of SARS-CoV-2 lineage B.1.1.7 pseudovirus by BNT162b2 vaccine-elicited human sera, Science, published online 12 March 2021, 371(6534), pp. 1152-1153, DOI: 10.1126/science.abg6105, eLetters published online 2 February 2021, 12 March 2021 (WHO COVID-19 Global literature on coronavirus disease: covidwho-1054606).

https://science.sciencemag.org/content/371/6534/1152

Naseer, N. and K. Strelau (2021), Tracing contacts, finding ourselves, Science, published online 5 February 2021, 371(6529), pp. 646, DOI: 10.1126/science.371.6529.646, eLetter published online 11 February 2021.

https://science.sciencemag.org/content/371/6529/646

Nelson, M.I. (2021), Tracking the UK SARS-CoV-2 outbreak, Science, published online 12 February 2021, 371(6530), pp. 680-681, DOI: 10.1126/science.abg2297, eLetter published online 12 February 2021 (WHO COVID-19 Global literature on coronavirus disease: covidwho1083130).

https://science.sciencemag.org/content/371/6530/680.full

Ng, K.W., N. Faulkner, G.H. Cornish, A. Rose, R. Harvey, S. Hussain et al. (2020), Preexisting and de novo humoral immunity to SARS-CoV-2 in humans, Science, published online 6 November 2020, eabe1107, DOI: 10.1126/science.abe1107, eLetter published online 19 November 2020 (WHO COVID-19 Global literature on coronavirus disease: covidwho-913669). https://science.sciencemag.org/content/early/2020/11/05/science.abe1107

Normile, D. (2021), Pandemic could mark 'turning point' for Chinese science, Science, published online 15 January 2021, 371(6526), pp. 222-223, DOI: 10.1126/science.371.6526.222, eLetter published online 18 January 2021.

https://science.sciencemag.org/content/371/6526/222.full

O'Grady, C. (2021), Media and aggression research retracted after scrutiny, Science, published online 19 February 2021, 371(6531), pp.767, DOI: 10.1126/science.371.6531.767, eLetter published online 22 February 2021.

https://science.sciencemag.org/content/371/6531/767

Oude Munnink, B.B., R.S. Sikkema, D.F. Nieuwenhuijse, R.J. Molenaar, E. Munder et al. (2020), Transmission of SARS-CoV-2 on mink farms between humans and mink and back to humans, Science, published online 10 November 2020, eabe1107, DOI: 10.1126/science.abe1107, eLetter 
published online 26 November 2020 (WHO COVID-19 Global literature on coronavirus disease: covidwho-919190).

https://science.sciencemag.org/content/early/2020/11/09/science.abe5901/tab-e-letters

Piller, C. (2021), Disgraced COVID-19 studies are still routinely cited, Science, published online 22 January 2021, 371(6527), pp. 331-332, DOI: 10.1126/science.371/6527.331 (WHO COVID19 Global literature on coronavirus disease: covidwho-1043675).

https://science.sciencemag.org/content/371/6527/331

Prather, K.A., L.C. Marr, R.T. Schooley, M.A. McDiarmid, M.E. Wilson and D.K. Milton (2020), Airborne transmission of SARS-CoV-2, Science, published online 16 October 2020, 370(6514), pp. 303-304, DOI: 10.1126/science.abf0521, eLetter published online 19 November 2020 (WHO COVID-19 Global literature on coronavirus disease: covidwho-814250).

https://science.sciencemag.org/content/370/6514/303.2

Press, W.H. and R.C. Levin (2020), Modeling, post COVID-19, Science, published online 27 November 2020, 370(6520), p. 1015, DOI: 10.1126/science.abf7914, eLetter published online 17 November 2020 (WHO COVID-19 Global literature on coronavirus disease: covidwho-947800). https://science.sciencemag.org/content/370/6520/1015

Qiao, J., Y.-S. Li, R. Zeng, F.-L. Liu, R.-H. Luo, C. Huang et al. (2021), SARS-CoV-2 $\mathrm{M}^{\text {pro }}$ inhibitors with antiviral activity in a transgenic mouse model, Science, published online 18 February 2021, eabf1611, DOI: 10.1126/science.abf1611, eLetter published online 19 February 2021 (WHO COVID-19 Global literature on coronavirus disease: covidwho-covidwho-1091003). https://science.sciencemag.org/content/early/2021/02/17/science.abf1611

Rappazzo, C.G., L.V. Tse, C.I. Kaku, D. Wrapp, M. Sakharkar, D. Huang et al. (2021), Broad and potent activity against SARS-like viruses by an engineered human monoclonal antibody, Science, published online 19 February 2021, 371(6531), pp. 823-829, DOI: 10.1126/science.abf4830, eLetter published online 27 January 2021.

https://science.sciencemag.org/content/early/2021/01/22/science.abf4830

Rasmussen, A.L. and S.V. Popescu (2021), SARS-CoV-2 transmission without symptoms, Science, published online 19 March 2021, 371(6535), pp. 1206-1207, DOI: 10.1126/science.abf9569, eLetter published online 24 March 2021.

https://science.sciencemag.org/content/371/6535/1206.full

Robinson-McCarthy, L.R., A.J. Mijalis, G.T. Filsinger, H. de Puig, N.M. Donghia, T.E. Schaus et al. (2021), Anomalous COVID-19 tests hinder researchers, Science, published online 15 January 2021, 371(6526), pp. 224-245, DOI: 10.1126/science.abf8873, eLetter published online 3 February 2021 (WHO COVID-19 Global literature on coronavirus disease: covidwho-1032128). https://science.sciencemag.org/content/371/6526/244

Röltgen, K., A.E. Powell, O.F. Wirz, B.A. Stevens, C.A. Hogan et al. (2020), Defining the features and duration of antibody responses to SARS-CoV-2 infection associated with disease severity and outcome, Science Immunology, 5(54), eabe0240, published online 7 December 2020, DOI: 
10.1126/sciimmunol.abe8063, eLetter published online 22 December 2020 (WHO COVID-19 Global literature on coronavirus disease: covidwho-963892).

https://immunology.sciencemag.org/content/5/54/eabe0240/tab-e-letters

Saad-Roy, C.M., S.E. Morris, C.J.E. Metcalf, M.J. Mina, R.E. Baker et al. (2021), Epidemiological and evolutionary considerations of SARS-CoV-2 vaccine dosing regimes, Science, published online 9 March 2021, eabg8663, DOI: 10.1126/science.abg8663, eLetter published online 12 March 2021 (WHO COVID-19 Global literature on coronavirus disease: covidwho-1125155). https://science.sciencemag.org/content/early/2021/03/08/science.abg8663.full

Saelens, X. and B. Schepens (2021), Single-domain antibodies make a difference, Science, published online 12 February 2021, 371(6530), pp. 681-682, DOI: 10.1126/science.abg2294, eLetter published online 12 February 2021.

https://science.sciencemag.org/content/371/6530/681.full

Scassellati, B. and M. Vázquez (2020), The potential of socially assistive robots during infectious disease outbreaks, Science Robotics, 5(44), eabc9014, published online 15 July 2020, DOI: 10.1126/scirobotics.abc9014, eLetter published 14 January 2021.

https://robotics.sciencemag.org/content/5/44/eabc9014.full

Service, R.F. (2021a), A call to arms, Science, published online 12 March 2021, 371(6534), pp. 1092-1095, DOI: 10.1126/science.371.6534.1092, eLetter published online 18 March 2021. https://science.sciencemag.org/content/371/6534/1092/tab-e-letters

Service, R.F. (2021b), Carbon capture marches toward practical use, Science, published online 26 March 2021, 371(6536), pp. 1300, DOI: 10.1126/science.371.6536.1300.

https://science.sciencemag.org/content/371/6536/1300

Servick, K. (2021), COVID-19 measures also suppress flu - for now, Science, published online 15 January 2021, 371(6526), pp. 224, DOI: 10.1126/science.371.6526.224, eLetter published online 22 January 2021 (WHO COVID-19 Global literature on coronavirus disease: covidwho-1032151). https://science.sciencemag.org/content/371/6526/224.full

Shaman, J. and M. Galanti (2020), Will SARS-CoV-2 become endemic?, Science, published online 30 October 2020, 370(6516), pp. 527-529, DOI: 10.1126/science.abe5960, eLetter published online 17 November 2020 (WHO COVID-19 Global literature on coronavirus disease: covidwho-873449).

https://science.sciencemag.org/content/370/6516/527

Shen, M.J. (2021), A mother's plea, Science, published online 12 March 2021, 371(6534), pp. 1174, DOI: 10.1126/science.371.6534.1174, eLetter published online 19 March 2021. https://science.sciencemag.org/content/371/6534/1174

Shrock, E., E. Fujimura, T. Kula, R.T. Timms, I.-H. Lee, Y. Leng et al. (2020), Viral epitope profiling of COVID-19 patients reveals cross-reactivity and correlates of severity, Science, published online 27 November 2020, 370(6520), DOI: 10.1126/science.abd4250, eLetter 
published online 29 November 2020 (WHO COVID-19 Global literature on coronavirus disease: covidwho-809284).

https://science.sciencemag.org/content/370/6520/eabd4250

Simmons, B.A., R. Ray, H. Yang and K.P. Gallagher (2021), China can help solve the debt and environmental crises, Science, published online 29 January 2021, 371(6528), pp. 468-470, DOI: 10.1126/science.abf4049, eLetter published online 29 January 2021 (WHO COVID-19 Global literature on coronavirus disease: covidwho-1054603).

https://science.sciencemag.org/content/371/6528/468/tab-e-letters

Sridhar, D. and D. Gurdasani (2021), Herd immunity by infection is not an option, Science, published online 15 January 2021, 371(6526), pp. 230-231, DOI: 10.1126/science.abf7921, eLetter published online 14 January 2021.

https://science.sciencemag.org/content/371/6526/230

Starr, T.N., A.J. Greaney, A. Addetia, W.W. Hannon, M.C. Choudhary et al. (2021), Prospective mapping of viral mutations that escape antibodies used to treat COVID-19, Science, published online 19 February 2021, 371(6531), pp. 850-854, DOI: 10.1126/science.abf9302, eLetter published online 27 January 2021 (WHO COVID-19 Global literature on coronavirus disease: covidwho-1048645).

https://science.sciencemag.org/content/early/2021/01/22/science.abf9302

Sun, K., W. Wang, L. Gao, Y. Wang, K. Luo, L. Ren, Z. Zhan, X. Chen et al. (2020), Transmission heterogeneities, kinetics, and controllability of SARS-CoV-2, Science, published online 24 November 2020, eabe2424, DOI: 10.1126/science.abe2424, eLetter published online 5 December 2020 (WHO COVID-19 Global literature on coronavirus disease: covidwho-944842).

https://science.sciencemag.org/content/early/2020/11/23/science.abe2424

Thorp, H.H. (2020), A breakthrough for us all, Science, published online 18 December 2020, 370(6523), pp. 1381, DOI: 10.1126/science.abg1254, eLetter published online 17 December 2020. https://science.sciencemag.org/content/370/6523/1381

Thorp, H.H. (2021a), Public debate is good for science, Science, published online 15 January 2021, 371(6526), pp. 213, DOI: 10.1126/science.abg4685, eLetter published online 16 January 2021. https://science.sciencemag.org/content/371/6526/213

Thorp, H.H. (2021b), Monitoring change, Science, published online 12 February 2021, 371(6530), pp. 653, DOI: 10.1126/science.abg9540, eLetter published online 12 February 2021. https://science.sciencemag.org/content/371/6530/653.full

Topol, E.J. (2020), COVID-19 can affect the heart, Science, published online 23 October 2020, 370(6515), pp. 408-409, DOI: 10.1126/science.abe2813, eLetter published online 17 December 2020 (WHO COVID-19 Global literature on coronavirus disease: covidwho-793688). https://science.sciencemag.org/content/370/6515/408 
Tuckson, R.V. (2020), The disease of distrust, Science, published online 13 November 2020, 370(6518), pp. 745, DOI: 10.1126/science.abf6109, eLetter published online 15 November 2020 (WHO COVID-19 Global literature on coronavirus disease: covidwho-926210).

https://science.sciencemag.org/content/370/6518/745

Vogel, G. (2021), Data in paper about Swedish schoolchildren come under fire, Science, published online 5 March 2021, 371(6533), pp. 973-974, DOI: 10.1126/science.371.6533.973, eLetter published online 9 March 2021.

https://science.sciencemag.org/content/371/6533/973

Vogel, G. and K. Kupferschmidt (2021a), New problems erode confidence in AstraZeneca's vaccine, Science, published online 26 March 2021, 371(6536), pp. 1294-1295, DOI: 10.1126/science.371.6536.1294, eLetter published online 26 March 2021.

https://science.sciencemag.org/content/371/6536/1294.full

Vogel, G. and K. Kupferschmidt (2021b), Side effect worry grows for AstraZeneca vaccine, Science, published online 2 April 2021, 372(6537), pp. 14-15, DOI: 10.1126/science.372.6537.14. https://science.sciencemag.org/content/372/6537/14

Voosen, P. (2021), Trump downplayed the cost of carbon. That's about to change, Science, published online 29 January 2021, 371(6528), pp. 447-448, DOI: 10.1126/science.371.6528.447, eLetter published online 29 January 2021.

https://science.sciencemag.org/content/371/6528/447/tab-e-letters

Wadman, M. (2021a), United States rushes to fill void in viral sequencing, Science, published online 12 February 2021, 371(6530), pp. 657-658, DOI: 10.1126/science.371.6530.657, eLetter published online 12 February 2021.

https://science.sciencemag.org/content/371/6530/657.full

Wadman, M. (2021b), Pandemic scientist fight burnout, Science, published online 2 April 2021, 32 (6537), pp. 13-14, DOI: 10.1126/science.372.6537.13.

https://science.sciencemag.org/content/372/6537/13.full

Wendler, D., J. Ochoa, J. Millum, C. Grady and H.A. Taylor (2020), COVID-19 vaccine trial ethics once we have efficacious vaccines, Science, published online 3 December 2020, eabf5084, DOI: 10.1126/science.abf5084, eLetter published online 4 December 2020 (WHO COVID-19 Global literature on coronavirus disease: covidwho-961005).

https://science.sciencemag.org/content/early/2020/12/02/science.abf5084

Wengert, E. (2021), Mentor as you'd want to be mentored, Science, published online 19 February 2021, 371(6531), pp. 858, DOI: 10.1126/science.371.6531.858, eLetter published online 19 February 2021.

https://science.sciencemag.org/content/371/6531/858

White, K.M., R. Rosales, S. Yildiz, T. Kehrer, L. Miorin, E. Moreno et al. (2021), Plitidepsin has potent preclinical efficacy against SARS-CoV-2 by targeting the host protein eEF1A, Science, 
published online 26 February 2021, 371(6532), pp. 926-931, DOI: 10.1126/science.abf4058, eLetter published 26 January 2021 (WHO COVID-19 Global literature on coronavirus disease: covidwho-1048642).

https://science.sciencemag.org/content/371/6532/926.full

Wong, J.P. and B. Damania (2021), SARS-CoV-2 dependence on host pathways, Science, published online 26 February 2021, 371(6532), pp. 884-885, DOI: 10.1126/science.abg6837, eLetter published online 26 February 2021 (WHO COVID-19 Global literature on coronavirus disease: covidwho-1105401).

https://science.sciencemag.org/content/371/6532/884.full

World Health Organization (2020), WHO COVID-19 Global literature on coronavirus disease, https://www.who.int/emergencies/diseases/novel-coronavirus-2019/global-research-on-novelcoronavirus-2019-ncov, accessed 18 November 2020.

Wright, T. (2021), What sparks joy, Science, published online 19 March 2021, 371(6535), pp. 1278, DOI: 10.1126/science.371.6535.1278, eLetter published online 19 March 2021. https://science.sciencemag.org/content/371/6535/1278

Yin, Y., J. Gao, B.F. Jones and D. Wang (2021), Coevolution of policy and science during the pandemic, Science, published online 8 January 2021, 371(6525), pp. 120-130, DOI: 10.1126/science.abe3084, eLetter published online 8 January 2021 (WHO COVID-19 Global literature on coronavirus disease: covidwho-1015586). https://science.sciencemag.org/content/371/6525/128/tab-e-letters

Zeng, W.B., X.L. Wang, J.Y. Li et al. (2020), Association of daily wear of eyeglasses with susceptibility to coronavirus disease 2019 infection, Journal of the American Medical Association (JAMA) Ophthalmology, doi:10.1001/jamaophthalmol.2020.3906, published online 16 September 2020 (WHO COVID-19 Global literature on coronavirus disease: covidwho-762984). https://jamanetwork.com/journals/jamaophthalmology/fullarticle/2770872

Zhou, P. and Z.-L. Shi (2021), SARS-CoV-2 spillover events, Science, published online 8 January 2021, 371(6525), pp. 120-122, DOI: 10.1126/science.abf6097, eLetter published online 8 January 2021 (WHO COVID-19 Global literature on coronavirus disease: covidwho-961005). https://science.sciencemag.org/content/371/6525/120/tab-e-letters 